\title{
ON 2-GROUPS WITH NO NORMAL ABELIAN SUBGROUPS OF RANK 3, AND THEIR OCCURRENCE AS SYLOW 2-SUBGROUPS OF FINITE SIMPLE GROUPS
}

\author{
BY \\ ANNE R. MACWILLIAMS( $\left.{ }^{1}\right)$
}

\begin{abstract}
We prove that in a finite 2-group with no normal Abelian subgroup of rank $\geqq 3$, every subgroup can be generated by four elements. This result is then used to determine which 2-groups $T$ with no normal Abelian subgroup of rank $\geqq 3$ can occur as $S_{2}$ 's of finite simple groups $G$, under certain assumptions on the embedding of $T$ in $G$.
\end{abstract}

Introduction. In view of the Feit-Thompson theorem (namely, that all finite groups of odd order are solvable), it is natural to classify and search for finite simple groups by considering the structure of a Sylow 2-subgroup and its embedding in the group.

There are comparatively few 2-groups with no normal Abelian subgroup of rank 2 -such a 2-group must be cyclic, dihedral, semidihedral, or generalized quaternion. Of these, only dihedral and semidihedral groups can (and do) occur as Sylow 2subgroups of simple groups (Brauer [4, Theorem 2, p. 321]). However, there is a greater range of 2-groups with no normal Abelian subgroup of rank 3; this paper is part of a project to determine which such 2-groups can occur as Sylow 2-subgroups of simple groups.

The first result is a theorem about 2-groups with no normal Abelian subgroup of rank 3 (apart from questions about simple groups). We show that every subgroup of such a 2-group can be generated by four elements. This is best-possible; in fact, there are 2-groups satisfying the hypothesis and having elementary subgroups of rank 4. Analogs of the four-generator theorem for odd primes are more restrictive; for instance, if $p$ is odd and $P$ is a $p$-group with no normal Abelian subgroup of rank 3 , then every subgroup of $P$ can be generated by three elements (Thompson; Huppert [9, Satz III. 12.3, p. 343]) and every Abelian subgroup of $P$ can be generated by two elements (Feit and Thompson [6, Lemma 8.4(i), p. 797]).

Received by the editors September 12, 1969.

AMS Subject Clasisifications. Primary 2029, 2040; Secondary 2025, 2027, 2043.

Key Words and Phrases. p-group, Sylow p-subgroup, automorphism, rank, generators, conjugate, transfer homomorphism, fusion.

( $\left.{ }^{1}\right)$ The author thanks the National Science Foundation for its support, via a National Science Foundation Graduate Fellowship, during this work.

Copyright (C) 1970, American Mathematical Society 
We then suppose that $T$ is a 2-group with a normal Abelian subgroup of rank 2 but none of rank $3 ; T \not D_{8}$; and $T$ is a Sylow 2-subgroup of a simple group $G$. It then follows (Thompson [10]) that $T$ has exactly one normal four-group, say $W$. We work out all the possibilities for $T$, using constructive methods together with fusion arguments, under each of the following additional assumptions:

1. $\left[N_{G}(T): C_{G}(T)\right]$ is divisible by odd primes (so that $T$ admits an automorphism of odd order).

2. $\left[N_{G}(T): C_{G}(T)\right]$ is a power of 2 , and the three involutions of $W$ are all fused together in $G$. (Then $C_{T}(W)$ is a maximal subgroup of $T$ and admits an automorphism of order 3.)

If we assume $\left[N_{G}(T): C_{G}(T)\right]$ is divisible by odd primes (Theorem 1), we find that 3 divides $\left[N_{G}(T): C_{G}(T)\right.$ ], and: If $N_{G}(T) / C_{G}(T)$ contains a subgroup of order 3 which centralizes $W$, then $T$ is isomorphic to the Sylow 2-subgroup of the JankoHall and Janko-Higman groups; also $G$ has either one or two conjugacy classes of involutions, and if two, it is determined which fusions of $T$-classes of involutions take place in $G$. (The Janko-Hall group has two classes of involutions and the Janko-Higman group has one.) If $N_{G}(T) / C_{G}(T)$ contains a subgroup of order 3 which does not centralize $W$, then $T$ is either a four-group or is isomorphic to the Sylow 2-subgroup of $\mathrm{PSU}_{3}$ (16).

If we assume $\left[N_{G}(T): C_{G}(T)\right]$ is a power of 2 and the involutions of $W$ are all $G$-conjugate (Theorem 2), we find that: Either $T$ is isomorphic to the Sylow 2subgroup of $\operatorname{PSL}_{3}(q)$ for $q \equiv 1 \bmod 4$ (that is, $T \cong Z_{2} r \nmid Z_{2}$ for $r \geqq 2$ ); or $T=\left\langle Z_{2} r \nmid Z_{2}, z\right\rangle$ where $z$ either inverts the base group or raises it to the power $-1+2^{r-1}$; or $T$ is a certain group of order $2^{8}$.

Finally we prove what may be regarded as a sort of converse to Theorem 1; namely, that any simple group $G$ with a Sylow 2-subgroup $T$ isomorphic to the Sylow 2-subgroup of the Janko-Hall and Janko-Higman groups, must have $N_{G}(T): C_{G}(T)$ divisible by odd primes.

Notations. Let $X$ be a group. We write $Z(X)$ for the center of $X$, and $\Phi(X)$ for the Frattini subgroup of $X$; and Aut $(X)$, Inn $(X)$ for the groups of automorphisms, respectively inner automorphisms, of $X$. SCN $(X)$ means the set of self-centralizing normal Abelian subgroups of $X ; \operatorname{SCN}_{n}(X)=\{A \in \operatorname{SCN}(X): \operatorname{rank} A \geqq n\}$.

If $Y$ is any subset of $X$, and $f$ is any function on $X$, we write $\left.f\right|_{Y}$ for the restriction of $f$ to $Y$.

If $X_{1}, \ldots, X_{n}$ are subgroups of $X$ with $X=X_{1} \geqq X_{2} \geqq \cdots \geqq X_{n}$ and $X_{i+1}$ normal in $X_{i}$ for $i=1, \ldots, n-1$, the stability group of the chain $X_{1} \geqq \cdots \geqq X_{n}$ means $\left\{\alpha \in \operatorname{Aut}(X): X_{i}^{\alpha}=X_{i}\right.$ for $i=1, \ldots, n$, and $\alpha$ acts trivially on $X_{i} / X_{i+1}$ for $i=1, \ldots, n-1\}$.

An involution of $X$ is an element of order 2 in $X$.

If $x, h \in X$, we write $[h, x]$ for $h^{-1} x^{-1} h x$, and $h^{x}$ for $x^{-1} h x . x$ and $y \in X$ are said to be fused in $X$ if there is $g \in X$ with $x^{g}=y$.

If $X$ and $H$ are subgroups of some group, we write 


$$
\begin{aligned}
N_{X}(H) & =\left\{x \in X: H^{x}=H\right\}, \\
C_{X}(H) & =\left\{x \in X: h^{x}=h \quad \text { for every } h \in H\right\}, \\
C_{X}(h) & =\left\{x \in X: h^{x}=h\right\} \quad \text { for } h \in H .
\end{aligned}
$$

$A_{X}(H)$ means the group of automorphisms of $H$ induced by conjugation from $X$; thus $A_{X}(H) \cong N_{X}(H) / C_{X}(H) . H \triangleleft X$ means $H$ is a normal subgroup (not necessarily proper) of $X$.

If $X$ and $Y$ are groups then $X \hookrightarrow Y$ means $X$ is isomorphic to a subgroup of $Y$; for instance, if $H \leqq G$ are groups, then $N_{G}(H) / C_{G}(H \hookrightarrow$ Aut $(H))$.

If $G$ is a $p$-group, then $\Omega_{n}(G)$ means the subgroup of $G$ generated by the elements of order $\leqq p^{n} ; \mho^{n}(G)$ means the subgroup generated by the $p^{n}$ th powers of the elements of $G$.

$\Sigma_{n}$ respectively $\Sigma_{n}^{+}$denote the symmetric and alternating groups of degree $n$.

$E_{2^{r}}$ denotes an elementary Abelian group of order $2^{r}$.

\section{A theorem on 2-groups with no normal Abelian subgroups of rank 3.}

\subsection{Usefyt'standard results.}

LEMma FA. Let $P$ be a p-group, and let $C, D$ be normal subgroups of $P$ with $C \subseteq D$ and $C$ Abelian. Then there is $K \in \mathrm{SCN}(D)$ with $C \leqq K$ and $K \triangleleft P$.

Proof. Take a subgroup $K$ of $P$ such that (i) $C \leqq K \leqq D, K \triangleleft P$, and $K$ Abelian. (ii) $K$ is maximal subject to (i). Then $C_{D}(K) \triangleleft P$. If $C_{D}(K)>K$, there is a subgroup $R$ of $D$ with $C_{D}(K) \geqq R>K,[R: K]=p$, and $R \triangleleft P$. $R$ is Abelian since $R$ contains centrally a subgroup of index $p$. But then $R$ satisfies (i), contradicting the maximality of $K$.

LEMMA FB. If $G$ is a 2-group, then $\Phi(G)=\left\langle g^{2}: g \in G\right\rangle$.

Proof. In general, the Frattini subgroup of a p-group is generated by its squares and commutators. If $x, y \in G$, then

$$
1 \equiv(x y)^{2}=x y x y=x^{2} x^{-1} y^{2} y^{-1} x y \equiv[x, y] \bmod \left\langle g^{2}: g \in G\right\rangle .
$$

Therefore $[G, G] \subseteq\left\langle g^{2}: g \in G\right\rangle$, and the result follows.

LEMMA FC. If $G$ is a 2-group and $T, N$ are subgroups of $G$ such that $T$ normalizes $N$, then $\Phi(T N)=\langle\Phi(T), \Phi(N),[T, N]\rangle$.

Proof. For $t \in T, n \in N$,

$$
(t n)^{2}=t n t n \cdot=t^{2} t^{-1} n^{2} n^{-1} t n \equiv[t, n] \bmod \langle\Phi(T), \Phi(N)\rangle .
$$

The lemma follows from Lemma FB.

For the next lemma we need to observe a few things about the characters of finite Abelian groups. If $G$ is a finite Abelian group, the irreducible complex characters of $G$ are simply homomorphisms from $G$ to the multiplicative group of the complex field. If $\lambda$ is such a homomorphism, and $g \in G$, we may write the value 
of $\lambda$ at $g$ as $\langle g, \lambda\rangle$. The set of such homomorphisms becomes an Abelian group, denoted by $G^{*}$, if we define the product of $\lambda$ and $\mu$ by $\langle g, \lambda \mu\rangle=\langle g, \lambda\rangle\langle g, \mu\rangle$.

(i) $G^{*} \cong G$.

Proof. Write $G=G_{1} \times \cdots \times G_{k}$ as a direct product of cyclic groups. Then for $\lambda \in G^{*}$, the restriction $\lambda_{i}$ of $\lambda$ to $G_{i}$ is an element of $G_{i}^{*}$. If $\lambda_{i}=1$ for each $i$, then $\lambda=1$. So the map $\eta: \lambda \rightarrow\left(\lambda_{1}, \ldots, \lambda_{k}\right)$ is an injection of $G^{*}$ into $G_{1}^{*} \times \cdots \times G_{k}^{*}$.

However, $\left|G^{*}\right|=$ number of conjugacy classes of $G,=|G|$; and for each $i$, $\left|G_{i}^{*}\right|=\left|G_{i}\right|$, hence $\left|G_{1}^{*} \times \cdots \times G_{k}^{*}\right|=\left|G_{1}^{*}\right| \times \cdots \times\left|G_{k}^{*}\right|=\left|G_{1}\right| \times \cdots \times\left|G_{k}\right|=|G|$. So $\eta$ is an isomorphism of $G^{*}$ onto $G_{1}^{*} \times \cdots \times G_{k}^{*}$. So it will suffice to show that $G_{i}^{*} \cong G_{i}$ for each $i$, i.e. to show that $G^{*} \cong G$ if $G$ is cyclic of order $n$ say.

Let $\omega$ be a primitive complex $n$th root of 1 , and let $G=\langle g\rangle$. Then $\langle g, \lambda\rangle=\omega$ determines an element of $G^{*} .\left\langle g, \lambda^{2}\right\rangle=\omega^{2}$ and so $\lambda, \lambda^{2}, \ldots, \lambda^{n}=1$ are distinct elements of $G^{*}$. Moreover, any element of $G^{*}$ must send $g$ to some $n$th root of 1 ; so $\lambda, \lambda^{2}, \ldots, \lambda^{n}$ exhaust $G^{*}$. So $G^{*}$ is cyclic of order $n$, as desired.

(ii) If $H \leqq G$ for finite Abelian groups $H$ and $G$, then each linear character of $H$ can be extended to one of $G$.

Proof. Let $\lambda \in H^{*}$ and let $\mu$ be an irreducible constituent of $\lambda^{G}=$ the character of $G$ induced by $\lambda$. $\left(\lambda^{G}, \mu\right)=\left(\lambda,\left.\mu\right|_{H}\right)$ by Frobenius reciprocity. So $\lambda$ is a constituent of $\left.\mu\right|_{H}$. But $\lambda$ and $\mu$ are both linear, hence $\lambda=\left.\mu\right|_{H}$; i.e., $\mu$ extends $\lambda$.

(iii) If $G$ is a finite Abelian group, then the correspondences

$$
\begin{gathered}
\qquad N \stackrel{\varphi}{\longrightarrow}\left\{\lambda \in G^{*}: \operatorname{ker} \lambda \supseteq N\right\} \\
\text { (Intersection of the kernels of the } \lambda \in A) \stackrel{\theta}{\longleftarrow} A
\end{gathered}
$$

between subgroups of $G$ and $G^{*}$, invert one another, so define a one-one correspondence. Moreover, if $N \leftrightarrow A$, then $N \cong G^{*} / A$.

Proof. The pairing $\left\langle,>\right.$ between the two Abelian groups $G$ and $G^{*}$ treats $G$ and $G^{*}$ symmetrically; more precisely, $G$ plays the same role to $G^{*}$ with respect to $\langle$,$\rangle as does G^{*}$ to $G$. The correspondences $\varphi$ and $\theta$ can be rephrased:

$$
\begin{aligned}
& N \stackrel{\varphi}{\longrightarrow}\left\{\lambda \in G^{*}:\langle g, \lambda\rangle=1 \text { for all } g \in N\right\} \\
& \{g \in G:\langle g, \lambda\rangle=1 \text { for all } \lambda \in A\} \stackrel{\theta}{\longleftarrow} A .
\end{aligned}
$$

Thus it is clear that $\varphi$ and $\theta$ are abstractly the same. So if we show $\theta \varphi=1$ (i.e., for every subgroup $N$ of $G, \theta(\varphi(N))=N)$, we will also have shown $\varphi \theta=1$.

We now show $\theta \varphi=1$. Let $M=\theta(\varphi(N)) ; M \supseteq N$. If $M>N$, then $M / N$ has a nontrivial linear character $\lambda$. By (ii), there is a linear character $\mu$ of $G / N$ whose restriction to $M / N$ is $\lambda$. We may view $\mu$ as a character of $G$, with $N \subseteq \operatorname{ker} \mu$. Then $\mu \in \varphi(N)$; but $M \nsubseteq \operatorname{ker} \mu$, so $M \nsubseteq \theta(\varphi(N))$.

Given a subgroup $N$ of $G$, let $A=\varphi(N)$. Two elements of $G^{*}$ are congruent $\bmod A$ if and only if their restrictions to $N$ agree; so $G^{*} /\left.A \cong G^{*}\right|_{N}$. By (ii), $\left.G^{*}\right|_{N}=N^{*}$. Also, by (i), $N^{*} \cong N$; so $N \cong G^{*} / A$, as claimed. 
Now let $G$ be an arbitrary finite group. The set of linear characters of $G$ acts on the set of all characters of $G$, by multiplication: for $\lambda$ linear and $\chi$ arbitrary, $\chi \rightarrow \chi \lambda$.

For $\chi$ an arbitrary character of $G$, define

$$
V(\chi)=\frac{1}{|G|} \sum_{g \in G} \chi\left(g^{2}\right)
$$

Then (Feit [5, Vol. 1, p. 24]) $\sum_{x} V(\chi) \chi(1)$ (where $\chi$ runs over all the irreducible characters of $G$ ) is the number of elements of $G$ whose square is 1 .

(iv) If $\lambda$ is linear and $\lambda^{2}=1$, then $V(\chi \lambda)=V(\chi)$.

Proof. $V(\chi \lambda)=(1 /|G|) \sum_{g} \chi\left(g^{2}\right) \lambda\left(g^{2}\right)$; but $\lambda\left(g^{2}\right)=\lambda(g)^{2}=1$ since $\lambda^{2}=1$.

(v) If $\chi$ is any irreducible character of $G, N$ is a normal subgroup of $G$, and $\chi$ vanishes on $G-N$, then $[G: N] \leqq \chi(1)^{2}$.

Proof. $1=(\chi, \chi)_{G}=(|N| /|G|)\left(\left.\chi\right|_{N},\left.\chi\right|_{N}\right)_{N}$. By Clifford's theorem, $\left.\chi\right|_{N}=a \sum_{i=1}^{t} \theta_{i}$ where the $\theta_{i}$ are $G$-conjugate and so all of the same degree. So

$$
\left(\left.\chi\right|_{N},\left.\chi\right|_{N}\right)_{N}=a^{2} t \leqq a^{2} t^{2} \leqq\left(\operatorname{at}\left(\operatorname{deg} \theta_{i}\right)\right)^{2}=\chi(1)^{2} .
$$

Hence $1 \leqq(|N| /|G|) \chi(1)^{2}$, as desired.

LEMMA FD. If $G$ is a 2-group with $[G: \Phi(G)] \geqq 2^{2 k+1}$, then $\sum_{x} V(\chi) \chi(1)$ $\equiv 0 \bmod 2^{k+1}$ (the sum ranging over all irreducible characters $\chi$ of $G$ ).

Proof. For each $\chi, V(\chi)$ is an integer (Feit [5]); so we need only consider the $\chi$ with degree $\leqq 2^{k}$.

Suppose $\chi(1)=2^{k-e}(e \geqq 0) . A=(G / \Phi(G))^{*}$ acts on the irreducible characters of $G$ by $\lambda: \chi \rightarrow \chi \lambda$. We will show $\chi$ is in an orbit of size $\geqq 2^{e+1}$, and then Lemma FD will follow from (iv).

Let $C$ be the subgroup of $A$ fixing $\chi$; then the size of the orbit containing $\chi$ is [ $A: C$ ]. Let $N$ be the intersection of the kernels of the $\lambda \in C$. Then for $g \notin N$, there is $\lambda \in C$ with $\lambda(g) \neq 1$, but $\lambda(g) \chi(g)=\chi(g)$; hence $\chi(g)=0$ for all $g \notin N$. So by (v), $\chi(1)^{2} \geqq[G: N]$. But $[G: N]=|C|$ by (iii). So

$$
|C| \leqq \chi(1)^{2}=\left(2^{k-e}\right)^{2}=2^{2 k-2 e} ;
$$

hence $[A: C] \geqq 2^{(2 k+1)-(2 k-2 e)}=2^{2 e+1} \geqq 2^{e+1}$, as desired.

1.2. Proof of the theorem.

FOUR GENERATOR THEOREM. Let $G$ be a 2-group with no normal Abelian subgroup of rank 3. Then every subgroup of $G$ can be generated by four (or fewer) elements.

Proof of four generator theorem. Assume false and let $G$ be a counterexample of minimal order.

(i) If $G$ has a subgroup $T$ with $[T: \Phi(T)] \geqq 2^{6}$ (i.e., $T$ requires more than five generators), then $G$ has a subgroup $U$ with $[U: \Phi(U)]=2^{5}$.

Thus to prove the theorem, we need only show $G$ has no subgroups $T$ with - $[T: \Phi(T)]=2^{5}$. 
Proof. Choose $T$ so that $[T: \Phi(T)] \geqq 2^{6}$ and $|T|$ is minimal subject to this condition. There is a subgroup $U$ of $T$ with $[U: \Phi(T)]=2^{5}$. If $\Phi(U)$ is proper in $\Phi(T)$, then $[U: \Phi(U)] \geqq 2^{6}$, which contradicts the minimality of $|T|$.

(ii) If $G$ has a cyclic subgroup $K$ of index $\leqq 2^{3}$, then $G$ has no subgroups $T$ with $[T: \Phi(T)]=2^{5}$.

Proof. For such a $T, 2^{3} \geqq[G: K] \geqq[T: T \cap K] \geqq 2^{4}$, the last inequality because $T / \Phi(T)$ is elementary of rank 5 , and $T \cap K$ is cyclic so can project onto at most a subgroup of order 2 in $T / \Phi(T)$.

(iii) $G$ has a normal four-group, $W$ say.

Proof. If not, $G$ is cyclic or is a 2-group of maximal class (Blackburn [3, Theorem 1.1 , p. 3]). So $G$ contains a cyclic subgroup of index 2 (Blackburn [2, Theorem 3.4, p. 68]). This contradicts (ii) since $G$ is a counterexample to the theorem.

(iv) $W$ is the only normal four-group of $G$.

Proof. Let $X$ be a normal four-group of $G, X \neq W,[X, W] \subseteq X \cap W$. If $X$ centralizes $W$ then $X W$ is a normal Abelian subgroup of order $\geqq 8$, and is elementary since generated by involutions; so $G$ has a normal $E_{8}$, contrary to hypothesis. So $[X, W]=X \cap W$ is of order 2 , and $D=X W \cong D_{8}$.

$G$ stabilizes the two chains $X>X \cap W>1$ and $W>X \cap W>1$, so stabilizes $D>\Phi(D)>1$. The stability group of $D>\Phi(D)>1$ is just $\operatorname{Inn}(D)$; so $G=D C_{G}(D)$. Write $C=C_{G}(D) ;[G: C]=4$.

If $Y$ is a normal four-group of $C$, then $Y \triangleleft G$ and $Y \neq W$ and $Y$ centralizes $W$, so $Y W$ is a normal $E_{8}$ or $E_{16}$ of $G$. So $C$ has no normal four-group. The argument of (iii) (applied to $C$ instead of $G$ ) shows that $C$ has a cyclic subgroup $K$ of index 2; thus $[G: K]=8$, contradicting (ii).

(v) $W \leqq \Phi(G)$.

Proof. If not, let $M$ be a maximal subgroup of $G$ with $W \nsubseteq M$. By Lemma FA (with $C=1$ ), there is $K \in \operatorname{SCN}(M), K \triangleleft G . \Omega_{1}(K) \triangleleft G$ and $\Omega_{1}(K) \neq W$, so $K$ is cyclic by (iv), of order $2^{\xi}$ say. $M / K \hookrightarrow$ Aut $(K)$. $\mid$ Aut $(K) \mid=2^{\xi-1}$ and so $|M| \leqq 2^{\xi} 2^{\xi-1}=2^{2 \xi-1}$.

If $|G|=32$ and $G$ has a five-generator subgroup, $G$ itself is elementary, so $\operatorname{SCN}_{3}(G)$ is nonempty. So $|G| \geqq 64,|M| \geqq 32$. So $2^{5} \leqq 2^{2 \xi-1} ; 5 \leqq 2 \xi-1 ; 3 \leqq \xi$.

We will show $[M: K] \leqq 2$; then $[G: K] \leqq 4$, which contradicts (ii).

Suppose $M>K$. Let $R$ be a subgroup with $M \geqq R>K$ and $[R: K]=2$. $R$ is nonAbelian since $K \in \operatorname{SCN}(M)$. For $\xi \geqq 3$, there are four non-Abelian 2-groups with cyclic subgroups of order $2^{\xi}$ and index 2 ; they are dihedral, semidihedral, generalized quaternion, and $P(\xi)$, where $P(\xi)=\left\langle c, k: k\right.$ of order $2^{\xi}, c$ of order $\left.2, c k c=k^{1+2^{\xi-1}}\right\rangle$. $P(\xi)$ has exactly three involutions, which (together with 1$)$ constitute a characteristic four-group.

Let $R$ be a subgroup with $M \geqq R>K,[R: K]=2$, and $R \triangleleft G$. $R$ either centralizes or inverts $\mho^{1}(K)$ (which is of order $\geqq 4$ ). If $R$ centralizes $\mho^{1}(K)$, then $R \cong P(\xi)$, and its characteristic four-group is normal in $G$ and contained in $M$, so $\neq W$, contrary to (iv). So $R$ inverts $\mho^{1}(K)$. 
Suppose $[M: K] \geqq 4$. Let $S, R$ be subgroups with $M \geqq S>R>K,[S: R]=2$, [R:K] $=2, S, R \triangleleft G$. If $S / K$ is cyclic, then $R / K$ induces on $\mho^{1}(K)$ the square of the automorphism induced by $S / K$. But $R$ inverts $\mho^{1}(K)$ and inversion is not a square in Aut $\left(\mho^{1}(K)\right)$. So $S / K$ is a four-group. Two of the three subgroups properly between $S$ and $K$ invert $\mho^{1}(K)$ and the third centralizes $\mho^{1}(K)$. $G$ permutes these three subgroups, so must normalize the one which centralizes $\mho^{1}(K)$, contrary to the preceding paragraph. So $[M: K] \leqq 2$, as desired.

(vi) Suppose $N$ is a normal subgroup of $G$ which contains $W$ and centralizes $W$. Then $[N: \Phi(N)] \leqq 2^{4}$.

Proof. If $[N: \Phi(N)] \geqq 2^{5}$, then the number of elements of $N$ whose square is 1 is $\equiv 0 \bmod 8$, by Lemma FD. In particular, $N-W$ contains involutions. Since $W$ is central in $N$, the involutions of $N-W$ are partitioned into cosets of $W$; each such coset uniquely determines an $E_{8}$ of $N$ which contains $W$. So the number of such $E_{8}$ 's is $(8 k-4) / 4$ (where $8 k$ is the number of elements of $N$ whose square is 1 ). $(8 k-4) / 4=2 k-1$, an odd number. $N \triangleleft G$, so $G$ permutes these $E_{8}$ 's by conjugation. $G$ is a 2-group, so must normalize one of these $E_{8}$ 's.

(vii) If $W$ is central in $G$, then $|G| \geqq 2^{8}$.

Proof. Since every maximal subgroup of $G$ contains $W, G$ and all its maximal subgroups are four-generator groups by (vi). So if $|G|<2^{8}$, we must have $|G|=2^{7}$ and some subgroup $T$ of index 4 in $G$ is a five-generator group, therefore elementary. Let $M$ be a maximal subgroup of $G$ with $T \leqq M . M=\langle m, T\rangle$ where $m \notin T, m^{2} \in T$. So $m$ induces on $T$ an automorphism $X$ of order 2. Regard $T$ as a vector space over the field of two elements. Then in the ring of linear transformations of $T$, $0=X^{2}-1=(X-1)^{2}$. So

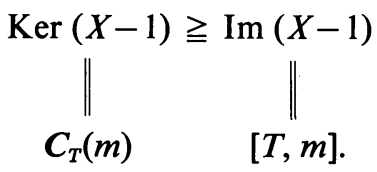

But $\operatorname{Dim}(\operatorname{Ker}(X-1))+\operatorname{Dim}(\operatorname{Im}(X-1))=\operatorname{Dim}(T)=5$. So $\left|C_{T}(m)\right| \geqq 2^{3}$.

$M$ is non-Abelian; for otherwise $\left\langle m^{2}\right\rangle=\Phi(M)$ is of order $\leqq 2$ and so $[M: \Phi(M)] \geqq 2^{5}$, contradicting (vi). Therefore $Z(M)=C_{T}(m) \triangleleft G$, so $G$ has a normal $E_{8}$.

Case I. Some maximal subgroup $M$ of $G$ has $\operatorname{SCN}_{3}(M)$ nonempty. $W \subseteq M$ by (v). $M$ has a normal $E_{8}, E$ say, such that $E \supseteq W$. For if $X$ is any normal $E_{8}$ of $M, X$ acts on $W$ and $\left|C_{x}(W)\right| \geqq 4$; also $C_{x}(W) \triangleleft M$; so if $W \nsubseteq C_{x}(W)$, replace $X$ by a suitable $E_{8}$ of $W C_{x}(W)$.

$E \Varangle G$. Let $H=E^{g}$ for $g \in G-M . E \cap H=W$. Write $V=E H$; then $V \cong D_{8} \times Z_{2}$ and $V \triangleleft G$.

Take $e, h \in G$ with $E=\langle e, W\rangle, H=\langle h, W\rangle$. Write $f=e h$. Then $f^{2}=w$ generates $\Phi(V)$ and so is central in $G . F=\langle f, W\rangle$ is normal in $G$ since it is the only $Z_{4} \times Z_{2}$ of $V$. Take $w_{1} \in W$ such that $W=\left\langle w, w_{1}\right\rangle$. 
Let $C=C_{G}(V) ; C \triangleleft G$. By Lemma FA, there is $B \in \operatorname{SCN}(C)$ with $B \supseteq W$ and $B \triangleleft G$.

(viii) $B$ does not contain $w$ as a square.

Proof. Suppose $x \in B, x^{2}=w$. Then $x \in \Omega_{2}(B) . K=\Omega_{2}(B) F$ is a normal Abelian subgroup of $G$. $K$ contains the three involutions of $W$ and also the involution $x f$. So $\left|\Omega_{1}(K)\right| \geqq 8$ and $\Omega_{1}(K) \triangleleft G$.

Therefore $B=\langle b\rangle \times\langle w\rangle$ for $b$ of order $2^{m}, m \geqq 1$.

Aut $(V) . V$ can be presented as $\left\langle f, e, w_{1}: f^{4}=1, e^{2}=1\right.$, efe $=f^{-1}=f w ; w_{1}^{2}=1$, $w_{1}$ central $\rangle$ where $\langle w\rangle=\Phi(V)$.

Any set of elements $x, y, z$ of $V$ such that (1) $x$ is of order 4 (there are four such $x$ ), (2) $y$ is a noncentral involution of $V$ (there are eight such $y$ ), (3) $z$ is a central involution of $V$ other than $w$ (there are two such $z$ ) will have: $x, y, z$ generate $V$ and satisfy the same relations as those given above for $f, e, w_{1}$. So the automorphisms of $V$ correspond one-to-one with the choices for $x, y, z$. So $\mid$ Aut $(V) \mid=4 \cdot 8 \cdot 2=64$.

Let automorphisms of $V$ be defined as follows:

$$
\begin{aligned}
& \alpha: \quad f \rightarrow f w ; e, w_{1} \rightarrow \text { selves. } \\
& \beta: \quad f, w_{1} \rightarrow \text { selves; } e \rightarrow e w . \\
& \xi: \quad f \rightarrow f w_{1} ; e, w_{1} \rightarrow \text { selves; } h \rightarrow h w_{1} . \\
& \eta: \quad f, w_{1} \rightarrow \text { selves; } e \rightarrow e w_{1} ; h \rightarrow h w_{1} . \\
& \zeta: \quad f, e \rightarrow \text { selves; } w_{1} \rightarrow w w_{1} . \\
& \theta: \quad f, w_{1} \rightarrow \text { selves; } e \rightarrow f e .
\end{aligned}
$$

Then $\alpha$ and $\beta$ are the inner automorphisms induced by $e$ and $f$ respectively. $\langle\alpha, \beta, \xi, \eta\rangle \cong E_{16}$.

$\zeta$ centralizes $\alpha$ and $\beta ; \zeta^{-1} \xi \zeta=\alpha \xi, \zeta^{-1} \eta \zeta=\beta \eta ; \zeta^{2}=1$.

$\theta^{-1} \alpha \theta=\alpha \beta, \theta^{-1} \beta \theta=\beta ; \theta^{-1} \xi \theta=\xi \eta, \theta^{-1} \eta \theta=\eta ;[\theta, \zeta]=1 ; \theta^{2}=\beta$.

Write Out $(V)$ for Aut $(V) / \operatorname{Inn}(V)$. Then Out $(V)=\langle\bar{\eta}, \bar{\xi}, \bar{\theta}\rangle \times\langle\bar{\zeta}\rangle \cong D_{8} \times Z_{2}$ with $\langle\bar{\eta}\rangle$ as derived group.

The subgroup of Aut $(V)$ which sends each of $E$ and $H$ to themselves is $\langle\operatorname{Inn}(V), \xi, \eta, \zeta\rangle$. The subgroup of Aut $(V)$ which fixes $W$ elementwise is $\langle\operatorname{Inn}(V), \xi, \eta, \theta\rangle$. The subgroup of Aut $(V)$ which fixes elementwise the quotient $V \mid\langle w\rangle$ is $\langle\operatorname{Inn}(V), \zeta\rangle$.

Case I.1. $m=1$. I.e. $B=W$. $W$ is central in $C$, so $W \in \mathrm{SCN}(C) \Rightarrow C=W$. So $G / W \hookrightarrow$ Aut $(V)$; hence $|G| \leqq 4 \cdot 64=2^{8}$.

(ix) In Case I.1, suppose $\eta_{0}, \zeta_{0} \in G$ induce (by conjugation) the automorphisms $\eta$, $\zeta$ on $V$; suppose $x, y \in V$. Then $\left[x \eta_{0}, y \zeta_{0}\right] \equiv f \bmod W$.

Proof.

$$
\begin{aligned}
{\left[x \eta_{0}, y \zeta_{0}\right] } & =\left[x \eta_{0}, \zeta_{0}\right]\left[x \eta_{0}, y\right]^{\zeta} \\
& =\left[x, \zeta_{0}\right]^{\eta}\left[\eta_{0}, \zeta_{0}\right][x, y]^{\eta \zeta}\left[\eta_{0}, y\right]^{\zeta} \\
& =\left(x^{-1} x^{\zeta}\right)^{\eta}\left[\eta_{0}, \zeta_{0}\right][x, y]^{\eta \zeta}\left(y^{-\eta} y\right)^{\zeta}
\end{aligned}
$$


Since $[V, \zeta]=\langle w\rangle$ and $[V, \eta]=\left\langle w_{1}\right\rangle$, we have

$$
\left[x \eta_{0}, y \zeta_{0}\right] \equiv\left[\eta_{0}, \zeta_{0}\right] \bmod W .
$$

But in Aut $V,[\eta, \zeta]=\beta$; so in $G,\left[\eta_{0}, \zeta_{0}\right] \equiv f \bmod C_{G}(V)=W$.

Let $T$ be a five-generator subgroup of $G$, i.e., $[T: \Phi(T)]=2^{5}$. Then $2^{5} \leqq|T| \leqq 2^{8}$. $[T: T \cap V]=[T V: V] \leqq \mid$ Out $(V) \mid=16$.

Case I.1a. $T$ is elementary of rank 5. $[T: T \cap V] \leqq 16 \Rightarrow[T \cap V] \geqq 2 . T \cap V$ is elementary; the only elementary subgroups of $V$ are the subgroups of $E$ and $H$, so $T \cap V \subseteq E$ or $H$.

Suppose $|T \cap V|=8$. Then $T \cap V=E$ or $H$, and $T / T \cap V \cong E_{4}$. Now $T$ normalizes $T \cap V$, so $A_{T V}(V) \subseteq\langle\operatorname{Inn}(V), \xi, \eta, \zeta\rangle$; also $T$ centralizes $W$, so $\boldsymbol{A}_{T V}(V)$ $\subseteq\langle\operatorname{Inn}(V), \xi, \eta\rangle$. But

$$
E_{4} \cong T / T \cap V \cong T V / V \rightarrow\langle\xi, \bar{\eta}\rangle .
$$

Since $\langle\xi, \bar{\eta}\rangle$ is a four-group, we must have $A_{T V}(V)=\langle\operatorname{Inn}(V), \bar{\xi}, \bar{\eta}\rangle$. So $T$ contains an element $x \eta_{0}$ where $x \in V$ and $\eta_{0} \in G$ induces the automorphism $\eta$ on $V$.

$$
\begin{aligned}
& {\left[e, x \eta_{0}\right] \equiv\left[e, \eta_{0}\right] \bmod \langle w\rangle=e^{-1} e^{\eta}=w_{1} .} \\
& {\left[h, x \eta_{0}\right] \equiv\left[h, \eta_{0}\right] \bmod \langle w\rangle=h^{-1} h^{\eta}=w_{1} .}
\end{aligned}
$$

So whether $T \cap V$ is $E$ or $H, \Phi(T)$ contains an element congruent to $w_{1} \bmod \langle w\rangle$; but $T$ was elementary.

Suppose $|T \cap V|=4$. Then

$$
E_{8} \cong T / T \cap V \cong T V / V \hookrightarrow \text { Out }(V) \cong D_{8} \times Z_{2}
$$

Out $(V)$ has two $E_{8}$ 's, namely $\langle\bar{\xi}, \bar{\eta}, \bar{\zeta}\rangle$ and $\langle\bar{\theta}, \bar{\eta}, \bar{\zeta}\rangle$. So $T$ must contain elements $x \eta_{0}, y \zeta_{0}$ where $\eta_{0}, \zeta_{0} \in G$ induce $\eta, \zeta$ on $V$, and $x, y \in V$. But then $\Phi(T) \neq 1$ by (ix).

Suppose $|T \cap V|=2$. Then

$$
E_{16} \cong T / T \cap V \cong T V / V \rightarrow \text { Out }(V) \cong D_{8} \times Z_{2},
$$

which is impossible. Therefore Case I.1a is impossible.

Case I.1b. $|T|=2^{6}$ and $|\Phi(T)|=2$.

$[T: T \cap V] \leqq 16 \Rightarrow|T \cap V| \geqq 4$.

Suppose $T \supseteq V$. Then $\Phi(T)=\langle w\rangle$ and so $T / V \cong E_{4}$. Also $A_{T}(V)$ centralizes $V \mid\langle w\rangle$, so $A_{T}(V) \subseteq\langle\operatorname{Inn}(V), \zeta\rangle$. So

$$
E_{4} \cong T / V \hookrightarrow\left\langle\bar{\zeta} \cong Z_{2},\right.
$$

which is impossible.

Suppose $|T \cap V|=8$. Then $T \cap V$ is a maximal subgroup of $V$, so is $E, H, F=\langle f, W\rangle$, or one of four $D_{8}$ 's of $V$. If $T \cap V$ is not elementary, then $\Phi(T)=\langle w\rangle$ and so $T / T \cap V$ is elementary; so

$$
E_{8} \cong T / T \cap V \cong T V / V \hookrightarrow \text { Out }(V) \cong D_{8} \times Z_{2} .
$$

Out $(V)$ has two $E_{8}$ 's, both of which contain $\bar{\eta}$ and $\xi$. So $T$ contains elements 
$x \eta_{0}, y \zeta_{0}$ as in (ix). Therefore by (ix), $\Phi(T)$ contains an element of order 4 . But this contradicts $|\Phi(T)|=2$. If $T \cap V$ is elementary, then $T \cap V=E$ or $H$. $T$ normalizes $T \cap V$, so $A_{T V}(V) \subseteq\langle\operatorname{Inn}(V), \xi, \eta, \zeta\rangle$. Therefore

$$
T V \mid V \hookrightarrow\langle\bar{\xi}, \bar{\eta}, \bar{\zeta}\rangle \text {. }
$$

But $[T V: V]=[T: T \cap V]=8$. So $A_{T V}(V)=\langle\operatorname{Inn}(V), \xi, \eta, \zeta\rangle$ and $T$ contains elements $x \eta_{0}, y \zeta_{0}$ as in (ix); so by (ix), $\Phi(T)$ contains an element of order 4, contrary to $|\Phi(T)|=2$.

Suppose $|T \cap V|=4$. Then

$$
16=[T: T \cap V]=[T V: V]=\text { Out }(V) .
$$

So $\boldsymbol{A}_{T V}(V)=$ Aut $(V)$.

We show $T \cap V=W$. For $T$ normalizes $T \cap V$. If $T \cap V$ is cyclic then $T \cap V=\langle f\rangle$ or $\left\langle f w_{1}\right\rangle$. However, $T$ contains an element $x \xi_{0}$, where $x \in V$, and $\xi_{0} \in G$ induces the automorphism $\xi$ of $V$; and $x \xi_{0}$ exchanges the two subgroups $\langle f\rangle,\left\langle f w_{1}\right\rangle$ of $V$. So $T \cap V$ is elementary and so $T \cap V \subseteq E$ or $H$. $T$ contains an element $x \theta_{0}$, where $x \in V$, and $\theta_{0} \in G$ induces the automorphism $\theta$ of $V$; if $T \cap V \notin W$, then $x \theta_{0}$ would not normalize $T \cap V$ since $x \theta_{0}$ exchanges $E$ and $H$.

$T$ contains an element $x \zeta_{0}$, where $x \in V$, and $\zeta_{0} \in G$ induces the automorphism $\zeta$ of $V$. So $\Phi(T)$ contains $\left[w_{1}, x \zeta_{0}\right]=\left[w_{1}, \zeta_{0}\right]=w$. So $\Phi(T)=\langle w\rangle$, and so $T / T \cap V$ is elementary. But

$$
T / T \cap V \cong T V / V \cong \text { Out }(V) \cong D_{8} \times Z_{2} .
$$

Therefore Case I.1b is impossible.

Case I.1c. $|T|=2^{7}$ and $|\Phi(T)|=4$.

$[T: T \cap V] \leqq 16 \Rightarrow|T \cap V| \geqq 8$.

Suppose $T \supseteq V$. Then $[T: V]=8$ and so $A_{T}(V)$ is a maximal subgroup of Aut $(V)$; and $\boldsymbol{A}_{T}(V)$ contains Inn $(V)$. Every maximal subgroup of Out $(V)$ contains $\bar{\eta}$; so $T$ contains an element $\eta_{0}$ which induces $\eta$ on $V$. Then $\Phi(T)$ contains $\left[e, \eta_{0}\right]$ $=e^{-1} e^{\eta}=w_{1}$. Also $\Phi(T) \supseteq \Phi(V)=\langle w\rangle$. So $\Phi(T)=W$, and $T / V \cong E_{8}$. Out $(V)$ contains two $E_{8}$ 's, both of which contain $\langle\bar{\eta}, \zeta\rangle$. So $T$ contains elements $\eta_{0}$, $\zeta_{0}$ inducing $\eta, \zeta$ on $V$; by (ix), $\Phi(T) \supseteq\langle f, W\rangle$. This contradicts $|\Phi(T)|=4$.

Suppose $|T \cap V|=8$. Then

$$
16=[T: T \cap V]=[T V: V]
$$

and so $\boldsymbol{A}_{T V}(V)=$ Aut $(V)$. $T$ contains elements $x \eta_{0}, y \zeta_{0}$ as in (ix), so by (ix), $\Phi(T)$ contains an element of the coset $f W$. So $|\Phi(T) \cap V| \geqq 4$; hence $\Phi(T) \subseteq V$, and $T / T \cap V$ is elementary. But

$$
T / T \cap V \cong T V / V \cong \text { Out }(V) \cong D_{8} \times Z_{2} .
$$

Therefore Case I.1c is impossible.

Case I.1d. $|T|=2^{8}$ and $|\Phi(T)|=8$. Then $T=G$ and $G$ induces all of Aut $(V)$ on $V$. $\Phi(G) \geqq W$ by (v). Also $G$ contains $\eta_{0}, \zeta_{0}$ inducing $\eta$, $\zeta$ on $V$, so $\Phi(G) \supseteq\langle f, W\rangle$ by 
(ix). By hypothesis, $|\Phi(G)|=8$; so $\Phi(G)=\langle f, W\rangle$ and therefore $G / V$ is elementary. But

$$
G / V \cong \text { Out }(V) \cong D_{8} \times Z_{2}
$$

Therefore Case I.1d is impossible.

Case I.2. $m=2$. Then $\left\langle b^{2}\right\rangle=\mho^{1}(B) \triangleleft G$, so $b^{2}$ is central in $G$. So $W=\left\langle w, b^{2}\right\rangle$ is central in $G$. We may take $w_{1}=b^{2}$ in our computation of Aut $(V)$.

$C / B$ acts faithfully on $B$ and stabilizes the chain $B>W>1$, so is at most elementary of order 4.

(x) In Case I.2, $\Phi(C) \subseteq W$; and hence

$$
\begin{aligned}
\Phi(C V) & =\Phi(C) \Phi(V) \quad \text { since } C \text { centralizes } V \\
& =W .
\end{aligned}
$$

Proof. By Lemma FB, it suffices to show $x^{2} \in W$ for all $x \in C$.

$B^{2} \subseteq W$. Let $x \in C, x \notin B . x^{2} \in B$ since $C / B$ is elementary. Now $C_{B}(x)=W$ since $x$ induces a nontrivial automorphism $\in$ the stability group of $B>W>1$. $x$ centralizes $x^{2}$, so $x^{2} \in W$.

(xi) In Case I.2, $[C: B] \leqq 2$.

Proof. If $[C: B]=4$, then $[C V: \Phi(C V)]=2^{5}$; and $C V \triangleleft G$. But this contradicts (vi).

Case I.2.1. $C=B$. Then $|C V|=32$. $W$ is central in $G$, so $|G| \geqq 2^{8}$ by (vii), and also $A_{G}(V) \subseteq\langle\operatorname{Inn}(V), \xi, \eta, \theta\rangle . G / C V \hookrightarrow$ Out $(V)$. So we must have $|G|=2^{8}$ and $\boldsymbol{A}_{G}(V)=\langle\operatorname{Inn}(V), \xi, \eta, \theta\rangle$.

If $T$ is a subgroup of $G$ with $[T: \Phi(T)]=2^{5}$, then by (v) and (vi), $|T|=2^{5}$ or $2^{6}$ and $|\Phi(T)|=1$ or 2 . Since $\Phi(C V)=W, T \ddagger C V .[T: T \cap C V]=[T C V: C V] \leqq 2^{3}$.

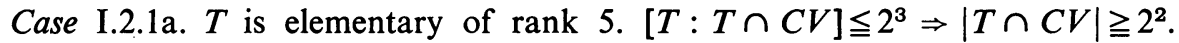
Since $C V-V$ contains no involutions, the only elementary subgroups of $C V$ are the subgroups of $E$ and $H$. So $T \cap C V \subseteq E$ or $H$.

Suppose $T \cap C V=E$ or $H$. Since $T$ normalizes $T \cap C V, A_{T C V}(V) \subseteq\langle\operatorname{Inn}(V), \xi, \eta\rangle$. But $4=[T: T \cap C V]=[T C V: C V]$ and so $A_{T C V}(V)=\langle\operatorname{Inn}(V), \xi, \eta\rangle$. So $T$ contains an element $x \eta_{0}$, where $x \in V$, and $\eta_{0} \in G$ induces the automorphism $\eta$ of $V$.

$$
\begin{aligned}
& {\left[e, x \eta_{0}\right] \equiv\left[e, \eta_{0}\right] \quad \bmod \langle w\rangle=e^{-1} e^{\eta}=w_{1} .} \\
& {\left[h, x \eta_{0}\right] \equiv\left[h, \eta_{0}\right] \quad \bmod \langle w\rangle=h^{-1} h^{\eta}=w_{1} .}
\end{aligned}
$$

So whether $T \cap C V$ is $E$ or $H, \Phi(T)$ contains an element congruent to $w_{1} \bmod \langle w\rangle$; but $T$ was elementary.

Suppose $|T \cap C V|=4$. Then

$$
E_{8} \cong T / T \cap C V \cong T C V / C V \hookrightarrow\langle\xi, \bar{\eta}, \bar{\theta}\rangle \cong D_{8},
$$

which is impossible. So Case I.2.1a is impossible.

Case I.2.1b. $|T|=2^{6}$ and $|\Phi(T)|=2$.

$$
[T: T \cap C V] \leqq 2^{3} \Rightarrow|T \cap C V| \geqq 8, \quad T \ddagger C V \Rightarrow|T \cap C V| \leqq 16 .
$$


Suppose $|T \cap C V|=16$. Then $T \cap C V$ is a maximal subgroup of $C V$, so is the inverse image in $C V$ of one of the seven hyperplanes of $C V / W$. If we write $\bar{C} \bar{V}$ for $C V / W$, then $\bar{C} \bar{V}=\langle\bar{b}, \bar{f}, \bar{e}\rangle$ and the seven hyperplanes are:
1. $\langle\bar{b}, \bar{f}\rangle$
2. $\langle\bar{b}, \bar{f} \bar{e}\rangle$
3. $\langle\bar{b}, \bar{e}\rangle$
4. $\langle\bar{b} \bar{f}, \bar{e}\rangle$
5. $\langle\bar{b} \bar{f}, \bar{e} \bar{f}\rangle$
6. $\langle\bar{b} \bar{e}, \bar{f}\rangle$
7. $\langle\bar{e}, \bar{f}\rangle=\bar{V}$.

The Frattini subgroups of their inverse images are:

1. $\left\langle b^{2}, f^{2}\right\rangle=W$

2. $\left\langle w_{1}\right\rangle$

3. $\left\langle w_{1}\right\rangle$

4. $\left\langle(b f)^{2},[b f, e]\right\rangle=\left\langle w_{1} w, w\right\rangle=W$

5. $W$

6. $\left\langle(b e)^{2},[b e, f]\right\rangle=\left\langle w_{1}, w\right\rangle=W$

7. $\langle w\rangle$.

Since $|\Phi(T)|=2$, we must have $T \cap C V=\langle b, e f, W\rangle$ or $\langle b, e, W\rangle$ or $V$.

If $T \cap C V=V$, then $\Phi(T)=\langle w\rangle$, and

$$
E_{4} \cong T / T \cap C V \cong T C V / C V \hookrightarrow\langle\xi, \bar{\eta}, \bar{\theta}\rangle \cong D_{8}
$$

Every four-group of $\langle\bar{\xi}, \bar{\eta}, \bar{\theta}\rangle$ contains $\bar{\eta}$; so $T$ contains an element $x \eta_{0}$, where $x \in C V$, and $\eta_{0} \in G$ induces the automorphism $\eta$ on $V$. Then $\Phi(T)$ contains $\left[e, x \eta_{0}\right] \equiv\left[e, \eta_{0}\right] \bmod \langle w\rangle \equiv w_{1} \bmod \langle w\rangle$. So $\Phi(T) \supseteq W$, contrary to $|\Phi(T)|=2$.

Therefore $T \cap C V$ is $\langle b, E\rangle$ or $\langle b, H\rangle ; \Phi(T)=\left\langle w_{1}\right\rangle$.

$T$ normalizes $T \cap V=E$ or $H$; so $A_{T C V}(V) \subseteq\langle\operatorname{Inn}(V), \xi, \eta\rangle$. Also

$$
4=[T: T \cap C V]=[T C V: C V] \hookrightarrow\langle\xi, \bar{\eta}\rangle ;
$$

so $\boldsymbol{A}_{T C V}(V)=\langle\operatorname{Inn}(V), \xi, \eta\rangle$ and $T$ contains elements $x \xi_{0}, y \eta_{0}$, where $x, y \in C V$, and $\xi_{0}, \eta_{0} \in G$ induce $\xi, \eta$ on $V$.

If $T \cap V=E$, then $\Phi(T)$ contains $\left[e, x \xi_{0}\right]=\left[e, \xi_{0}\right][e, x]^{\xi}=[e, x]^{\xi}=w$ or 1 , according as $x$ does not or does centralize $e$. Also $\Phi(T)$ contains $\left[e, y \eta_{0}\right]=\left[e, \eta_{0}\right][e, y]^{n}$ $=w_{1}[e, y]^{\eta}=w_{1} w$ or $w_{1}$, according as $y$ does not or does centralize $e$. But $\Phi(T)=\left\langle w_{1}\right\rangle$, so $x$ and $y$ must centralize $e . C_{C V}(e)=\langle B, E\rangle=T \cap C V$; so we may take $x=y=1$.

If $T \cap V=H$, then $\Phi(T)$ contains the elements

$$
\begin{aligned}
& {\left[h, x \xi_{0}\right]=\left[h, \xi_{0}\right][h, x]^{\xi}=w_{1} w \text { or } w_{1}} \\
& {\left[h, y \eta_{0}\right]=\left[h, \eta_{0}\right][h, y]^{\eta}=w_{1} w \text { or } w_{1}}
\end{aligned}
$$

according as $x, y$ do not or do centralize $h$. Since $\Phi(T)=\left\langle w_{1}\right\rangle, x, y \in C_{C V}(h)$ $=\langle B, H\rangle=T \cap C V$; so again we may take $x=y=1$.

So $T=\left\langle b, w, e\right.$ or $\left.h, \xi_{0}, \eta_{0}\right\rangle ; \Phi(T)=\left\langle w_{1}\right\rangle$. 
Consider $R=\left\langle B, \xi_{0}, \eta_{0}\right\rangle . G=\left\langle R, V, \theta_{0}\right\rangle$ where $\theta_{0} \in G$ induces $\theta$ on $V$. Now $\langle\operatorname{Inn}(V), \xi, \eta\rangle$ is an Abelian subgroup of Aut $(V)$; therefore in $G$,

$$
\left[V, \xi_{0}\right] \equiv\left[V, \eta_{0}\right]=\left[V, \xi_{0} \eta_{0}\right] \equiv 1 \bmod B=C_{G}(V) .
$$

Also $\left[\xi_{0}, \eta_{0}\right] \equiv 1 \bmod B$. So the three subgroups $\left\langle B, \xi_{0}\right\rangle,\left\langle B, \eta_{0}\right\rangle,\left\langle B, \xi_{0} \eta_{0}\right\rangle$ of $R$ all normal in $R V$.

We consider the action of $G$ on these three subgroups. $B \triangleleft G$. In Aut $(V)$, $[\eta, \theta]=1$ and $[\xi, \theta]=\eta$; so in $G$,

$$
\left[\eta_{0}, \theta_{0}\right] \equiv 1 \quad \text { and } \quad\left[\xi_{0}, \theta_{0}\right] \equiv \eta_{0} \bmod B .
$$

Therefore $\theta_{0}$ normalizes $R$ and $\left\langle B, \eta_{0}\right\rangle$, and exchanges $\left\langle B, \xi_{0}\right\rangle$ and $\left\langle B, \xi_{0} \eta_{0}\right\rangle$.

Since $\Phi(T)=\left\langle w_{1}\right\rangle=\left\langle b^{2}\right\rangle, \eta_{0}$ either centralizes or inverts $b$. If $\eta_{0}$ centralizes $b$, then $\left\langle B, \eta_{0}\right\rangle$ is Abelian of order 16 and exponent 4 and has Frattini subgroup of order 2; hence $\Omega_{1}\left(\left\langle B, \eta_{0}\right\rangle\right) \cong E_{8}$ and is normal in $G$. Therefore $\eta_{0}$ inverts $b$.

Also $\xi_{0}$ and $\xi_{0} \eta_{0}$ either centralize or invert $b$. So of the three elements $\xi_{0}, \eta_{0}$, and $\xi_{0} \eta_{0}$, two must invert $b$ and one must centralize $b$. The one centralizing $b$ will (with $B$ ) generate an Abelian group, the others will generate non-Abelian groups. Two of these groups are $G$-conjugate and the third normal in $G$; so the Abelian one must be $\left\langle B, \eta_{0}\right\rangle$, contrary to the above. Therefore $|T \cap C V| \neq 16$.

Suppose $|T \cap C V|=8$. Then

$$
8=[T: T \cap C V]=[T C V: C V]=[G: C V],
$$

so $T C V=G$ and $T / T \cap C V \cong T C V / C V \cong D_{8}$. Therefore $\Phi(T) \nsubseteq T \cap C V$. Since $|\Phi(T)|=2, \Phi(T) \cap(T \cap C V)=1$ and so $T \cap C V$ is elementary, so $\subseteq E$ or $H$. $|T \cap C V|=8$, so $T \cap C V=E$ or $H$. But $T$ contains an element $x \theta_{0}$, where $x \in C V$, and $\theta_{0} \in G$ induces $\theta$ on $V ; x \theta_{0}$ exchanges $E$ and $H$. This is impossible since $T \cap C V \triangleleft T$.

Case I.2.2. $[C: B]=2$. Then $Z(C)=W ; \Phi(C V)=\Phi(C) \Phi(V)$ (since $C$ centralizes $V)=W ; Z(C V)=W \cdot|C V|=64$. By (vii), $|G| \geqq 2^{8}$; hence $[G: C V] \geqq 4$.

$G$ normalizes $C V$ and $W$, so acts on $C V / W$.

(xi) In Case I.2.2, $C_{G}(C V / W)=C V$.

Proof. If not, let $R$ be a subgroup of $G$ with $C_{G}(C V / W) \supseteq R>C V,[R: C V]=2$, and $R \triangleleft G . R$ stabilizes the chain $C V>W>1$; the stability group of this chain is elementary, so $\Phi(R)$ centralizes $C V$. But also $\Phi(R) \subseteq C V$; so $\Phi(R) \subseteq Z(C V)=W$. So $\Phi(R)=W$, and $[R: \Phi(R)]=2^{5}$; this contradicts (vi).

So $G / C V$ acts faithfully on $C V / W$. But $C \triangleleft G, V \triangleleft G$; so as $G$-module, $C V / W=(C / W) \times(V / W)$ is the direct product of two four-groups; so $[G: C V] \leqq 4$. Hence $[G: C V]=4$ and $G$ contains elements $\lambda, \mu$ inducing the following automorphisms of $C V / W: \lambda$ centralizes $V / W$ and $b W$, and sends $c W$ to $c b W$ for $c \in C-B$; $\mu$ centralizes $C / W$ and $f W$, and sends $e W$ to ef $W=h W$.

Since $[G: C V]=4,|G|=2^{8}$. By (vi), $G$ and all its maximal subgroups are fourgenerator groups. So if $G$ has a subgroup $T$ with $[T: \Phi(T)]=2^{5}$, then either $T$ is 
elementary of order $2^{5}$, or $|T|=2^{6}$ and $|\Phi(T)|=2$. Since $\Phi(C V)=W, T$ $\mid C V$. $[T: T \cap C V]=[T C V: C V] \leqq 4$.

(xii) In Case I.2.2, if $G$ has a subgroup $T$ with $[T: \Phi(T)]=2^{5}$, then $G$ has a subgroup $T$ with $[T: \Phi(T)]=2^{5}$ and $T \supseteq W$.

Proof. Let $T$ be a subgroup of $G$ with $[T: \Phi(T)]=2^{5}$ and $|T|$ minimal. $W$ is elementary and central in $G$, so $\Phi(T W)=\Phi(T)$. If $W \nsubseteq T$, consider $T W$; there is a subgroup $L$ of $T W$ with $\langle\Phi(T), W\rangle \leqq L$ and $[L: \Phi(T)]=2^{5}$. Then $|L|=|T|$; so by minimality of $|T|,[L: \Phi(L)]=2^{5}$.

Case I.2.2a. $T$ is elementary of rank $5, T \supseteq W$.

$[T: T \cap C V] \leqq 4 \Rightarrow|T \cap C V| \geqq 8$.

Suppose $|T \cap C V|=32$; then $T \subseteq C V$ is the inverse image in $C V$ of a hyperplane of $C V / W=\langle\bar{c}, \bar{b}, \bar{f}, \bar{e}\rangle$. Any hyperplane intersects the two-dimensional subspace $\langle\bar{b}, \bar{f}\rangle$ of $C V / W$; so $|T \cap\langle b, f, W\rangle| \geqq 8$. But $\langle b, f, W\rangle=\langle b, f\rangle \cong Z_{4} \times Z_{4}$; so $T$ is not elementary.

Suppose $|T \cap C V|=16$. Then $[T: T \cap C V]=2$, so $T$ contains an element $x \lambda$, $x \mu$, or $x \lambda \mu$ for $x \in C V$. If $T$ contains $x \lambda$, then $T / W$ elementary $\Rightarrow(T \cap C V) / W$ $\subseteq C_{C V / W}(\lambda)=\langle\bar{b}, \bar{V}\rangle$. If $T$ contains $x \mu$, then $T / W$ elementary $\Rightarrow(T \cap C V) / W \subseteq$ $C_{C V / W}(\mu)=\langle\bar{f}, \bar{C}\rangle$. If $T$ contains $x \lambda \mu$, then $T / W$ elementary $\Rightarrow(T \cap C V) / W$ $\subseteq \boldsymbol{C}_{C V / W}(\lambda \mu)=\langle\bar{b}, \bar{f}\rangle$. Now $\langle b, V\rangle$ and $\langle f, C\rangle$ are of order 32 and $\langle b, f\rangle$ is of order 16; so in any of these three cases, $T \cap C V$ contains a maximal subgroup of $\langle b, f\rangle \cong Z_{4} \times Z_{4}$, and so $T$ is not elementary.

Suppose $|T \cap C V|=8$. Then $[T: T \cap C V]=4$, so $T$ contains elements $x \lambda, y \mu$ for some $x, y \in C V . \quad T / W$ elementary $\Rightarrow(T \cap C V) / W \subseteq C_{C V / W}(\langle\lambda, \mu\rangle)=\langle\bar{b}, \bar{f}\rangle$. So $T \cap C V$ is a maximal subgroup of $\langle b, f\rangle \cong Z_{4} \times Z_{4}$; so $T$ is not elementary. So Case I.2.2a is impossible.

Case I.2.2b. $|T|=2^{6}$ and $|\Phi(T)|=2$.

$[T: T \cap C V] \leqq 4 \Rightarrow|T \cap C V| \geqq 16$.

Suppose $|T \cap C V|=32$. Then $[T: T \cap C V]=2$ and $T$ contains an element $x \lambda$, $x \mu$, or $x \lambda \mu$ for some $x \in C V$.

Suppose $x \lambda \in T$. Then $(T \cap C V) / W \subseteq C_{C V / W}(\lambda)=\langle\bar{b}, \bar{V}\rangle$. For if not, $(T \cap C V) / W$ contains $\bar{c} \bar{a}$ for some $\bar{a} \in\langle\bar{b}, \bar{V}\rangle$, and $[\bar{c} \bar{a}, \bar{x} \lambda]=\bar{b}$; so $\Phi(T)$ contains an element of the coset $b W$; but this contradicts $|\Phi(T)|=2$. So $T \cap C V \subseteq\langle b, V, W\rangle$; since $|T \cap C V|=32, T \cap C V=\langle b, V, W\rangle=\langle b, V\rangle$. But $\Phi(\langle b, V\rangle)=W$, contradicting $|\Phi(T)|=2$.

Suppose $x \mu \in T$. Then $(T \cap C V) / W \subseteq C_{C V / w}(\mu)=\langle\bar{c}, \bar{f}\rangle$. For if not, $(T \cap C V) / W$ contains $\bar{e} \bar{a}$ for some $\bar{a} \in\langle\bar{c}, \bar{f}\rangle$, and $[\bar{e} \bar{a}, \bar{x} \bar{\mu}]=f$; so $\Phi(T)$ contains an element of $f W$, contrary to $|\Phi(T)|=2$. So $T \cap C V=\langle C, f\rangle$; but $\Phi(\langle c, f\rangle)=W$, contradicting $|\Phi(T)|=2$.

Suppose $x \lambda \mu \in T$. Then $(T \cap C V) / W \subseteq C_{C V / w}(\lambda \mu)=\langle\bar{b}, \bar{f}\rangle$. For if not, then $(T \cap C V) / W$ contains one of $\bar{c} \bar{a}, \bar{e} \bar{a}, \bar{c} \bar{e} \bar{a}$ for $\bar{a} \in\langle\bar{b}, \bar{f}\rangle$; correspondingly, $[\bar{c} \bar{a}, \bar{x} \lambda \bar{\mu}]=\bar{b},[\bar{e} \bar{a}, \bar{x} \lambda \bar{\mu}]=\bar{f},[\bar{c} \bar{e} \bar{a}, \bar{x} \lambda \bar{\mu}]=\bar{b} \bar{f}$; and $\Phi(T)$ contains an element of $b W$, $f W$, or $b f W$, contrary to $|\Phi(T)|=2$. So $T \cap C V \subseteq\langle b, f\rangle$, contrary to $|T \cap C V|=32$. 
Suppose $|T \cap C V|=16$. Then $[T C V: C V]=4$, so $T$ contains elements $x \lambda$ and $y \mu$ for $x, y \in C V$. As above, $(T \cap C V) / W \subseteq\langle\bar{b}, \bar{f}\rangle$, so $T \cap C V=\langle b, f\rangle$; but $\Phi(\langle b, f\rangle)=W$, contrary to $|\Phi(T)|=2$. So Case I.2.2b is impossible.

Case I.3. $m \geqq 3$. Let $d=b^{2^{m-2}} ;\langle d\rangle=\mho^{m-2}(B)$, so $\langle d\rangle \triangleleft G . d^{2}=w_{1}$ is central in $G$, so $W$ is central in $G$.

(xiii) In Case I.3, if $G$ has a subgroup $T$ with Frattini quotient of rank 5 , then so does $G /\left\langle w_{1}\right\rangle$.

Proof. If $w_{1} \notin T$, then $G /\left\langle w_{1}\right\rangle$ contains an isomorphic copy of $T$. If $w_{1} \in \Phi(T)$, then $T /\left\langle w_{1}\right\rangle$ has Frattini quotient isomorphic to that of $T$. If $w_{1} \in T$ but $w_{1} \notin \Phi(T)$, then $d \notin T$; let $D=\langle d\rangle . \Phi(T D)=\langle\Phi(T), \Phi(D),[T, D]\rangle$ (by Lemma FC) = $\left\langle\Phi(T), w_{1}\right\rangle$. So $[T D: \Phi(T D)]=[T: \Phi(T)]$, and $T D /\left\langle w_{1}\right\rangle$ has Frattini quotient of rank 5 .

So by induction on $|G|, G /\left\langle w_{1}\right\rangle$ has a normal $E_{8}$, say $K /\left\langle w_{1}\right\rangle$.w $w\left\langle w_{1}\right\rangle$ and $d\left\langle w_{1}\right\rangle$ are central involutions of $G /\left\langle w_{1}\right\rangle$, so we may take $K \supseteq\langle d, W\rangle . \Phi(K)=\left\langle w_{1}\right\rangle$ and $K$ is of order 16 and exponent 4 ; so $K$ is non-Abelian because otherwise $\Omega_{1}(K)$ would be a normal $E_{8}$ of $G$. Therefore $Z(K)=W . K \cap V \triangleleft G$ and $\Phi(K \cap V) \subseteq \Phi(K)$ $\cap \Phi(V)=1$; so $K \cap V=W$ and $|K V|=64$.

(xiv) In Case I.3, $Z(K V)=W$.

Proof. Let $a \in K, v \in W$ with $a v \in Z(K V)$. Then $a v=(a v)^{d}=a^{d} v$ since $d$ centralizes $V$. So $a \in C_{K}(D)=\langle d, W\rangle$. Therefore for $x \in V$, $a v=(a v)^{x}=a^{x} v^{x}=a v^{x}$ since $\langle d, W\rangle$ centralizes $V$. So $v \in Z(V)=W$; so $a v \in Z(K)=W$.

$G$ acts on $K V / W$, since $K V$ and $W$ are normal in $G$.

(xv) In Case I.3, $C_{G}(K V / W)=K V$.

Proof. If not, take a subgroup $R$ of $G$ such that $C_{G}(K V / W) \supseteq R>K V,[R: K V]=2$, and $R \triangleleft G$. $R$ stabilizes the chain $K V>W>1$, and the stability group of this chain is elementary; so $\Phi(R)$ centralizes $K V$. But also $\Phi(R) \subseteq K V$, so $\Phi(R) \subseteq Z(K V)=W$. So $\Phi(R)=W$ and $[R: \Phi(R)]=2^{5}$; this contradicts (vi).

So $G / K V$ acts faithfully on $K V / W . K \triangleleft G$ and $V \triangleleft G$; so as $G$-module, $K V / W=(K / W) \times(V / W)$ is a direct product of four-groups each invariant under $G$. Therefore $[G: K V] \leqq 4$. By (vii), $|G| \geqq 2^{8}$; so we have $|K V|=2^{6},|G|=2^{8}$, and $[G: K V]=4$.

$G$ has no subgroups requiring 5 or more generators which contain $K V$. For by (vi), $G$ and all its maximal subgroups are four-generator groups; and $\Phi(K V)=W$ so $K V$ is a four-generator group.

Let $T$ be any subgroup of $G$. We will show $T K V$ requires at least as many generators as $T$ (i.e., $[T K V: \Phi(T K V)] \geqq[T: \Phi(T)])$. This will prove that every subgroup of $G$ is a four-generator group, and so finish Case I.

$W$ is elementary and central in $G$, so $\Phi(T W)=\Phi(T)$ and hence $T W$ requires at least as many generators as $T$. So we will be done if we can show that $(T W) K V$ requires at least as many generators as $T W$; i.e., we may assume $T \supseteq W$.

We will now show $[T D: \Phi(T D)] \geqq[T: \Phi(T)]$. For $\Phi(T D)=\langle\Phi(T), " \Phi(D),[T, D]\rangle$ (by Lemma FC) $=\left\langle\Phi(T), w_{1}\right\rangle$. If $T D \neq T$ then $[\Phi(T D): \Phi(T)] \leqq 2$ and $[T D: T]=2$, 
so $[T D: \Phi(T D)] \geqq[T: \Phi(T)]$. So we may assume that $T \supseteq D$, and therefore that $w_{1} \in \Phi(T)$.

We will now show $[T F: \Phi(T F)] \geqq[T: \Phi(T)]$. For $\Phi(T F)=\langle\Phi(T), \Phi(F),[T, F]\rangle$ $\subseteq\langle\Phi(T), w, W\rangle$ since $F, W \triangleleft G$ and $[F: W]=2$. Since $w_{1} \in \Phi(T),[\Phi(T F): \Phi(T)]$ $\leqq 2$, and the conclusion follows. So we may assume that $T \supseteq F$, and therefore that $W \subseteq \Phi(T)$.

We will now show $[T V: \Phi(T V)] \geqq[T: \Phi(T)] . \quad \Phi(T V)=\langle\Phi(T), \Phi(V),[T, V]\rangle$ $\subseteq\langle\Phi(T), F\rangle$ since $V, F \triangleleft G$ and $[V: F]=2$. Since $W \subseteq \Phi(T)$, $[\Phi(T V): \Phi(T)] \leqq 2$, and the conclusion follows. So we may assume $T \supseteq V$.

We now show $[T K: \Phi(T K)] \geqq[T: \Phi(T)]$, which will complete the argument. For $\Phi(T K)=\langle\Phi(T), \Phi(K),[T, K]\rangle \subseteq\langle\Phi(T), d, W\rangle$ since $K$ and $\langle d, W\rangle \triangleleft G$ and $[K:\langle d, W\rangle]=2$. Since $W \subseteq \Phi(T),[\Phi(T K): \Phi(T)] \leqq 2$, and the conclusion follows.

Case II. Every maximal subgroup $M$ of $G$ has $\mathrm{SCN}_{3}(M)$ empty. Every proper subgroup of $G$ is contained in some maximal subgroup, so is a four-generator group by induction on $|G|$. So we need only show $G$ is a four-generator group.

If $W$ is central in $G$, then $G$ is a four-generator group by (vi). Also $W \subseteq \Phi(G)$ by (v), so if $G$ is a counterexample to the theorem, then $[G: W] \geqq 2^{5}$, so $|G| \geqq 2^{7}$.

(xvi) If $|G|=2^{7}$ and $W$ is noncentral in $G$, then $G$ is a four-generator group.

Proof. Let $K=C_{G}(W) ;|K|=2^{6}$. By (vi) (applied to $K$ instead of $G$ ), $K$ is a fourgenerator group, so $|\Phi(K)| \geqq 4$. $W \subseteq \Phi(G)$, so if $\Phi(K) \neq W$ then $\langle\Phi(K), W\rangle \subseteq \Phi(G)$ $\Rightarrow|\Phi(G)| \geqq 8 \Rightarrow[G: \Phi(G)] \leqq 16$. So if $G$ is not a four-generator group, we must have $\Phi(K)=W$.

$\Phi(K)$ is generated by the squares of the elements of $K$ (by Lemma FB), so there is $x \in K, x \notin W$, with $x^{2}$ a noncentral involution of $G\left(x^{2} \in W\right)$. For this $x$, if $g \in G-K$ then $\left(x^{g}\right)^{2}=\left(x^{2}\right)^{g} \neq x^{2}$, so $x^{g} \neq x$ mod $W$. So there are elements $a, b \in K$ with $K=\left\langle a, b, x, x^{g}\right\rangle$; then $G=\langle a, b, x, g\rangle$.

Let $\Omega_{1}(Z(G))=\langle w\rangle ; w \in \Phi(G)$ since $W \subseteq \Phi(G)$. Therefore $G /\langle w\rangle$ requires as many generators as $G$; so by induction on $|G|, G /\langle w\rangle$ has a normal $E_{8}, V /\langle w\rangle$ say. $V \triangleleft G, \Phi(V)=\langle w\rangle$, and $V$ is of exponent 4 and order 16. $V$ is non-Abelian since otherwise $E_{8} \cong \Omega_{1}(V) \triangleleft G$.

$V \cong Q_{8} \times Z_{2}, D_{8} \times Z_{2}$, or $Q_{8} \circ Z_{4}$. For if $x, y \in V$ have $[x, y] \neq 1$, then $[x, y]=w$ and $R=\langle x, y\rangle \cong Q_{8}$ or $D_{8} . \quad V=R C_{V}(R)$. $\left|C_{V}(R) \cap R\right|=2$, so $\left|C_{V}(R)\right|=4$ and $C_{V}(R) \cong E_{4}$ or $Z_{4}$. If $E_{4}$, then $V \cong Q_{8} \times Z_{2}$ or $D_{8} \times Z_{2}$; if $Z_{4}$, then $V \cong Q_{8} \circ Z_{4} \cong D_{8}$ 。 $\boldsymbol{Z}_{4}$.

If $V \cong D_{8} \times Z_{2}$, then $G$ must permute the two $E_{8}$ 's $E$ and $H$ of $V$; then $N_{G}(E)=N_{G}(H)$ is a maximal subgroup of $G$ with $\mathrm{SCN}_{3}$ nonempty, contrary to assumption. So $V \nsubseteq D_{8} \times Z_{2}$.

(xvii) In Case II, if $V \cong Q_{8} \circ Z_{4}$ then $G$ is a four-generator group.

Proof. Write $V=Q \circ\langle z\rangle$ where $z$ is central of order $4, z^{2}=w$, and $i, j, k \in Q$ represent the nonidentity cosets of $\langle w\rangle$ in $Q \cong Q_{8}$. Then the cosets of $\langle w\rangle$ in $V$ are represented by $i, j, k ; z ; z i, z j, z k$. The cosets represented by $i, j, k$ are the only 
cosets consisting of noncentral elements of order 4 in $V$, so these cosets are permuted by $G$; hence $Q \triangleleft G . Q$ contains three $Z_{4}$ 's- $\langle i\rangle,\langle j\rangle$, and $\langle k\rangle$-which are permuted by $G$, so one-say $\langle i\rangle$-is normal in $G$. Also $\langle z\rangle=Z(V) \triangleleft G$.

Write $C=C_{G}(Q) . C \triangleleft G$ and $[G: C Q] \leqq 2$. If $\left.G\right\rangle C Q$ then $G=\langle C Q, g\rangle$ where $j^{g} \equiv k \bmod \langle w\rangle$.

By Lemma FA, there is $B \in \operatorname{SCN}(C)$ with $B \supseteq\langle z\rangle$ and $B \triangleleft G$. Every such $B$ is cyclic; for if not, consider $K=\left\langle\Omega_{2}(B), i\right\rangle . K$ is a normal Abelian subgroup of $G$ and contains the three involutions of $\Omega_{1}(B)$ and also the involution $z i$; so $\Omega_{1}(K) \triangleleft G$ and is of order $\geqq 8$.

Write $B=\langle b\rangle$ of order $2^{\xi} ; \xi \geqq 2$ since $z \in B$. If $\xi=2$ then $B \in \operatorname{SCN}(C) \Rightarrow|C| \leqq 8$ and $|G| \leqq 64$; then $G$ is a four-generator group since $W \subseteq \Phi(G)$. So $\xi \geqq 3$.

If $C=B$ then $C Q=\langle b, Q\rangle=\langle b, j, k\rangle$, and if $G\rangle C Q$ then $G=\langle b, j, g\rangle$. So we may assume $C>B$. Let $C \geqq R>B$ with $[R: B]=2$. $R$ is non-Abelian since $B \in \mathrm{SCN}(C)$. The only non-Abelian 2-groups with a cyclic subgroup of index 2 and order $2^{\xi}$ are dihedral, semidihedral, generalized quaternion, and $P(\xi)=$ $\left\langle c, b: b\right.$ has order $\left.2^{\xi}, c^{2}=1, c b c=b^{1+2^{\xi-1}}\right\rangle . P(\xi)$ has exactly three involutions, which (together with 1) constitute a characteristic four-group.

Let $C \geqq R>B$ with $[R: B]=2$ and $R \triangleleft G$. $R$ either centralizes or inverts $\mho^{1}(B)$ (whose order is $\geqq 4$ ). If $R$ centralizes $\mho^{1}(B)$, then $R \cong P(\xi)$ and its characteristic four-group $X$ is normal in $G$. $W \subseteq V, W \nsubseteq Z(V)$, and $X \leqq C$, so $X$ centralizes $W$ and $X \neq W$; so $X W$ is a normal $E_{8}$ of $G$. Therefore $R$ inverts $\mho^{1}(B)$.

We now show $[C: B] \leqq 2$. For if not, let $R, S$ be subgroups of $G$ with $C \geqq S>R>B, \quad[S: R]=2, \quad[R: B]=2, \quad$ and $R, S \triangleleft G$. If $S / B$ is cyclic, then $R / B=(S / B)^{2}$ so $R$ induces on $\mho^{1}(B)$ the square of the automorphism which $S$ induces on $\mho^{1}(B)$; but $R$ inverts $\mho^{1}(B)$ and inversion is not a square in Aut $\left(\mho^{1}(B)\right)$. So $S / B$ is a four-group. Two of the three subgroups properly between $S$ and $B$, invert $\mho^{1}(B)$, and the third centralizes $\mho^{1}(B)$. $G$ permutes these three subgroups so must normalize the one which centralizes $\mho^{1}(B)$, but this is impossible.

Therefore $C=\langle c, b\rangle$ for $c \in C-B$. $C Q=\langle c, b, j, k\rangle$; if $G>C Q$ then $G=$ $\left\langle c, b, j, j^{g}, g\right\rangle=\langle c, b, j, g\rangle$ for $g \in G-C Q$.

So if $G$ is a counterexample to the theorem, $V \cong Q_{8} \times Z_{2}$. Write $V=Q \times\left\langle w_{1}\right\rangle$, where $Q \cong Q_{8}$ has derived group $\langle w\rangle$, and $w_{1} \in W-\langle w\rangle$. Let $i, j, k$ represent the three nonidentity cosets of $\langle w\rangle$ in $Q . W=Z(V)$ and $V$ has three maximal subgroups containing $W$, namely $\langle W, i\rangle,\langle W, j\rangle$, and $\langle W, k\rangle$. These are permuted by $G$, so one-say $\langle W, i\rangle$-is normal in $G$.

Write $C=C_{G}(V) . C \triangleleft G$, and by Lemma FA, there is $B \in \operatorname{SCN}(C)$ with $B \geqq W$ and $B \triangleleft G$. Now $w$ is not a square in $B$; for if $x \in B$ has $x^{2}=w$, then $x \in \Omega_{2}(B)$, and $K=\left\langle\Omega_{2}(B), i, W\right\rangle$ is a normal Abelian subgroup of $G ; K$ contains the three involutions of $W$ and also the involution $x i$; so $\Omega_{1}(K) \triangleleft G$ and is of rank at least 3, so contains a normal $E_{8}$ of $G$.

So $B=\langle b\rangle \times\langle w\rangle$ where $b$ is of order $2^{m}, m \geqq 1$. If $m>1$ then $\left\langle b^{2^{m-1}}\right\rangle=\mho^{m-1}(B)$ $\triangleleft G$ and so $W=\left\langle b^{2^{m-1}}, w\right\rangle$ is central in $G$, so that $G$ is a four-generator group by 
(vi). So $m=1$ and $B=W . W \in \operatorname{SCN}(C)$ and $W$ central in $C \Rightarrow C=W$, so $G / W \hookrightarrow \operatorname{Aut}(V)$.

Aut $(V)$. An abstract $Q_{8} \times Z_{2}$, with $\langle w\rangle$ as Frattini subgroup, can be presented as $\left\langle x, y, z: x^{2}=w, y^{2}=w, y^{-1} x y=x w=x^{-1} ; z^{2}=1, z\right.$ central $\rangle$.

Now any $a, b, c$ such that:

(1) $a, b$ are of order 4 and are independent mod the center (there are 12.8 such ordered pairs $(a, b))$,

(2) $c$ is a central involution $\neq w$ (there are two such $c$ )

will generate $Q_{8} \times Z_{2}$ and satisfy the same relations as those given above for $x, y, z$. So the automorphisms of $Q_{8} \times Z_{2}$ are in one-one correspondence with the choices for $a, b, c$. So $\mid$ Aut $(V) \mid=12 \cdot 8 \cdot 2=3 \cdot 64$.

We exhibit the following automorphisms of $V=\left\langle i, j, k, w_{1}\right\rangle$ :

$I_{i}, I_{j}, I_{k}=$ inner automorphisms induced by $i, j, k$. (Then $I_{i} I_{j}=I_{k}$.)

$\alpha: i, w_{1} \rightarrow$ selves, $j \rightarrow j w_{1}, k \rightarrow k w_{1}$.

$\beta: j, w_{1} \rightarrow$ selves, $i \rightarrow i w_{1}, k \rightarrow k w_{1}$.

$\gamma: k, w_{1} \rightarrow$ selves, $i \rightarrow i w_{1}, j \rightarrow j w_{1}$. (Then $\alpha \beta=\gamma$.)

$\zeta: i, j, k \rightarrow$ selves, $w_{1} \rightarrow w w_{1}$.

$\theta: i, w_{1} \rightarrow$ selves, $j \rightarrow k, k \rightarrow j$.

Then $\langle\operatorname{Inn}(V), \alpha, \beta\rangle \cong E_{16}$.

$\zeta$ centralizes Inn $(V)$ and $\zeta^{2}=1 ; \zeta \alpha \zeta=I_{i} \alpha, \zeta \beta \zeta=I_{j} \beta, \zeta \gamma \zeta=I_{k} \gamma$.

$\theta^{2}=1, \theta$ centralizes $\zeta ; \theta I_{i} \theta=I_{i}, \theta I_{j} \theta=I_{k}=I_{i} I_{j}, \theta \alpha \theta=\alpha, \theta \beta \theta=\gamma=\alpha \beta$.

These automorphisms generate a full Sylow 2-subgroup of Aut $(V)$, namely the Sylow 2-subgroup stabilizing $\langle W, i\rangle$ and exchanging $\langle W, j\rangle$ and $\langle W, k\rangle$. We have chosen notation in $G$ so that $\langle W, i\rangle \triangleleft G$, which amounts to saying that $A_{G}(V)$ lies in this particular Sylow 2-subgroup of Aut $(V)$.

$G / W \rightarrow$ Aut $(V) \Rightarrow[G: W] \leqq 64,|G| \leqq 2^{8}$. So by (xvi), $|G|=2^{8}$ and $A_{G}(V)$ is the full Sylow 2-subgroup of Aut $(V)$. So $G$ contains elements $\alpha_{0}, \beta_{0}, \gamma_{0}, \zeta_{0}$ inducing the automorphisms $\alpha, \beta, \gamma, \zeta$ on $V$. Now in Aut $(V)$,

hence in $G$,

$$
[\alpha, \zeta]=I_{i}, \quad[\beta, \zeta]=I_{j}, \quad[\gamma, \zeta]=I_{k} ;
$$

$$
\left[\alpha_{0}, \zeta_{0}\right] \equiv i, \quad\left[\beta_{0}, \zeta_{0}\right] \equiv j, \quad\left[\gamma_{0}, \zeta_{0}\right] \equiv k \quad \bmod C_{G}(V)=W .
$$

$\Phi(G) \geqq W$ by (v), and so $\Phi(G) \supseteq\langle W, i, j, k\rangle=V$. So $|\Phi(G)| \geqq 16$ and $[G: \Phi(G)] \leqq 2^{4}$.

This finishes Case II, and hence finishes the proof of the theorem.

2. Some useful results for dealing with simple groups. In this section we present two useful lemmas, and also establish some notation and properties for Aut $\left(\boldsymbol{Z}_{4} \times \boldsymbol{Z}_{4}\right)$.

LEMMA A (THOMPSON'S TRANSFER THEOREM). Let $G$ be a finite group of even order with no subgroup of index 2; let $T$ be a Sylow 2-subgroup of $G$, and $M$ a maximal subgroup of $T$. Let $x \in G$ be an involution outside $M$. Then some $G$-conjugate of $x$ lies in $M$. 
Proof. Consider the representation $G^{*}$ of $G$ as a permutation-group on the set $\{M y: y \in G\}$ of right cosets of $M$ in $G . x^{*}$ is represented by a product of transpositions; the fixed points of $x^{*}$ are the cosets $M y$ with $M y x=M y$, i.e., $y x y^{-1} \in M$. If no $y x y^{-1}$ lies in $M$, then $x^{*}$ is a product of an odd number of transpositions. Therefore the intersection of $G^{*}$ with the alternating group on $\{M y: y \in G\}$ is of index 2 in $G^{*}$, contrary to hypothesis.

Lemma 1 (Thompson [10]). Suppose that $T$ is a Sylow 2-subgroup of a simple group $G ; \mathrm{SCN}_{3}(T)$ is empty; and $T \supsetneqq D_{8}$. Then $T$ has at most one normal four-group.

Proof. Suppose not, and let $A, B$ be distinct normal four-groups of $T . D=\langle A, B\rangle$ is normal in $T$. If $[A, B]=1$, then $D$ is a normal $E_{8}$ or $E_{16}$ of $T$; therefore $[A, B]$ $=A \cap B$ is of order 2 , and $D \cong D_{8}$.

$T$ stabilizes the two chains $A>A \cap B>1$ and $B>A \cap B>1$, hence stabilizes $D>A \cap B=D^{\prime}>1$. So $T=D \circ C_{T}(D)$; write $C=C_{T}(D)$. $C_{T}(D)$ can have no normal four-group $R$ since then $R A$ and $R B$ contain normal $E_{8}$ 's of $T$. Therefore by Blackburn [3, Theorem 1.1, p. 3], $C$ is cyclic or of maximal class; also, $|C|>2$ since $T \not D_{8}$. A 2-group of maximal class contains a cyclic subgroup of index 2 (Blackburn [2, Theorem 3.4, p. 68]), and is therefore dihedral, semidihedral, or generalized quaternion; in any case, $\Phi(C)$ is cyclic. $\Phi(T)=\Phi(C D)=\Phi(C) \Phi(D)$ (since $C$ and $D$ centralize each other) $=\Phi(C)$; therefore, the involution $z$ of $D^{\prime}=C \cap D$ is the only involution of $T$ which is a square in $T$.

(i) If $t$ is an involution, other than $z$, of $T$, then $C_{T}(t)$ contains $z$ as a square.

Proof. The involutions of $T$ other than $z$ are:

1. Involutions of $D$; for $t \in D, C_{T}(t) \supseteq C$ and $C$ contain $z$ as a square.

2. Involutions of $C$; their centralizers contain $D$ and so contain $z$ as a square.

3. Cross-involutions, namely $c d$ where $c \in C-\langle z\rangle, d \in D-\langle z\rangle$, and either $c^{2}=d^{2}=1$ or $c^{2}=d^{2}=z$. If $c^{2}=d^{2}=z$ then $C_{T}(c d)$ contains $c$ and $d$, hence contains $z$ as a square. If $c^{2}=d^{2}=1$, then $C$ must be dihedral or semidihedral; write

$$
C=\left\langle y, g: y^{2}=1, g^{2^{a}}=1, y^{-1} g y=g^{-1} \text { respectively } g^{-1+2^{a-1}}\right\rangle
$$

where $a \geqq 3$ since $C$ has no normal four-group. Then $c \in y\langle g\rangle$ if $C$ is dihedral; $c \in y\left\langle g^{2}\right\rangle$ if $C$ is semidihedral. We may alter the choice of notation in $C$ so that $c=y$. Then $c d$ is centralized by $g^{2^{a-2}} f$ where $f$ is an element of order 4 in $D$ (since $d \notin\langle z\rangle$ ). So $C_{T}(c d)$ contains $z, g^{2^{a-2}} f$, and $d$; now $\left\langle z, g^{2^{a-2}} f\right\rangle$ is a four-group, and $\left[g^{2^{a-2}} f, d\right]=[f, d]=z$; hence $\left\langle z, g^{2^{a-2}} f, d\right\rangle \cong D_{8}$ with $\langle z\rangle$ as derived group and so is square.

(ii) If $t \neq z$ is an involution of $T$ and $C_{T}(t)$ contains $z$ as a square, then $t$ is not fused to $z$ in $G$.

Proof. If $t$ is fused to $z$ in $G$, then there is $h \in G$ such that $t^{h}=z$ and $C_{T}(t)^{h} \subseteq T$ (by Sylow's theorem in $\left.C_{G}(z)\right) . C_{T}(t)^{h}$ is a subgroup of $T$ which contains $z^{h}$ as a square; therefore $z^{h}=z$; but this contradicts $t^{h}=z$. 
By (i) and (ii), $z$ is not fused in $G$ to any involution of $T$ other than $z$ itself. But then $G$ cannot be simple, by a theorem of Glauberman (Glauberman [7, Theorem 1]; we shall refer to this theorem as "Glauberman's theorem"). This contradiction proves Lemma 1.

Aut $\left(Z_{4} \times Z_{4}\right)$. Aut $\left(Z_{4} \times Z_{4}\right)$ is of order 3.32 and contains a characteristic subgroup $\mathfrak{B}^{+}$which is the set of automorphisms fixing $\Omega_{1}\left(Z_{4} \times Z_{4}\right)$ (elementwise) and is also the stability group of the chain $Z_{4} \times Z_{4}>\Omega_{1}\left(Z_{4} \times Z_{4}\right)>1 . \mathfrak{B}^{+} \cong E_{16}$ and Aut $\left(Z_{4} \times Z_{4}\right) / \mathfrak{B}^{+} \cong \Sigma_{3}$. So Aut $\left(Z_{4} \times Z_{4}\right)$ has a subgroup $\mathcal{S}$ of order $3 \cdot 16$ and index 2 which contains all the Sylow 3-subgroups of Aut $\left(Z_{4} \times Z_{4}\right)$. These Sylow 3subgroups are therefore conjugate to each other by elements of $\mathfrak{B}^{+}$. Therefore all the Sylow 3-subgroups of Aut $\left(Z_{4} \times Z_{4}\right)$ have the same effect on $\mathfrak{B}^{+}$by conjugation. If $\sigma$ is any 3-automorphism of $Z_{4} \times Z_{4}$, then $\mathfrak{B}^{+}=C_{\mathfrak{B}}+(\sigma) \times\left[\mathfrak{B}^{+}, \sigma\right]$ where $C_{\mathfrak{B}}+(\sigma)$ and $\left[\mathfrak{B}^{+}, \sigma\right]$ are both four-groups.

$C_{\mathfrak{B}^{+}}(\sigma)$ and $\left[\mathfrak{B}^{+}, \sigma\right]$ are characteristic subgroups of Aut $\left(\boldsymbol{Z}_{4} \times \boldsymbol{Z}_{4}\right)$, so consist of the same matrices (with integers $\bmod 4$ as coefficients) no matter what basis is chosen for $\boldsymbol{Z}_{4} \times \boldsymbol{Z}_{4}$. These matrices are:

$$
\begin{aligned}
& C_{\mathfrak{B}+(\sigma)}:\left(\begin{array}{ll}
1 & 0 \\
0 & 1
\end{array}\right),\left(\begin{array}{ll}
3 & 0 \\
0 & 3
\end{array}\right),\left(\begin{array}{ll}
1 & 2 \\
2 & 3
\end{array}\right),\left(\begin{array}{ll}
3 & 2 \\
2 & 1
\end{array}\right) . \\
& {\left[\mathfrak{B}^{+}, \sigma\right]:\left(\begin{array}{ll}
1 & 0 \\
0 & 1
\end{array}\right),\left(\begin{array}{ll}
3 & 0 \\
2 & 3
\end{array}\right),\left(\begin{array}{ll}
3 & 2 \\
0 & 3
\end{array}\right),\left(\begin{array}{ll}
1 & 2 \\
2 & 1
\end{array}\right) .}
\end{aligned}
$$

We write $Z$ for the automorphism of $Z_{4} \times Z_{4}$ which inverts every element. Aut $\left(Z_{4} \times Z_{4}\right)$ has three Sylow 2-subgroups, which in matrix form are

$$
\left\langle\left(\begin{array}{ll}
1 & 1 \\
0 & 1
\end{array}\right), \mathfrak{B}^{+}\right\rangle, \quad\left\langle\left(\begin{array}{ll}
1 & 0 \\
1 & 1
\end{array}\right), \mathfrak{B}^{+}\right\rangle, \quad \text { and } \quad\left\langle\left(\begin{array}{ll}
0 & 1 \\
1 & 0
\end{array}\right), \mathfrak{B}^{+}\right\rangle .
$$

All three of these Sylow 2-subgroups have the same action on $C_{\mathfrak{B}^{+}}(\sigma)$ (they centralize $Z=\left(\begin{array}{ll}3 & 0 \\ 0 & 3\end{array}\right)$ and exchange $\left(\begin{array}{ll}1 & 2 \\ 2 & 3\end{array}\right)$ and $\left.\left(\begin{array}{ll}3 & 2 \\ 2 & 1\end{array}\right)\right)$; but they all have different actions on $\left[\mathfrak{B}^{+}, \sigma\right]$ (they each centralize a different nonidentity element).

For reference, we compute the action of $\left(\begin{array}{ll}0 & 1 \\ 1 & 0\end{array}\right)$ on $\mathfrak{B}^{+}$:

$$
\begin{aligned}
\left(\begin{array}{ll}
0 & 1 \\
1 & 0
\end{array}\right): Z & \rightarrow Z \\
\left(\begin{array}{ll}
1 & 2 \\
0 & 1
\end{array}\right) & \rightarrow\left(\begin{array}{ll}
1 & 0 \\
2 & 1
\end{array}\right) \\
\left(\begin{array}{ll}
1 & 0 \\
2 & 1
\end{array}\right) & \rightarrow\left(\begin{array}{ll}
1 & 2 \\
0 & 1
\end{array}\right) \\
\left(\begin{array}{ll}
1 & 0 \\
0 & 3
\end{array}\right) & \rightarrow Z\left(\begin{array}{ll}
1 & 0 \\
0 & 3
\end{array}\right)=\left(\begin{array}{ll}
3 & 0 \\
0 & 1
\end{array}\right) .
\end{aligned}
$$


Thus

$$
C_{\mathfrak{B}^{+}}\left(\begin{array}{ll}
0 & 1 \\
1 & 0
\end{array}\right)=\left[\mathfrak{B}^{+},\left(\begin{array}{ll}
0 & 1 \\
1 & 0
\end{array}\right)\right]=\left\langle Z,\left(\begin{array}{ll}
1 & 2 \\
2 & 1
\end{array}\right)\right\rangle .
$$

3. The case where $\left[\left(N_{G}(T): C_{G}(T)\right]\right.$ has odd prime divisors.

THEOREM 1. Suppose that $T$ is a Sylow 2-subgroup of a simple group $G ; \operatorname{SCN}_{3}(T)$ is empty but $\mathrm{SCN}_{2}(T)$ is not empty; and $T \not D_{8}$. Then (Lemma 1) $T$ has exactly one normal four-group, say $W$.

Suppose also that some odd prime divides $\left[N_{G}(T): C_{G}(T)\right]$.

Then 3 divides $\left[N_{G}(T): C_{G}(T)\right]$, and one of the following holds:

Case 1.1. $A_{G}(T)$ contains a subgroup of order 3 which centralizes $W$. Then $\left[\left(N_{G} T\right): T C_{G}(T)\right]=3 ; T$ is isomorphic to the following group of order $2^{7}$; and $G$ has at most two classes of involutions, represented by $z_{1}$ and iv:

$$
\begin{gathered}
W=\left\langle z_{1}, z_{2}\right\rangle \\
C_{T}(W)=\left\langle W, i, j, k, t, u, v:\langle i, j, k\rangle \cong Q_{8} \text { with } z_{1}\right. \text { as square, } \\
\text { and }\langle t, u, v\rangle \cong Q_{8} \text { with } z_{1} z_{2} \text { as square; } \\
{[i, t]=1,[j, t]=z_{2},[k, t]=z_{2} ;} \\
{[i, u]=z_{2},[j, u]=1,[k, u]=z_{2} ;} \\
\left.[i, v]=z_{2},[j, v]=z_{2},[k, v]=1\right\rangle . \\
T=\left\langle C_{T}(W), \tau: \tau^{2}=1 ; z_{1}^{\tau}=z_{1}, z_{2}^{\tau}=z_{2} z_{1} ; \tau\right. \text { centralizes } \\
\left.i, j, \text { and } k ;[i, \tau]=i z_{2},[u, \tau]=j z_{2},[v, \tau]=k z_{2}\right\rangle .
\end{gathered}
$$

Case 1.2. $A_{G}(T)$ contains a subgroup of order 3 which acts fixed-point-freely on $W$. Then either $T$ is a four-group; or else $\left[N_{G}(T): T C_{G}(T)\right]=3$ or 15 , and $T$ is isomorphic to the following special group of order $2^{6}$ with exactly three involutions:

$A=\langle a\rangle \times\langle b\rangle \cong Z_{4} \times Z_{4}$.

$T=\left\langle A, e, f, h:\right.$ e has matrix $\left(\begin{array}{ll}3 & 0 \\ 2 & 3\end{array}\right)$ on $A$

$$
\begin{aligned}
& \text { f has matrix }\left(\begin{array}{ll}
3 & 2 \\
0 & 3
\end{array}\right) \text { on } A \\
& \text { h has matrix }\left(\begin{array}{ll}
1 & 2 \\
2 & 1
\end{array}\right) \text { on } A
\end{aligned}
$$

with respect to the basis $\{a, b\}$ of $A ; e^{2}=b^{2}, f^{2}=a^{2} b^{2}$,

$$
\left.h^{2}=a^{2} ; e, f, h \text { centralizes each other } h=e^{-1} f^{-1}=e f a^{2}\right\rangle \text {. }
$$

Proof of Theorem 1. By the Schur-Zassenhaus theorem, $N_{G}(T)=T S$ for some subgroup $S$ of odd order. Then $S / C_{S}(T)$ acts faithfully on $T / \Phi(T) . T / \Phi(T)$ is of 
rank at most 4 by the four generator theorem. So $S / C_{S}(T) \hookrightarrow G L_{4}(2)$ and the only odd prime divisors of $\left[N_{G}(T): C_{G}(T)\right]$ are $\in\{3,5,7\}$.

(i) If $\theta$ is an automorphism of $T$ of odd order, $\theta$ centralizes every normal Abelian subgroup of $T$ which it normalizes, if and only if $\theta$ centralizes $W$.

Proof. Suppose $\theta$ centralizes $W$; let $A$ be a normal Abelian subgroup of $T$ normalized by $\theta$. $A$ is cyclic or of rank 2. If cyclic, then Aut $(A)$ is a 2-group, so $\theta$ centralizes $A$. If of rank 2 , then $\Omega_{1}(A) \triangleleft T$, so $\Omega_{1}(A)=W$ by Lemma 1 . If $A$ is not homocyclic, Aut $(A)$ is a 2-group; for, write $A=\langle a\rangle \times\langle b\rangle$ where $a$ is of order $2^{r}$ and $b$ of order $2^{s}<2^{r}$. Then $A / \Phi(A)$ is a four-group whose nonidentity elements are $a \Phi(A), a b \Phi(A)$, and $b \Phi(A)$; of these cosets, the first two consist of elements of order $2^{r}$ while $b \Phi(A)$ consists of elements of order $\leqq 2^{r-1}$, so the only action Aut $(A)$ can have on $A / \Phi(A)$ is to exchange $a \Phi(A)$ and $a b \Phi(A)$. So [Aut $(A)$ : Aut $\left._{0}(A)\right] \leqq 2$, where $\operatorname{Aut}_{0}(A)$ is the set of automorphisms of $A$ which act trivially on $A / \Phi(A)$. Aut $_{0}(A)$ is a 2-group, so Aut $(A)$ is a 2-group.

So we may assume $A$ is homocyclic of rank 2. Then Aut $(A) / \operatorname{Aut}_{0}(A) \cong \Sigma_{3}$, and Aut $(A) /$ Aut $_{0}(A)$ induces $\Sigma_{3}$ on each subquotient $\Omega_{i}(A) / \Omega_{i-1}(A)$, in particular on $W$. So the only nonidentity odd-order automorphisms of $A$ are of order 3 and they are fixed-point-free on $W$. Since $\theta$ centralizes $W$, then, $\theta$ centralizes $A$.

Let $q$ be any odd prime divisor of $\left[N_{G}(T): C_{G}(T)\right]$ and let $Q / C_{S}(T)$ be a subgroup of $S / C_{S}(T)$ of order $q$.

(ii) If $Q$ centralizes $W$, then $T_{1}=[T, Q]$ is a special 2-group.

Proof. Let $A$ be a characteristic Abelian subgroup of $T_{1}$. Since $T_{1}$ is normal in $T, A$ is normal in $T$, and $Q$ centralizes $A$ by (i). Also $\left[T_{1}, Q\right]=T_{1}$. Therefore $T_{1}$ is special by a theorem of Thompson (Huppert [9, Satz III. 13.6, p. 352]).

$T_{1} \triangleleft T$, so $\Phi\left(T_{1}\right) \triangleleft T$; hence if $T_{1}$ is special, $\left|\Phi\left(T_{1}\right)\right| \leqq 4$. Also $\left[T_{1}: \Phi\left(T_{1}\right)\right] \leqq 16$ by the four generator theorem. If $m=$ rank of $T_{1} / \Phi\left(T_{1}\right)$, then $m \leqq 4$ but also $e$ divides $m$, where $e$ is the smallest positive integer such that $2^{e}-1$ is divisible by $q$. $T_{1} / \Phi\left(T_{1}\right)$ is fixed-point-free under $Q$.

Suppose $q=7$. Then $Q$ centralizes $W$ and so $T_{1}$ is special by (ii); and $m$ has to be 3. $T_{1} \triangleleft T$ so cannot be elementary of order 8 ; hence $\Phi\left(T_{1}\right)>1$. $\left|\Phi\left(T_{1}\right)\right|=2$ or 4 and so $Q$ centralizes $\Phi\left(T_{1}\right)$. Therefore if $T_{0}$ is a hyperplane of $\Phi\left(T_{1}\right), Q$ acts on $T_{1} / T_{0}$. But $T_{1} / T_{0}$ is a nonelementary group of order 16 , and no such group admits an automorphism of order 7 . This contradiction establishes that 7 does not divide $\left[N_{G}(T): C_{G}(T)\right]$.

Suppose $q=5$. Then $Q$ centralizes $W$ and so $T_{1}$ is special by (ii); and $m$ has to be 4. We observe that $T=T_{1}$. For in any case, $T=C_{T}(Q) T_{1}$; so if $T>T_{1}$, there is $T_{2}>T_{1}$ such that $T_{2}$ admits $T Q$ and $\left[T_{2}: T_{1}\right]=2$.

$$
\begin{aligned}
T_{2} / \Phi\left(T_{1}\right) & =C_{T_{2} / \Phi\left(T_{1}\right)}(Q)\left[T_{2} / \Phi\left(T_{1}\right), Q\right] \\
& =C_{T_{2} / \Phi\left(T_{1}\right)}(Q) T_{1} / \Phi\left(T_{1}\right) .
\end{aligned}
$$

So $T_{2} / \Phi\left(T_{1}\right)=\left\langle t \Phi\left(T_{1}\right), T_{1} / \Phi\left(T_{1}\right)\right\rangle$ where $t \Phi\left(T_{1}\right)$ is fixed by $Q$ and $T_{1} / \Phi\left(T_{1}\right)$ is irreducible under $Q$, and $t^{2} \in \Phi\left(T_{1}\right) .\left[T_{1} / \Phi\left(T_{1}\right), t\right]$ is a proper $Q$-subgroup of 
$T_{1} / \Phi\left(T_{1}\right)$, so is 1 . But then $T_{2} / \Phi\left(T_{1}\right)$ is elementary of rank 5 , contradicting the four generator theorem.

Therefore $T=T_{1}$ is special; $|\Phi(T)|=2$ or 4 . If 2 , then $T \cong Q_{8} \circ Q_{8}$ or $Q_{8} \circ D_{8}$. $Q_{8} \circ Q_{8}$ admits no automorphism of order 5, so $T \cong Q_{8} \circ D_{8}$; but $Q_{8} \circ D_{8}$ has more than one normal four-group, contrary to Lemma 1.

So $|\Phi(T)|=4$, and $W=\Phi(T)=Z(T)=T^{\prime}$. W contains all the involutions of $T$ since if $x \in T$ were an involution outside $W$, then $\langle x, W\rangle$ would be a normal $E_{8}$ of $T$. Therefore by Glauberman's theorem, all the involutions of $W$ must be fused together in $G$; and so fused in $N_{G}(T)$ by Burnside's theorem (Huppert [9, Hilfssatz IV.2.5, p. 418]).

So 3 divides $\left[N_{G}(T): C_{G}(T)\right], 7$ does not. Now $\left|G L_{4}(2)\right|=2^{6} \cdot 3^{2} \cdot 5 \cdot 7$; so $S / C_{S}(T) \hookrightarrow G L_{4}(2)$ and is of order $3 \cdot 5$ or $3^{2} \cdot 5$. But $G L_{4}(2)$ contains no subgroups of order $3^{2} \cdot 5$. For a group of this order must be Abelian by Sylow's theorem. Now a Sylow 3-subgroup of $G L_{4}(2)$ is of form $\langle\theta\rangle \times\langle\psi\rangle$ where $\theta$ has a two-dimensional fixed point set and $\psi$ has no nonzero fixed points; $\theta$ cannot be centralized by a 5-element of $G L_{4}(2)$ since 5-elements have no invariant two-dimensional subspaces.

So $\left[S: C_{S}(T)\right]=15$; and $S / C_{S}(T)$ contains a subgroup of order 3 which acts fixed-point-freely on $W$. (This situation will be investigated in greater detail under Case 1.2.)

We now have that 3 must divide $\left[N_{G}(T): C_{G}(T)\right]$ and that $S / C_{S}(T)$ is Abelian of order 3,9 , or 15 .

Case 1.1. $S / C_{S}(T)$ contains a subgroup $Q / C_{S}(T)$ of order 3 which centralizes $W$. $T_{1}=[T, Q]$ is normal in $T Q$. For $x \in S, T_{1}^{x}=[T, Q]^{x}=\left[T^{x}, Q^{x}\right]=[T, Q]$ (since $S / C_{S}(T)$ is Abelian) $=T_{1}$. So $N_{G}(T)=T S$ normalizes $T_{1}$.

(iii) No element of $Z(T) \cap T_{1}$ can be fused in $G$ to a central element of $T$ which lies outside $T_{1}$.

Proof. Fusion within $Z(T)$ is all accomplished in $N_{G}(T)$ by Burnside's theorem; and $N_{G}(T)$ cannot conjugate an element of $T_{1}$ to an element outside $T_{1}$.

$T_{1}$ is special, and $m=\operatorname{rank} T_{1} / \Phi\left(T_{1}\right)$ is 2 or 4 .

(iv) If $m=2$ then $T$ cannot be a Sylow 2-subgroup of a simple group $G$.

Proof. Suppose $m=2$. If $T_{1}$ is Abelian then $T_{1}$ is a normal four-group of $T$. $T_{1} \neq W$ since $T_{1}$ is fixed-point-free under $Q$ and $W$ is centralized by $Q$. But $T$ has only one normal four-group by Lemma 1.

So $T_{1}$ is non-Abelian with $\left[T_{1}: T_{1}^{\prime}\right]=4$. But $T_{1}$ is also special, so $T_{1}^{\prime}=Z\left(T_{1}\right)$ $=\Phi\left(T_{1}\right)$ is elementary. So if $T_{1}=\langle x, y\rangle$ (for $x$ and $y$ independent mod $\Phi\left(T_{1}\right)$ ), then $T_{1}^{\prime}=\langle[x, y]\rangle$ so is of order 2. So $\left|T_{1}\right|=8$ and $T_{1} \cong D_{8}$ or $Q_{8}$. $T_{1}$ admits a nontrivial 3-automorphism, so $T_{1} \cong Q_{8}$.

The central involution $z$ of $T_{1}$ is central in $T$. By Glauberman's theorem, $z$ is fused in $G$ to some other involution of $T$. So by (iii), $z$ is fused to a noncentral involution of $T$.

$\left[T_{1} /\langle z\rangle, T\right]$ is a proper $Q$-subgroup of $T_{1} /\langle z\rangle$, so is 1 , i.e., $T$ centralizes $T_{1} /\langle z\rangle$. Therefore $T=C \circ T_{1}$, where $C=C_{T}\left(T_{1}\right)$, and $\operatorname{SCN}_{3}(T)$ empty $\Rightarrow \operatorname{SCN}_{3}(C)$ empty. 
The noncentral involutions of $T$ are of two forms: (1) the noncentral involutions of $C$ (these are centralized by $T_{1}$ ); (2) $x y$ where $x \in C, x^{2}=z, y \in T_{1}-\langle z\rangle$ (these are centralized by $y$ ). So for every noncentral involution $t$ of $T, C_{T}(t)$ contains $z$ as a square.

Case 1. Every element of $\mathrm{SCN}(C)$ is cyclic (i.e., $C$ has no normal four-group). Then $C$ is cyclic or of maximal class (Blackburn [3, Theorem 1.1, p. 3]). If of maximal class, $C$ is dihedral, semidihedral, or generalized quaternion (as in proof of Lemma 1). $z$ is the only involution of $T$ which is a square in $T$, since $\Phi(T)$ $=\Phi(C) \Phi\left(T_{1}\right)=\Phi(C)$ is cyclic.

Some involution $t \neq z$ of $T$ is fused to $z$ in $G$; there is $g \in G$ with $t^{g}=z$ and $C_{T}(t)^{g} \subseteq T . C_{T}(t)^{g}$ must be a subgroup of $T$ which contains $t^{g}=z$ as a nonsquare and $z^{g}$ as a square; but then $z^{g}$ must be $z$, which contradicts $t^{g}=z$.

Case 2. Some $B \in \mathrm{SCN}(C)$ is not cyclic.

1.2.1. If $H$ is any normal Abelian rank 2 subgroup of $C$, then $z$ is not a square in $H$.

Proof. There is $y \in T_{1}$ with $y^{2}=z$. Suppose $h \in H$ has $h^{2}=z .\langle y, H\rangle$ is a normal Abelian subgroup of $T=C \circ T_{1}$. But $\langle y, H\rangle$ contains the three involutions of $H$ and also the involution $y h$; so $\Omega_{1}(\langle y, H\rangle)$ is of order $\geqq 8$, contradicting $\operatorname{SCN}_{3}(T)$ empty.

$z \in B$, but $z$ is not a square in $B(1.2 .1) . B=\langle b\rangle \times\langle z\rangle$ where $b$ is of order $2^{a}$ $(a \geqq 1) . \Omega_{1}(B)=W$.

If $a=1$ then $B \in \mathrm{SCN}(C) \Rightarrow C=B$ or $C \cong D_{8}$. If $C=B$ then $T$ has no noncentral involutions, which is impossible because $z$ must be fused in $G$ to a noncentral involution of $T$. $C \cong D_{8}$ is impossible by Lemma 1 .

If $a \geqq 2$, let $H=\Omega_{2}(B)=\left\langle b^{2^{a-2}}\right\rangle \times\langle z\rangle$.

1.2.2. $B=C_{C}(H)$.

Proof. Immediate if $a=2$, since then $H=B \in \operatorname{SCN}(C)$.

If $a \geqq 3$ and $C_{C}(H)>B$, let $C_{C}(H) \geqq R>B$ where $R \triangleleft T$ and $[R: B]=2$; write $R=\langle r, B\rangle$. Then $r^{2} \in B$, so $r$ induces (by conjugation) an automorphism of order 2 of $B$. Therefore $r^{-1} b r=b w$ or $b^{-1} w$ for some $w \in W$. But if the latter, then $r$ does not centralize $H$. So $r^{-1} b r=b w$ for some $w \in W$.

$C_{B}(r)=\left\langle b^{2}, W\right\rangle ; r^{2} \in C_{B}(r)$. For any integer $i$,

$$
\left(r b^{i}\right)^{2}=r^{2}(b w)^{i} b^{i}=r^{2} b^{2 i} w^{i} .
$$

So we can choose $r \in R-B$ so that $r^{2} \in W$.

If there is some $r \in R-B$ with $r^{2}=1$, then $\langle r, W\rangle$ is precisely the set of involutions of $R$ and so is a normal $E_{8}$ of $T$, contradicting $\operatorname{SCN}_{3}(T)$ empty.

So $R-B$ contains no involutions, but there is $r \in R-B$ with $r^{2} \in W$ but $r^{2} \neq 1$, and $\langle r, H\rangle$ is precisely the set of elements of order $\leqq 4$ in $R$. So $\langle r, H\rangle \triangleleft T$. $\langle r, H\rangle$ is Abelian of order 16 and exponent 4 and contains only three involutions, so every involution-including $z$ - of $\langle r, H\rangle$ is a square in $\langle r, H\rangle$, contradictory to 1.2.1. 
Since $a \geqq 2, \mho^{a-1}(B)$ consists of a single involution $\neq z$. $\mho^{a-1}(B) \triangleleft T$ and $z$ is central in $T$; so $W$ is central in $T$. So $T$ stabilizes the two chains $H>W>1$ and $\left.T_{1}\right\rangle\langle z\rangle>1$.

Since the stability groups of these chains are elementary, $\Phi(T) \subseteq C_{T}\left(T_{1}\right) \cap C_{T}(H)$ $=C_{C}(H)=B$ by 1.2.2. Therefore, no involution of $T-B$ is a square in $T$.

The involutions of $B$ (being just those of $W$ ) are central in $T$. So some involution $t$ of $T-B$ must be fused to $z$ in $G$; there is $g \in G$ with $t^{g}=z$ and $C_{T}(t)^{g} \subseteq T$. $C_{T}(t)^{g}$ must be a subgroup of $T$ which contains $t^{g}=z$ as a nonsquare and $z^{g}$ as a square. The only involutions of $T$ which could be squares in $T$ are central, and we have seen that $z$ is fused to no central involution of $T$ other than itself; so $z^{g}=z$, which contradicts $t^{g}=z$.

By (iv), $T_{1} / \Phi\left(T_{1}\right)$ is of rank 4. Since $T_{1} \triangleleft T, \Phi\left(T_{1}\right) \neq 1$. The $Q$-module $V=T_{1} / \Phi\left(T_{1}\right)$ is fixed-point-free under $Q$, and its fifteen nonidentity elements fall into five $Q$-orbits of length 3 , each of which (together with 1) constitutes an irreducible $Q$-submodule of $V$. Moreover, if $V_{1}, V_{2}$ are any two irreducible $Q$ submodules of $V$, with nonidentity elements $x_{1}, y_{1}, z_{1}$ respectively $x_{2}, y_{2}, z_{2}$ such that the two sets $x_{i}, y_{i}, z_{i}(i=1,2)$ are cycled in the same order by a given generator of $Q / C_{S}(T)$, then all five irreducible $Q$-submodules of $V$ are exhibited as columns in the following chart:

$$
\begin{array}{ccccc}
x_{1} & x_{2} & x_{1} x_{2} & x_{1} z_{2} & x_{1} y_{2} \\
y_{1} & y_{2} & y_{1} y_{2} & y_{1} x_{2} & y_{1} z_{2} \\
z_{1} & z_{2} & z_{1} z_{2} & z_{1} y_{2} & z_{1} x_{2} .
\end{array}
$$

Moreover, if we choose a generator $a C_{S}(T)$ for $Q / C_{S}(T)$, and choose $x_{i}, y_{i}, z_{i}$ so that $x_{i}^{a}=y_{i}, y_{i}^{a}=z_{i}, z_{i}^{a}=x_{i}(i=1,2)$, then $a$ transforms each element in the chart (v) to the one below it.

$$
T=C_{T}(Q) T_{1} \quad \text { and } \quad C_{T}(Q) \cap T_{1} \subseteq \Phi\left(T_{1}\right) .
$$

(vi) $C_{T}(V)=T_{1}$.

Proof. If not, there is $R$ with $T \geqq R>T_{1},\left[R: T_{1}\right]=2, R=\left\langle r, T_{1}\right\rangle$ for $r \in C_{T}(Q)$, and $R \subseteq C_{T}(V) . R / \Phi\left(T_{1}\right)$ contains a central subgroup of index 2 and so is Abelian. $r^{2} \in C_{T}(Q) \cap T_{1} \subseteq \Phi\left(T_{1}\right)$, so $R / \Phi\left(T_{1}\right)$ is elementary of rank 5 , contradicting the four-generator theorem.

In fact, $C_{T}(Q) \cap T_{1}=\Phi\left(T_{1}\right)$. For if $\left|\Phi\left(T_{1}\right)\right|=2$, then $Q$ centralizes $\Phi\left(T_{1}\right)$ since it normalizes $\Phi\left(T_{1}\right)$; and if $\left|\Phi\left(T_{1}\right)\right|=4$ then $\Phi\left(T_{1}\right)=W$ by Lemma 1 , so $Q$ centralizes $\Phi\left(T_{1}\right)$ by hypothesis on $Q$. Thus $T / \Phi\left(T_{1}\right)$ is a split extension of $V$ by $C_{T}(Q) / \Phi\left(T_{1}\right)$; and the action of $T / T_{1}$ on $V$ is the same as that of $C_{T}(Q) / \Phi\left(T_{1}\right)$.

So the action of $T / T_{1}$ on $V$ centralizes that of $Q / C_{S}(T)$. Therefore each $t \in T$ permutes the five irreducible $Q$-submodules of $V$, and centraliztes any which it normalizes.

(vii) If $T>T_{1}$, then $T$ normalizes exactly one of the irreducible $Q$-submodules of 
$V$, say $E / \Phi\left(T_{1}\right)$. Moreover, for every $t \in T-T_{1}, E / \Phi\left(T_{1}\right)$ is the only irreducible $Q$-submodule normalized by $t$.

Proof. There are five irreducible $Q$-submodules, so at least one-say $V_{1}$-is sent to itself by $T$. If $t \in T-T_{1}$ normalized $V_{1}$ and also another irreducible $Q$-submodule $V_{2}$, then $t$ would centralize $V_{1}$ and $V_{2}$, hence would centralize $V_{1} \times V_{2}=V$, contradicting (vi).

(viii) If $U$ is an irreducible $Q$-submodule of $V$, and $U=K / \Phi\left(T_{1}\right)$, then: if $\left|\Phi\left(T_{1}\right)\right|=2, K$ is $\cong$ either $E_{8}$ or $Q_{8}$; if $\left|\Phi\left(T_{1}\right)\right|=4, K$ is $\cong$ either $E_{16}$ or $Q_{8} \times Z_{2}$.

Proof. If $\left|\Phi\left(T_{1}\right)\right|=2$, then $|K|=8$. The only Abelian group of order 8 having an elementary quotient admitting a fixed-point-free automorphism of order 3 , is $E_{8}$; the only non-Abelian one is $Q_{8}$.

If $\left|\Phi\left(T_{1}\right)\right|=4$, then $\Phi\left(T_{1}\right)=W .|K|=16$ and $W$ is central in $K$, and $K / W$ is a fourgroup.

If $K$ is Abelian, $K \cong E_{16}$. For $K$ is of exponent at most 4. Now the order of $x \in K$ depends only on $x W$ since $W$ is central and elementary in $K$. So if there is $x \in K$ of order 4, then (via $Q$ ) every coset of $W$ in $K$ (other than $W$ itself) consists of elements of order 4. So $K \cong Z_{4} \times Z_{4}$. But the 3-automorphisms of this would not centralize $W$.

If $K$ is non-Abelian then $K \cong Q_{8} \times Z_{2}$. For $W=Z(K)$, and $K$ has three maximal subgroups (all Abelian) containing $W$, which are permuted cyclically by $Q$ and are therefore isomorphic. If they are isomorphic to $\boldsymbol{Z}_{4} \times \boldsymbol{Z}_{2}$, we can choose $i, j, k \in K$ which together with $W$ generate these three maximal subgroups, and such that $i^{a}=j, j^{a}=k, k^{a}=i . i^{2}=w \neq 1, w \in W \cdot j^{2}=\left(i^{a}\right)^{2}=\left(i^{2}\right)^{a}=w^{a}=w$ since $Q$ centralizes $W$; similarly $k^{2}=w$. And also $[i, j]=[j, k]=[k, i]=w$, since (for instance)

$$
\begin{aligned}
w=(i j)^{2} \quad(\text { since } i j \equiv k \bmod W) & =i^{2}\left[i, j^{-1}\right] j^{2} \\
& =i^{2} j^{2}[i, j] \text { since } j^{-1} \equiv j \bmod Z(K) \\
& =w w[i, j] \\
& =[i, j] .
\end{aligned}
$$

Therefore $\langle i, j, k, w\rangle \cong Q_{8}$, and $K \cong Q_{8} \times Z_{2}$.

If these three maximal subgroups of $K$ are $\cong E_{8}$, then we can choose involutions $i, j, k$ of $K$ which together with $W$ generate these three maximal subgroups, and such that $i^{a}=j, j^{a}=k, k^{a}=i$. Now $i j \equiv k \bmod W$, so $(i j)^{2}=k^{2}=1$. But since $i$ and $j$ are involutions, $(i j)^{2}=[i, j]$; so $K=\langle i, j, W\rangle$ is Abelian, contrary to assumption.

(ix) $\left|\Phi\left(T_{1}\right)\right|=4$ (and therefore $\Phi\left(T_{1}\right)=W$ ).

Proof. If $\left|\Phi\left(T_{1}\right)\right|=2$, then $E \triangleleft T \Rightarrow E \cong Q_{8}$ by (viii). $T_{1}=E \circ C_{T_{1}}(E) . C_{T_{1}}(E)$ is $Q$-invariant, so by (viii) is $\cong E_{8}$ or $Q_{8}$; but also $C_{T_{1}}(E) \triangleleft T$, so $C_{T_{1}}(E) \cong Q_{8}$; and - $T=T_{1}$ by (vii). But then $T \cong Q_{8} \circ Q_{8}$, which has normal $E_{8}$ 's.

(x) $E \cong Q_{8} \times Z_{2}$, and $C_{T_{1}}(E)=W=\Phi\left(T_{1}\right)$.

Proof. Since $E \triangleleft T, E \cong Q_{8} \times Z_{2}$ by (viii).

$C_{T_{1}}(E) \cap E=Z(E)=W . C_{T_{1}}(E)$ is $Q$-invariant and normal in $T$, so if $C_{T_{1}}(E)>W$ then $T=T_{1}$ by (vii) and $C_{T_{1}}(E) \cong Q_{8} \times Z_{2}$ by (viii). 
Suppose the square of $C_{T_{1}}(E)$ is the same (nonidentity) element of $W$ as the square of $E$. Then take $x_{1}, y_{1}, z_{1} \in E, x_{2}, y_{2}, z_{2} \in C_{T_{1}}(E)$, or order 4 and cyclically permuted by $Q$; and make the chart (v) using the images in $T_{1} / W$ of these $x_{i}, y_{i}, z_{i}$ $(i=1,2)$. Then the first two columns in the chart contain cosets of $W$ which consist of elements of order 4; and the last three columns (the "diagonals") contain cosets of $W$ which consist of involutions. These latter three then come from $E_{16}$ 's of $T_{1}$, the first two from $Q_{8} \times Z_{2}$ 's (by (viii)). $T$ permutes these three $E_{16}$ 's so must normalize one, contradicting $\mathrm{SCN}_{3}(T)$ empty.

Therefore the square of $C_{T_{1}}(E)$ is different from the square of $E$; so $T=T_{1}$ $\cong Q_{8} \times Q_{8}$. But this is impossible by (xi).

(xi) If $T \cong Q_{8} \times Q_{8}$ then $T$ cannot be a Sylow 2-subgroup of a simple group $G$ (Glauberman [7, Corollary 8, p. 419]).

Proof. Write the two $Q_{8}$ 's with generators $i, j, k$ and square $z$, respectively $a, b, c$ and square $x$, so that $T=\langle i, j, k\rangle \times\langle a, b, c\rangle$. Then $z, x$ are each the square of twelve elements of $T$ while $z x$ is the square of thirty-six elements of $T$. So $z x$ is a characteristic involution of $T . z, x$, and $z x$ are the only involutions of $T$, so by Glauberman's theorem, $z x$ is conjugate in $G$ to $z$ or $x$. Hence by Burnside's theorem $z x$ is conjugate in $N_{G}(T)$ to $z$ or $x$, but this is impossible since $z x$ is a characteristic involution of $T$.

(xii) Elements $i, j, k, t, u, v, z_{1}, z_{2} \in T_{1}$ can be chosen so that:

$W=\left\langle z_{1}, z_{2}\right\rangle$.

$E=\left\langle i, j, k, z_{2}\right\rangle$ and $\langle i, j, k\rangle$ is a $Q_{8}$ with $z_{1}$ as square. $i^{a}=j, j^{a}=k, k^{a}=i$.

$\langle t, u, v\rangle$ is a $Q_{8}$ with $z_{1} z_{2}$ as square. $t^{a}=u, u^{a}=v, v^{a}=t$.

$$
\begin{array}{llll}
{[i, t]=1,} & {[j, t]=z_{2},} & & {[k, t]=z_{2} .} \\
{[i, u]=z_{2},} & {[j, u]=1,} & {[k, u]=z_{2} .} \\
{[i, v]=z_{2},} & {[j, v]=z_{2},} & {[k, v]=1 .}
\end{array}
$$

Proof. By $(x), T_{1} / W \hookrightarrow$ the stability group of $E>W>1$. But $T_{1} / W \cong E_{16} \cong$ the stability group of $E>W>1$; so $T_{1} / W$ induces the full stability group. So if $z_{1}$ is the square of $E$ and we choose $i, j, k \in E$ so that $\langle i, j, k\rangle$ is a $Q_{8}$ and $E=\langle i, j, k\rangle$ $\times\left\langle z_{2}\right\rangle$, then there are elements $t, u, v$ of $T_{1}$ such that

$$
\begin{aligned}
& i^{t}=i, \quad j^{t}=j z_{2}, \quad k^{t}=k z_{2}, \\
& i^{u}=i z_{2}, \quad j^{u}=j, \quad k^{u}=k z_{2}, \\
& i^{v}=i z_{2}, \quad j^{v}=j z_{2}, \quad k^{v}=k .
\end{aligned}
$$

(These three automorphisms are the nonidentity elements of a four-group in Aut $E$, namely the subgroup "shearing $\langle i, j, k\rangle$ onto $z_{2}$.")

If $i, j, k \in E$ have been chosen so that $i^{a}=j, j^{a}=k$, and $k^{a}=i$ (the proof of (viii) shows that they can be so chosen), then the relations

$$
[i, t]=1, \quad[j, t]=z_{2}, \quad[k, t]=z_{2}
$$


become, under conjugation by $a$,

$$
\begin{aligned}
& {\left[j, t^{a}\right]=1, \quad\left[k, t^{a}\right]=z_{2}, \quad\left[i, t^{a}\right]=z_{2} ;} \\
& {\left[k, t^{a^{2}}\right]=1, \quad\left[i, t^{a^{2}}\right]=z_{2}, \quad\left[j, t^{a^{2}}\right]=z_{2} \text {. }}
\end{aligned}
$$

Therefore $u \equiv t^{a} \bmod W$ and $v \equiv t^{a^{2}} \bmod W$; and since $W$ is central in $T_{1}$, we can choose $t, u, v$ acting as above on $E$ so that $t^{a}=u, u^{a}=v, v^{a}=t$.

$\langle t, u, v, W\rangle / W$ is one of the five irreducible $Q$-submodules of $V$. The chart (v) for the submodules $\langle i, j, k, W\rangle / W$ and $\langle t, u, v, W\rangle / W$ is

$$
\begin{array}{ccccc}
i & t & i t & i v & i u \\
j & u & j u & j t & j v \\
k & v & k v & k u & k t .
\end{array}
$$

(Note that $\{i, j, k\}$ and $\{t, u, v\}$ are "sharper" sets of generators than were assumed in (v), in the sense that $Q$ permutes $\{i, j, k\}$ and $\{t, u, v\}$ themselves, not just their cosets modulo $W$. This same sharpness with respect to $Q$ holds then for all five columns in the chart.) The inverse image in $T_{1}$ of each submodule is isomorphic to $E_{16}$ or $Q_{8} \times Z_{2}$, by (viii).

$\langle t, u, v, W\rangle \cong Q_{8} \times Z_{2}$. For if $E_{16}$, then by squaring elements in the columns of the chart we see that $\langle t, u, v, W\rangle$ is the only $E_{16}$ of the five; thus $\langle t, u, v, W\rangle \triangleleft T$, contradicting $\operatorname{SCN}_{3}(T)$ empty.

The square in $\langle t, u, v, W\rangle$ is not $z_{1}=$ the square in $\langle i, j, k\rangle$. For if it were, the squares in the five would be: $z_{1}, z_{1}, 1, z_{2}, z_{2}$ respectively; so again one and only one $\cong E_{16}$ and would be normal in $T$.

We now show that the square in $\langle t, u, v, W\rangle$ is not $z_{2}$ (whereupon it must be $z_{1} z_{2}$, and (xii) is proved).

Suppose the square in $\langle t, u, v, W\rangle$ is $z_{2}$. Then $\langle t, u, v\rangle$ centralizes $\langle i t, j u, k v\rangle$ (which shears $\langle i, j, k\rangle$ onto $z_{1} z_{2}$ and has square $z_{1} \dot{z}_{2}$ ), so $T_{1} \cong Q_{8} \times Q_{8} . T>T_{1}$ by (xi). Take new notation in $T_{1}$ as follows: let $\langle a, b, c\rangle,\langle d, e, f\rangle$ be $Q_{8}$ 's of $T_{1}$ with squares $x, y$ respectively, such that

$$
T_{1}=\langle a, b, c\rangle \times\langle d, e, f\rangle,
$$

and the sets $a, b, c$ and $d, e, f$ are cycled in the same order by $Q$. The chart (v) for these generators is

Squares:

$$
\begin{array}{lllll}
a & d & a d & a f & a e \\
b & e & b e & b d & b f \\
c & f & c f & c e & c d
\end{array}
$$$$
\begin{array}{lllll}
x & y & x y & x y & x y .
\end{array}
$$

Now the subgroups $\langle a, b, c, W\rangle$ and $\langle d, e, f, W\rangle$ of $T_{1}$ centralize each other, while the subgroups of $T_{1}$ corresponding to the last three columns of the chart have 
centralizer in $T_{1}$ equal to $W$. Therefore $T$ must act separately on the first two and two of the last three; and so $\left[T: T_{1}\right]=2$ (by (vii)), $T$ exchanges $\langle a, b, c, W\rangle$ and $\langle d, e, f, W\rangle$, and therefore $W$ is not central in $T . T=C_{T}(Q) T_{1}$ and $C_{T}(Q) \cap T_{1}=W$. $\left|C_{T}(Q)\right|=8$ and $C_{T}(Q)$ is non-Abelian (since $W$ is not central in $T$ ), so $C_{T}(Q) \cong D_{8}$ and we can take an involution $\tau \in C_{T}(Q)$ such that $C_{T}(Q)=\langle W, \tau\rangle$. d, e,f can be chosen so that $a^{\tau}=d, b^{\tau}=e, c^{\tau}=f$. Then $C_{V}(\tau) \supseteq\langle a d, b e, c f, W\rangle / W ; C_{V}(\tau)=E / W$ is of order 4 by (vii), so $E=\langle a d, b e, c f, W\rangle$.

We now show that $T-T_{1}=\tau T_{1}$ contains eight involutions, all conjugate in $T$ to $\tau$. The involutions of $\tau T_{1}$ are the elements $\tau k$ where $k \in T_{1}$ and $\tau$ inverts $k . V$ is elementary, so each such $k$ must have $k W \in C_{V}(\tau)$, and the involutions of $\tau T_{1}$ all lie in $\tau\langle a d, b e, c f, W\rangle$.

The involutions of $\tau W$ are $\tau$ and $\tau x y=\tau^{x}$.

$\tau a d W, \tau b e W$, and $\tau c f W$ are sent to one another by $Q$, since $\tau \in C_{T}(Q)$. Also $Q$ normalizes $T$. If all the involutions of $\tau a d W$ are $T$-conjugate to $\tau$, then all the involutions of the $Q$-conjugates of $\tau a d W$ will be $T$-conjugate to $\tau$ (since $\tau \in C_{T}(Q)$ ). Hence all the involutions of $\tau\langle a d, b e, c f, W\rangle$ will be $T$-conjugate to $\tau$.

$\tau a d W=\{\tau a d, \tau a d x y, \tau a d x, \tau a d y\}$. We compute that:

$$
\begin{aligned}
(a d)^{\tau} & =d a=a d \text { since } a \text { centralizes } d . \\
(a d x y)^{\tau} & =a d x y . \\
(a d x)^{\tau} & =d a y ;(a d x)(d a y)=(a d)(d a) x y=1 . \\
(a d y)^{\tau} & =d a x ;(a d y)(\text { dax })=1 .
\end{aligned}
$$

So the involutions of $\tau a d W$ are $\tau a d x$ and $\tau a d y$. Now

$$
\begin{aligned}
\tau^{a} & =\tau\left(\tau a^{-1} \tau\right) a=\tau d^{-1} a=\tau d y a=\tau a d y . \\
\tau^{a x} & =\left(\tau^{a}\right)^{x}=(\tau a d y)^{x}=\tau^{x} a d y=\tau x y a d y=\tau a d x .
\end{aligned}
$$

So the involutions of $\tau a d W$ are $T$-conjugate to $\tau$. Hence the involutions of $\tau T_{1}$ are all conjugate to $\tau$ in $T$.

We now show that this $T$ cannot be a Sylow 2-subgroup of a simple group $G$. The $T$-conjugacy classes of involutions of $T$ are $\{x y\},\{x, y\}$, and the eight involutions of $\tau T_{1}$. By Glauberman's theorem, $x y$ is fused in $G$ to $\tau$ or to $x$.

Suppose $x y$ is fused to $\tau$; then there is $g \in G$ with $\tau^{g}=x y$ and $C_{T}(\tau)^{g} \subseteq T . C_{T}(\tau)$ is of order $|T| / 8=16$, so $\left|C_{T_{1}}(\tau)\right|=8 ; C_{T_{1}}(\tau)=\langle a d, b e, c f, x y\rangle \cong Q_{8}$. Thus $C_{T}(\tau)$ contains $x y$ as a square. So $C_{T}(\tau)^{g} \subseteq T$ contains $(x y)^{g}$ as a square. Now $\Phi(T) \subseteq T_{1}$ and the only involutions of $T_{1}$ are $x, y$ and $x y$; so the only involutions of $T$ which are squares in $T$, are $x, y$, and $x y .(x y)^{g} \neq x y$, so $(x y)^{g}=x$ or $y$.

So if $\tau$ is fused to $x y$, then $x$ is fused to $x y$. So in any case $x$ is fused to $x y$ in $G$, and there is $g \in G$ with $x^{g}=x y$ and $C_{T}(x)^{g}=T_{1}^{g} \subseteq T . T_{1}$ is the only maximal subgroup of $T$ to contain a central four-group in which every element is a square; so $T_{1}^{g}=T_{1}$. But then $x^{g}=x y$ is impossible because $x y$ is a characteristic involution of $T_{1}$. 
(xiii) $T_{1}$ is generated by two $E_{16}$ 's; and $\left[T: T_{1}\right]=2$.

Proof. The chart (v) for the generators $\{i, j, k\}$ and $\{t, u, v\}$ is

Squares: $\quad \begin{array}{llllll}z_{1} & z_{1} z_{2} & z_{2} & 1 & 1 .\end{array}$

So by (viii), the last two submodules come from $E_{16}$ 's of $T_{1} . T$ must act separately on the two sets of submodules other than $E / W$ which come from $Q_{8} \times Z_{2}$ 's respectively $E_{16}$ 's; so $\left[T: T_{1}\right]=2$ by (vii).

(xiv) $T=T_{1} C_{T}(Q)$ where $C_{T}(Q) \cong D_{8}$. We can take an involution $\tau \in C_{T}(Q)-W$ such that

$$
\begin{array}{r}
T=\left\langle i, j, k, t, u, v, z_{1}, z_{2}, \tau: i, j, k, t, u, v, z_{1}, z_{2}\right. \\
\text { satisfy the relations given in (xii); } \\
\begin{array}{r}
z_{1}^{\tau}=z_{1}, z_{2}^{\tau}=z_{1} z_{2} ; \tau^{2}=1 ; \tau \text { centralizes } i, j, \text { and } k ; \\
\left.[t, \tau]=i z_{2},[u, \tau]=j z_{2},[v, \tau]=k z_{2}\right\rangle .
\end{array}
\end{array}
$$

Proof. $T=T_{1} C_{T}(Q)$ and $T_{1} \cap C_{T}(Q)=W$; so $\left|C_{T}(Q)\right|=8$. $T$ must conjugate $\langle t, u, v, W\rangle$ to $\langle i t, j u, k v, W\rangle$, and hence $z_{1} z_{2}$ to $z_{2}$. So $C_{r}(Q) \cong D_{8}$ and there is an involution $\tau \in C_{T}(Q)$ such that $C_{T}(Q)=\langle W, \tau\rangle$.

By (vii), $C_{V}(\tau) \supseteq E / W$. The automorphism $X$ induced on $V$ by $\tau$ is of order 2, so in the ring of endomorphisms of $V, 0=X^{2}-1=(X-1)^{2}$, and so

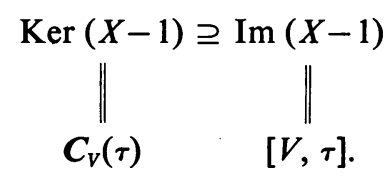

Also $\operatorname{Dim} \operatorname{Ker}(X-1)+\operatorname{Dim} \operatorname{Im}(X-1)=\operatorname{Dim}(V)=4$; so $C_{V}(\tau)=[V, \tau]=E / W$.

Since $\tau \in C_{T}(Q)$ and $\tau$ normalizes $E / W, \tau$ centralizes $E / W$, and so $[i, \tau],[j, \tau]$, and $[k, \tau]$ are all $\in W$. We will now show that $[i, \tau]=[j, \tau]=[k, \tau]=1$. For, writing $[i, \tau]=w \in W$ and conjugating by $a$, we get

$$
w=w^{a}=\left[i^{a}, \tau^{a}\right]=[j, \tau],
$$

and similarly $w=[k, \tau]$. Now either $i j=k$ or $i j=k z_{1}$; since $z_{1} \in Z(T)$, in either case we have $(i j)^{\imath}=(i j) w$. So

$$
(i j) w=(i j)^{\imath}=i^{\imath} j^{\imath}=(i w)(j w)=i j w^{2}=i j .
$$

Hence $w=1$.

We will now normalize $i, j$, and $k$ so that $[t, \tau]=i z_{2},[u, \tau]=j z_{2},[v, \tau]=k z_{2}$. Since $E / W=C_{V}(\tau)=[V, \tau],[t, \tau]=e$ for some $e \in E$. Now $t^{2}=z_{1} z_{2}$; conjugating by $\tau$, we get

$$
z_{2}=\left(t^{2}\right)^{\mathfrak{t}}=\left(t^{\tau}\right)^{2}=(t e)^{2}=t^{2} e^{t} e=z_{1} z_{2} e^{t} e
$$


So $e$ must satisfy $e^{t} e=z_{1}$. The elements of $E$ satisfying this are $\{i w: w \in W\}$. So $t^{\tau}=t i w$ for some $w \in W$.

Now $i^{-1}, j^{-1}, k^{-1}$ are carried to one another by $a$ as are $i, j$, and $k$, and indeed they satisfy the relations of (xii) with respect to $t, u, v, z_{1}, z_{2}$ just as do $i, j$, and $k$. So if necessary we may replace $i, j, k$ by $i^{-1}, j^{-1}, k^{-1}$ to get: $w=1$ or $z_{2}$.

Since $\tau^{2}=1$,

$$
\begin{aligned}
t=t^{\tau^{2}} & =(t i w)^{\tau}=t^{\tau} i^{\tau} w^{\tau} \\
& =(t i w) i w^{\tau}=t z_{1} w w^{\tau} .
\end{aligned}
$$

Therefore $w$ must satisfy $w w^{\tau}=z_{1}$. So $w=z_{2}$, and $[t, \tau]=i z_{2}$. Conjugating by $a$, we get $[t, \tau]^{a}=\left[t^{a}, \tau^{a}\right]=[u, \tau]=j z_{2}$; and $[v, \tau]=k z_{2}$.

So the isomorphism type of $T$ is determined.

$\Phi(T)=E$ is of index 8 in $T$; so $5 \nmid\left[N_{G}(T): C_{G}(T)\right]$ since an automorphism of order 5 of $T$ must act faithfully on $T / \Phi(T)$ and 5 does not divide $\left|G L_{3}(2)\right|$. Therefore $\left[N_{G}(T): T C_{G}(T)\right]=3$, as claimed in the statement of Theorem 1 .

We will now show that $G$ has at most two classes of involutions, represented by $z_{1}$ and $i v$.

The involutions of $T_{1}$ are the nonidentity elements of the two $E_{16}$ 's $X=$ $\langle i v, j t, k u, W\rangle$ and $Y=X^{\imath}=\langle i u, j v, k t, W\rangle$. The involutions of $\tau T_{1}$ are the elements $\tau r$ where $r \in T_{1}$ and $\tau$ inverts $r$. Such $r$ must have $r W \in C_{V}(\tau)=E / W$, hence $r \in E$; so the involutions of $\tau T_{1}$ lie in $\tau E$. The involutions of $\tau W$ are $\tau$ and $\tau z_{1}$. The involutions of $\tau i W=\left\{\tau i, \tau i z_{1}, \tau i z_{2}, \tau i z_{1} z_{2}\right\}$ are $\tau i z_{2}$ and $\tau i z_{1} z_{2}$; conjugating by $a$, we find that $\tau j W$ and $\tau k W$ likewise contain two involutions each. Hence $\tau T_{1}$ contains eight involutions.

Now $\left\langle C_{T_{1}}(\tau), W\right\rangle / W \subseteq C_{V}(\tau)=E / W$, hence $C_{T_{1}}(\tau) \subseteq E$. Computing inside $E$, we get $C_{E}(\tau)=\langle i, j, k\rangle$. So $\left[T_{1}: C_{T_{1}}(\tau)\right]=8$, so there are eight $T_{1}$-conjugates of $\tau$, all lying in $\tau T_{1}$. Hence all the involutions of $\tau T_{1}$ are $T_{1}$-conjugate.

Also, each coset of $W$ in $X$ is a single $T_{1}$-conjugacy class of involutions; for $\left[i v, T_{1}\right]$ contains:

$$
\begin{aligned}
{[i v, i] } & =[v, i]=z_{2}, \\
{[i v, j] } & =[i, j][v, j]=z_{1} z_{2}, \\
{[i v, k] } & =[i, k][v, k]=z_{1}
\end{aligned}
$$

Hence $\left[i v, T_{1}\right]=W$. Conjugating by $a$, we get $\left[j t, T_{1}\right]=W$ and $\left[k u, T_{1}\right]=W$. So $X-W$ is a single class under $T_{1} Q$. Since $X^{\imath}=Y,(X-W) \cup(Y-W)$ is a single class under $T Q$.

So there are four classes of involutions of $T$ under conjugation by $T Q$, namely $\left\{z_{1}\right\} ;\left\{z_{2}, z_{1} z_{2}\right\}$; the involutions of $\tau T_{1}$; and $(X-W) \cup(Y-W)$. In particular, $X$ and $Y$ are the only $E_{16}$ 's of $T$. Also, $\Phi(T)=E$, so the only involutions which are squares in $T$ are the involutions of $W$.

(xv) If $\tau$ and $z_{1}$ are fused in $G$, then $z_{2}$ and $z_{1}$ are fused in $G$. 
Proof. Suppose $\tau$ is $G$-conjugate to $z_{1} . C_{T}(\tau)=\langle\tau, i, j, k\rangle$, so $z_{1}$ is a square in $C_{T}(\tau)$. So if $T^{*}$ is a Sylow 2-subgroup of $C_{G}(\tau)$ (and hence of $G$ ) with $T^{*} \supseteq C_{T}(\tau)$, then $z_{1}$ is a square in $T^{*}$, so $z_{1} \in W^{*}$. Also $\tau \in W^{*}$. So $W^{*}$ contains two different $G$-conjugates of the central involution $\tau$ of $T^{*}$.

(xvi) $\tau$ and $z_{1}$ are fused in $G$ (whereupon by (xv), $G$ has at most two classes of involutions, represented by $z_{1}$ and $i v$ ).

Proof. $\tau$ is fused to some element of $T_{1}$, by Lemma A. If $\tau$ is not fused to $z_{1}$, then one of the following holds:

(xvi.i) $\tau$ is fused to $z_{2}$, and $z_{1}$ is not fused to $z_{2}$ or $\tau$.

(xvi.ii) $\tau$ is not fused to $z_{2}$ but is fused to $i v \in X$, and $z_{1}$ is not fused to $i v$, and $z_{2}$ is not fused to $i v$.

If (xvi.ii) holds, then $C_{T}(i v)=X$ and $C_{T}(\tau)=(\langle i, j, k\rangle \times\langle\tau\rangle)^{g}$ are both Sylow 2-subgroups of $C_{G}(i v)$ (where $\tau^{g}=i v$ ). But this contradicts Sylow's theorem in $C_{G}(i v)$ since $X \nsubseteq Q_{8} \times Z_{2}$.

Therefore (xvi.i) holds. $z_{1}$ is fused to some involution of $T$ other than itself, by Glauberman's theorem; therefore $z_{1}$ is fused to $i v$.

Now $T_{1}, Q \subseteq N_{G}(X)$ and $T_{1}$ is a Sylow 2-subgroup of $N_{G}(X) . C_{T_{1}}(X)=X$ and $\boldsymbol{A}_{T_{1} Q}(X) \cong \Sigma_{4}^{+}$. We will show that $\boldsymbol{A}_{G}(X)$ is transitive on the nonidentity elements of $X$, which contradicts (xvi.i) and so proves (xvi). We already have that $X-W$ is a single class under $T_{1} Q$.

Since $z_{1}$ is fused to $i v$, there is a Sylow 2-subgroup $T^{*}$ of $G$ in which $i v$ is the central involution, such that $T^{*} \supseteq X=C_{T}(i v) . T_{1}^{*}$ is then a Sylow 2-subgroup of $N_{G}(X)$ (since $T_{1}^{*}$ is the normalizer in $T^{*}$ of both of the $E_{16}$ 's of $T^{*}$ ), and there is $y \in N_{G}(X)$ with $\left(T_{1}^{*}\right)^{y}=T_{1}$. Then $\left(W^{*}\right)^{y}=W$, so $(i v)^{y} \in W$. So the set $(X-W)$ $\cup\left\{(i v)^{y}\right\}$ is part of an orbit of $\boldsymbol{A}_{G}(X)$ on the nonidentity elements of $X$. No orbit of $\boldsymbol{A}_{G}(X)$ can have size 13 since 13 does not divide $\left|G L_{4}(2)\right|$. So if $\boldsymbol{A}_{G}(X)$ is not transitive, it must have two orbits of sizes 14 and 1 , the orbit of size 1 being $\subseteq W$; call this orbit $\{z\}$.

If this occurs, we observe that $\boldsymbol{A}_{G}(X)$ is faithfully represented on $X /\langle z\rangle$, i.e., $\boldsymbol{A}_{G}(X)$ intersects the stability group $L$ of the chain $\left.X\right\rangle\langle z\rangle>1$ trivially. For $\boldsymbol{A}_{G}(X) \cap L \triangleleft \boldsymbol{A}_{G}(X)$; and since $\boldsymbol{A}_{T_{1}}(X)$ is a Sylow 2-subgroup of $\boldsymbol{A}_{G}(X)$ and $\left[X, T_{1}\right]$ is all of $W, A_{G}(X) \cap L$ has order 2 (if nontrivial). Then $A_{G}(X) / A_{G}(X) \cap L$ has order twice an odd number, so has a normal 2-complement $N_{1} / A_{G}(X) \cap L$; $N_{1}$ has order twice an odd number so has a normal 2-complement $N . N$ is then a normal 2-complement in $\boldsymbol{A}_{G}(X)$. But $\boldsymbol{A}_{G}(X)$ contains $\boldsymbol{A}_{T_{1} Q}(X)$ which does not have a normal 2-complement; so $\boldsymbol{A}_{G}(X)$ cannot have a normal 2-complement.

So $\boldsymbol{A}_{G}(X)$ has a faithful representation in $G L_{3}(2)$, whose order is $2^{3} \cdot 3 \cdot 7$. Moreover, $\left|A_{G}(X)\right|$ is 12 times an odd number and is divisible by 14 , so is 12.7 ; but this is impossible since $G L_{3}(2)$ has no subgroups of index 2.

Case 1.2. $S / C_{S}(T)$ contains a subgroup $Q / C_{S}(T)$ of order 3 which acts fixed-pointfreely on $W$. Then $W=\Omega_{1}(Z(T))$.

Let $A$ be a subgroup of $T$ with $A \supseteq W, A$ Abelian, $A \triangleleft T Q$, and $A$ maximal 
subject to these conditions. $A$ is of rank 2 and is homocyclic (for we have seen in the proof of (i) in Case 1.1 that a nonhomocyclic group would not admit an automorphism of order 3 ).

If $A=W$, then $T=W$ (so that $T$ is a four-group). For suppose $T>A=W$. TQ acts on $T / W$ and preserves $\Omega_{1}(Z(T / W))=Z / W$ say; moreover, $T$ acts trivially on $Z / W$, so the $T Q$-invariant subgroups of $Z / W$ are just the $Q$-invariant subgroups. Suppose $C_{Z / W}(Q)>1$. Then there is a $T Q$-invariant subgroup $L$ of $T$ with $Z \geqq L>W$, and $[L: W]=2$. But $W$ is central in $T$, so $L$ is Abelian, which contradicts the maximality of $A=W$. Therefore $Z / W$ is fixed-point-free under $Q$ and is a direct product of irreducible $Q$-modules, each of which is a four-group. Let $K / W$ be one of these irreducible $Q$-modules; then $W$ is central in $K$, and $Q$ acts fixed-point-freely on $W$ and on $K / W$. We now show (Lemma 2) that $K$ is Abelian, which again contradicts the maximality of $A=W$.

LEMMA 2. Let $K$ be a group of order 16 , with a central four-subgroup $W$, such that $K$ admits an automorphism $\sigma$ of order 3 which preserves $W$ and acts fixed-pointfreely on $W$ and on $K / W$. Then $K \cong E_{16}$ or $Z_{4} \times Z_{4}$.

Proof. Let $k \in K, k \notin W . k W,(k W)^{\sigma}$, and $(k W)^{\sigma^{2}}$ are the three nonidentity cosets of $W$ in $K . k^{2} \in W$, and since $W$ is elementary and central, $(k W)^{2}=k^{2}$.

If $k^{2}=1$, then $(k W)^{2}=\left((k W)^{\sigma}\right)^{2}=\left((k W)^{\sigma^{2}}\right)^{2}=1$, and so $K$ is of exponent 2 and hence elementary Abelian.

If $k^{2}=w \neq 1$, then $(k W)^{2}=w ;\left((k W)^{\sigma}\right)^{2}=w^{\sigma} ;$ and $\left((k W)^{\sigma^{2}}\right)^{2}=w^{\sigma^{2}}$. So each element of $W$ is a square in $K$.

Now $K=\left\langle k, k^{\sigma}, W\right\rangle$; and

$$
\left[k, k^{\sigma}\right]=k^{-1}\left(k^{\sigma}\right)^{-1} k k^{\sigma}=k w k^{\sigma} w^{\sigma} k k^{\sigma}=w w^{\sigma}\left(k k^{\sigma}\right)^{2} .
$$

But $k k^{\sigma} \equiv k^{\sigma^{2}} \bmod W$, so $\left(k k^{\sigma}\right)^{2}=w^{\sigma^{2}}$; so

$$
\left[k, k^{\sigma}\right]=w w^{\sigma} w^{\sigma^{2}}=1 .
$$

So $K$ is Abelian of exponent 4 and every involution is a square; hence $K \cong Z_{4} \times Z_{4}$.

If $T=A$ then $G$ cannot be simple unless $T$ is a four-group, by a result of $\mathrm{R}$. Brauer [4, Theorem 1, p. 317].

Thus if $T$ is not a four-group, $T>A>W$. Let $B=\Omega_{2}(A) ; B \cong Z_{4} \times Z_{4}$.

(xvii) $A=C_{T}(B)$.

Proof. $C_{T}(B)$ is $Q$-invariant. Also $C_{T}(B)$ is metacyclic (Alperin [1, Corollary, p. 110]) and the only metacyclic 2-group admitting an automorphism of order 3 which acts on $W$ as $Q$ does, is Abelian (since otherwise its derived group is cyclic and $Q$-invariant) and homocyclic; so $C_{T}(B)=A$ by maximality of $A$.

Therefore $T / A \hookrightarrow$ Aut $B$; since $T$ centralizes $W, T / A \rightarrow \mathfrak{B}^{+}$(see Chapter II). $T / A$ is $Q$-invariant, so the image of $T / A$ in $\mathfrak{B}^{+}$either contains $\left[\mathfrak{B}^{+}, \sigma\right]$ or else is contained in $C_{\mathfrak{B}}+(\sigma)$ (where $\sigma$ is any 3-automorphism of $B$ ).

We now show that $A$ must be of exponent 4 . 
(xviii) If $A$ is of exponent $2^{r}$ for $r \geqq 3$, then the subgroup of $\mathfrak{B}^{+}$induced on $B$ by $T / A$ does not contain $\left[\mathfrak{B}^{+}, \sigma\right]$ (and hence lies in $C_{\mathfrak{B}}+(\sigma)$ ).

Proof. Suppose the contrary, and let $T_{1}$ be the inverse image in $T$ of $\left[\mathfrak{B}^{+}, \sigma\right] ; T_{1}$ is fixed-point-free under $Q$ since $A$ and $T_{1} / A$ are.

If $T_{1}-A$ has no involutions then $T_{1}$ is of exponent 4 by a result of $\mathrm{G}$. Higman [8], which contradicts our assumption regarding $A$.

So $T_{1}$ contains an involution $t, t \notin A$. Let $\rho$ be a generator for $A_{Q}(T)$; since $\rho$ is fixed-point-free on $T_{1}$,

$$
t t^{\rho} t^{\rho^{2}}=1, \quad t t^{\rho^{2}} t^{\rho}=1
$$

and therefore $t^{\rho}$ commutes with $t^{\rho^{2}}$; hence also $\left[t^{\rho^{2}}, t\right]=1$ and $\left[t, t^{\rho}\right]=1$, so $t, t^{\rho}$, and $t^{\rho^{2}}$ all centralize each other. $t t^{\rho} t^{\rho^{2}}$ is fixed by $\rho$, so must equal 1 . Also $1, t, t^{\rho}, t^{\rho^{2}}$ form a complete set of coset representatives for $A$ in $T_{1}$. So they constitute a fourgroup $V$ which is a $Q$-complement to $A$ in $T_{1}$.

Choose a basis for $A$, and take the $2^{r-2}$ th powers of the members of this basis as a basis for $B$. We can then write matrices for automorphisms of $A$ and $B$.

$\left[\mathfrak{B}^{+}, \sigma\right]$ contains the matrix $\left(\begin{array}{ll}1 & 2 \\ 2 & 1\end{array}\right)$, so there is $v \in V$ such that $v$ induces $\left(\begin{array}{ll}1 & 2 \\ 2 & 1\end{array}\right)$ on $B$. If $\left(\begin{array}{ll}\alpha & \beta \\ \gamma\end{array}\right)$ is the matrix of $v$ on $A$, we have

$$
\left(\begin{array}{ll}
\alpha & \beta \\
\gamma & \delta
\end{array}\right) \equiv\left(\begin{array}{ll}
1 & 2 \\
2 & 1
\end{array}\right) \bmod 4
$$

Since $v^{2}=1$, we also have

$$
\left(\begin{array}{ll}
\alpha^{2}+\beta \gamma & \beta(\alpha+\delta) \\
\gamma(\alpha+\delta) & \beta \gamma+\delta^{2}
\end{array}\right)=\left(\begin{array}{ll}
\alpha & \beta \\
\gamma & \delta
\end{array}\right)^{2} \equiv\left(\begin{array}{ll}
1 & 0 \\
0 & 1
\end{array}\right) \bmod 2^{r}
$$

$\beta$ and $\gamma$ are each twice an odd number by $\left({ }^{*}\right)$, so $(\alpha+\delta)$ is a multiple of $2^{r-1}$ by $\left({ }^{* *}\right)$. Also $\alpha^{2}=\delta^{2}$ by $\left({ }^{* *}\right)$, i.e. $\left(\alpha \delta^{-1}\right)^{2}=1$. $(\alpha$ and $\delta$ are odd and therefore invertible $\bmod 2^{r}$.) Therefore $\alpha \delta^{-1}= \pm 1+i 2^{r-1}$ for $i=0$ or 1 ; i.e.,

$$
\alpha=\delta\left( \pm 1+i 2^{r-1}\right) \text {. }
$$

Therefore $\alpha+\delta=\delta\left( \pm 1+1+i 2^{r-1}\right)$ is $\equiv 0 \bmod 2^{r-1}$, so -1 must occur, i.e. $\alpha=-\delta+i 2^{r-1}$. But then $\alpha \equiv-\delta \bmod 4$, whereas $\left(^{*}\right)$ implies that $\alpha \equiv \delta \bmod 4$. This contradiction establishes (xviii).

(xix) If $A$ is of exponent $2^{r}$ for $r \geqq 3$, then $[T: A]=2$, and for suitable choice of basis in $A$,

$$
T=\left\langle A, t: t^{2}=1 \text { and the matrix of } t \text { on } A \text { is }\left(\begin{array}{cc}
-1+i 2^{r-1} & j 2^{r-1} \\
j 2^{r-1} & -1+(i+j) 2^{r-1}
\end{array}\right)\right\rangle
$$

for some $i, j=0$ or 1 .

Proof. By (xviii), the subgroup of $\mathfrak{B}^{+}$induced on $B$ by $T / A$ lies in $C_{\mathfrak{B}}+(\sigma)$, and so $[T, Q]=A$. $T=[T, Q] C_{T}(Q)$ splits $T$ over $A$, with $C_{T}(Q)$ elementary of order 2 or 4 . 
All the elements of order 3 in Aut $(A)$ are conjugate in Aut $(A)$. So if $\rho$ is a chosen generator for $A_{Q}(T)$, we can take any 3-matrix we like to represent the action of $\rho$ on $A$, by choosing basis suitably in $A$. Choose the matrix $\left(\begin{array}{rr}0 & -1 \\ -1 & -1\end{array}\right)$ for $\rho$. Then the automorphisms of $A$ induced by elements of $C_{T}(Q)$ have matrices $\left(\begin{array}{ll}\alpha & \beta \\ \gamma & \delta\end{array}\right)$ which must centralize $\left(\begin{array}{rr}0 & -1 \\ -1 & -1\end{array}\right)$-this is equivalent to $\delta=\alpha-\beta$ and $\gamma=-\beta$.

Since $C_{T}(Q)$ is elementary, we must have $\left(\bmod 2^{r}\right)$

$$
\left(\begin{array}{ll}
1 & 0 \\
0 & 1
\end{array}\right) \equiv\left(\begin{array}{cc}
\alpha & \beta \\
-\beta & \alpha-\beta
\end{array}\right)^{2}=\left(\begin{array}{cc}
\alpha^{2}-\beta^{2} & 2 \alpha \beta-\beta^{2} \\
-2 \alpha \beta+\beta^{2} & \alpha^{2}-2 \alpha \beta
\end{array}\right)
$$

or equivalently

$$
\alpha^{2}-\beta^{2} \equiv 1 \bmod 2^{r}, \quad 2 \alpha \beta \equiv \beta^{2} \bmod 2^{r} .
$$

$\beta$ cannot be odd. For if it were, we could cancel it in $2 \alpha \beta \equiv \beta^{2}$, getting $2 \alpha \equiv \beta$, so $\beta$ is even.

So $\beta$ is even; then $\alpha$ must be odd since $\left(\begin{array}{cc}\alpha & \alpha \beta \\ -\beta & \alpha-\beta\end{array}\right)$ is nonsingular. Since $2 \alpha \beta \equiv \beta^{2}$, either $\beta$ is twice an odd number or $2 \alpha \beta \equiv \beta^{2} \equiv 0 \bmod 2^{r}$.

If $\beta$ is twice an odd number-say $\beta=2 \beta_{0}$ for odd $\beta_{0}$ the equation $2 \alpha \beta \equiv \beta^{2} \bmod 2^{r}$ becomes

$$
\begin{aligned}
& 2 \alpha 2 \beta_{0} \equiv 4 \beta_{0}^{2} \quad \bmod 2^{r} \\
& \Leftrightarrow 4 \alpha \equiv 4 \beta_{0} \quad \bmod 2^{r} \\
& \Leftrightarrow \beta_{0} \equiv \alpha+k 2^{r-2} \quad \text { for some integer } k .
\end{aligned}
$$

Then the equation $\alpha^{2}-\beta^{2} \equiv 1 \bmod 2^{r}$ becomes

$$
\alpha^{2}-\left(2 \alpha+k 2^{r-1}\right)^{2} \equiv 1 \bmod 2^{r} \Leftrightarrow-3 \alpha^{2} \equiv 1 \bmod 2^{r} .
$$

In particular, $-3 \alpha^{2} \equiv 1 \bmod 8$. But this is impossible since the square of any odd number is $\equiv 1 \bmod 8$.

So $2 \alpha \beta \equiv \beta^{2} \equiv 0 \bmod 2^{r}$. Therefore $\beta=j 2^{r-1}(j=0$ or 1$) . \alpha^{2}-\beta^{2} \equiv 1 \bmod 2^{r}$ becomes $\alpha^{2} \equiv 1 \bmod 2^{r}$, so $\alpha= \pm 1+i 2^{r-1}(i=0$ or 1$)$. If $\alpha=1+i 2^{r-1}$ then $\left(\begin{array}{cc}\alpha & \alpha \beta \\ -\beta & \alpha-\beta\end{array}\right)$ centralizes $B$, which contradicts $A=C_{T}(B)$. So $\alpha=-1+i 2^{r-1}$ and the matrix $\left(\begin{array}{cc}\alpha & \alpha \beta \\ -\beta & \alpha-\beta\end{array}\right)$ is as claimed in (xix).

Thus there is only one nonidentity automorphism of $B$-namely inversionwhich can be induced by $C_{T}(Q)$. Since $T>A$ and $C_{T}(Q)$ is faithful on $B$, the proof of (xix) is complete.

(xx) If $A$ is of exponent $2^{r}$ for $r \geqq 3$, then every $F \cong E_{8}, F \subseteq T$ has $N_{T}(F)=\langle F, B\rangle$.

Proof. Since $[T: A]=2,[F: F \cap A]=2$ and $F \cap A=W . F=\langle f, W\rangle$ for some involution $f$ outside $A$; $f$ induces the matrix given in (xix) on $A$.

$N_{T}(F)=\langle f\rangle N_{A}(F)$ since $T=\langle f\rangle A$. We compute $N_{A}(F)$. If $\{a, b\}$ is our chosen basis for $A$, then $a^{2^{r-2}}$ and $b^{2^{r-2}}$ normalize $F$ since $f$ inverts them. But

$$
\begin{aligned}
{\left[a^{2^{r-3}}, f\right] } & =a^{-2^{r-3}}\left(a^{2^{r-3}}\right)^{f} \\
& =a^{-2^{r-3}}\left(a^{-1} w\right)^{2^{r-3}} \quad \text { for some } w \in W \\
& =a^{-2^{r-2} w^{2^{r-3}} \notin F .}
\end{aligned}
$$


Also $\left[b^{2^{r-3}}, f\right] \notin F$ and $\left[(a b)^{2^{r-3}}, f\right] \notin F$. So $N_{A}(F)=B$.

Now consider the particular four-group $F=\langle t, W\rangle$ where $C_{T}(Q)=\langle t\rangle$. By (xx), the Sylow 2-subgroups of $A_{G}(F)$ are four-groups. $F$ admits $B Q$ and so $\left|A_{G}(F)\right|$ is at least 12. Since $\left|G L_{3}(2)\right|=2^{3} \cdot 3 \cdot 7,\left|A_{G}(F)\right|$ is either $2^{2} \cdot 3$ or $2^{2} \cdot 3 \cdot 7$; the latter is impossible since $G L_{3}(2)$ has no subgroups of index 2. So $A_{G}(F)=A_{T Q}(F)$ is of order 12 with a normal four-group, namely, the stability group of the chain $F>W>1$.

$t$ is fused in $G$ to an element of $A$-hence of $W=\Omega_{1}(Z(T))$-by Lemma A. Let $T^{*}$ be a Sylow 2-subgroup of $C_{G}(t)$ (and so of $G$ ) which contains $F . N_{T^{*}}(F)=\left\langle F, B^{*}\right\rangle$ by (xx). So $N_{T^{*}}(F)$ induces a four-group on $F$, and a different four-group from that induced by $N_{T}(F)$ (since $\left.t \in W^{*}, t \notin W\right)$. But then $A_{G}(F)>A_{T Q}(F)$.

This contradiction establishes that $A$ is of exponent $4 . A=\Omega_{2}(A)=C_{T}(A)$ by (xvii), and $T / A \hookrightarrow$ Aut $(A)$.

(xxi) The subgroup of $\mathfrak{B}^{+}$induced on $A$ by $T / A$ is not contained in $C_{\mathfrak{B}}+(\sigma)$ (and hence contains $\left[\mathfrak{B}^{+}, \sigma\right]$ ).

Proof. Suppose the contrary; then $[T, Q]=A . T=[T, Q] C_{T}(Q)=A C_{T}(Q)$ splits $T$ over $A$, with $C_{T}(Q)$ elementary of order 2 or 4 .

We will show that $\Phi(T)=W$ by showing that all squares of elements of $T$ lie in $W$ (Lemma FB of $\S 1$ ). For a typical element of $T$ is $c x$ for $c \in C_{T}(Q), x \in A$; and

$$
(c x)^{2}=\operatorname{cxc} x=\operatorname{ccx}[x, c] x=c^{2} x^{2}[x, c]^{x} \in W
$$

since $c$ stabilizes the chain $A>W>1$ and so $[x, c] \in W$, and also $c^{2}=1$ and $x^{2} \in W$.

Therefore if $c$ is an involution of $C_{T}(Q),\langle c, W\rangle$ is a normal $E_{8}$ of $T$, contradicting $\mathrm{SCN}_{3}(T)$ empty.

By (xxi), $A_{T}(A) \supseteq\left[\mathfrak{B}^{+}, \sigma\right] . Q / C_{S}(T)$ acts fixed-point-freely on the four-group of $T / A$ corresponding to $\left[\mathfrak{B}^{+}, \sigma\right]$, and induces an automorphism of order 3 on the inverse image in $T$ of this four-group.

Lemma 3. Let $\sigma$ be a fixed but arbitrary 3-automorphism of $A \cong Z_{4} \times Z_{4}$. Let $R$ be an extension of $A$ by $\left[\mathfrak{B}^{+}, \sigma\right]$ such that $\sigma$ extends to an automorphism of order 3 of $R$ which is fixed-point-free on $R / A$. Then $\Phi(R)=W$, and $R$ is of one of two isomorphism types.

In detail, if a basis $\{a, b\}$ is chosen for $A$, these two isomorphism types can be described most simply as extensions of $A$ :

Split case. $R=\left\langle A, e, f, h: e\right.$ induces $\left(\begin{array}{ll}3 & 0 \\ 2 & 3\end{array}\right)$ on $A$ (i.e., $a^{e}=a^{3}$ and $\left.b^{e}=a^{2} b^{3}\right)$, $f$ induces $\left(\begin{array}{ll}3 & 2 \\ 0 & 3\end{array}\right)$ on $A, h$ induces $\left(\begin{array}{ll}1 & 2 \\ 2 & 1\end{array}\right)$ on $A ; e, f, h$ are all involutions and $e f=h$ (so that $R$ is the semidirect product of $A$ and $\langle e, f, h\rangle)\rangle$.

If $\sigma$ is taken to have the matrix $\left(\begin{array}{ll}0 & 1 \\ 3 & 3\end{array}\right)$ on $A$, then $e, f, h$ can be chosen so that $e^{\sigma}=f, f^{\sigma}=h$, and $h^{\sigma}=e$.

$G$. Higman case. $R=\left\langle A, e, f, h: e, f, h\right.$ induce $\left(\begin{array}{ll}3 & 0 \\ 2 & 3\end{array}\right),\left(\begin{array}{ll}3 & 2 \\ 0 & 3\end{array}\right),\left(\begin{array}{ll}1 & 2 \\ 2 & 1\end{array}\right)$ respectively on $A ; e^{2}=b^{2}, f^{2}=a^{2} b^{2}, h^{2}=a^{2} ; e, f, h$ commute with one another and represent the three nonidentity cosets of $W$ in the group $\langle e, f, h\rangle$, which is isomorphic to $Z_{4} \times Z_{4}$; 
if $\sigma$ is taken to have the matrix $\left(\begin{array}{cc}0 & 1 \\ 3 & 3\end{array}\right)$ on $A$, then $e, f, h$ can be chosen so that $e^{\sigma}=f$, $f^{\sigma}=h, h^{\sigma}=e$, and $\left.h=e^{-1} f^{-1}=e f a^{2}\right\rangle$.

Note. It is clear that $R$ must be of the same isomorphism types no matter which 3-matrix we take for $\sigma$, since all 3-automorphisms of $A$ are conjugate in Aut $(A)$ and so this is a matter of choice of basis in $A$.

Proof. We have seen in $\S 2$ that the matrices of $\left[\mathfrak{B}^{+}, \sigma\right]$ are the same for any choice of basis in $A$, and are $\left(\begin{array}{ll}1 & 0 \\ 0 & 1\end{array}\right),\left(\begin{array}{ll}3 & 0 \\ 2 & 3\end{array}\right),\left(\begin{array}{ll}3 & 2 \\ 0 & 3\end{array}\right)$, and $\left(\begin{array}{ll}1 & 2 \\ 2 & 1\end{array}\right)$.

Take $e \in R$ such that $e A$ induces $\left(\begin{array}{ll}3 & 0 \\ 2 & 3\end{array}\right)$ on $A$ (with respect to a basis $\{a, b\}$ of $A$ such that $\sigma$ has the matrix $\left.\left(\begin{array}{ll}0 & 1 \\ 3 & 3\end{array}\right)\right)$. Then $(e A)^{\sigma}$ induces $\left(\begin{array}{ll}3 & 0 \\ 2 & 3\end{array}\right)^{\sigma}=\left(\begin{array}{ll}0 & 1 \\ 3 & 3\end{array}\right)^{-1}\left(\begin{array}{ll}3 & 0 \\ 2 & 3\end{array}\right)\left(\begin{array}{ll}0 & 1 \\ 3 & 3\end{array}\right)$ $=\left(\begin{array}{ll}3 & 2 \\ 0 & 3\end{array}\right)$ on $A$, and $(e A)^{\sigma^{2}}$ induces $\left(\begin{array}{ll}1 & 2 \\ 2 & 1\end{array}\right)$ on $A$. So for any $f \in(e A)^{\sigma}$ and $h \in(e A)^{\sigma^{2}}, f$ induces $\left(\begin{array}{ll}3 & 2 \\ 0 & 3\end{array}\right)$ on $A, h$ induces $\left(\begin{array}{ll}1 & 2 \\ 2 & 1\end{array}\right)$ on $A$, and $e, f, h$ represent the nonidentity cosets of $A$ in $R$.

The following information will be useful (now and later):

Fixed points $\in A$

Coboundaries $\in A$

Elements of order 4 in $A$ which are inverted

$$
\begin{aligned}
& \frac{\left(\begin{array}{ll}
3 & 0 \\
2 & 3
\end{array}\right)}{W} \quad \frac{\left(\begin{array}{ll}
3 & 2 \\
0 & 3
\end{array}\right)}{W} \quad \frac{\left(\begin{array}{ll}
1 & 2 \\
2 & 1
\end{array}\right)}{W} \\
& \left\langle a^{2}\right\rangle \quad\left\langle b^{2}\right\rangle \quad\left\langle a^{2} b^{2}\right\rangle \\
& a W \quad b W \quad a b W
\end{aligned}
$$

We now observe that $R^{2}=W$ (whence $\Phi(R)=W$ by Lemma FB of $\S 1$ ). For $A^{2}=W . R^{2} \subseteq A$; and each element of $R$ which lies in a nonidentity coset of $A$ induces (by conjugation) an automorphism of $A$ whose fixed point set is just $W$; the square of such an element must lie in $W$.

$R / W \cong E_{16}$, and is fixed-point-free under $\sigma$ since $R / A$ and $A / W$ are.

Consider the coset $e A$. By the coboundary data, $(e A)^{2} \subseteq W$ is either $\left\{1, a^{2}\right\}$ or $\left\{b^{2}, a^{2} b^{2}\right\}$. We can choose the coset representative $e$ so that $e^{2}=1$ or $b^{2}$ respectively. The element $e W$ of $R / W$ belongs to some irreducible $\sigma$-submodule $L / W$ of $R / W$, with $|L|=16$ and $R / W=(L / W) \times(A / W)$. We can choose $f$ and $h$ so that $f \equiv e^{\sigma} \bmod W$ and $h \equiv f^{\sigma} \bmod W$. Then $e, f$, and $h$ represent the nonidentity cosets of $W$ in $L$ (as well as of $A$ in $R$ ). Moreover, $(e W)^{2}=e^{2}$ (since $W$ is elementary and central); therefore $f^{2}=(f W)^{2}=\left((e W)^{\sigma}\right)^{2}=\left(e^{2}\right)^{\sigma}$, and $h^{2}=\left(e^{2}\right)^{\sigma^{2}}$. So $e^{2}, f^{2}$, and $h^{2}$ are either all 1 or are $b^{2}, a^{2} b^{2}$, and $a^{2}$ respectively.

Each element of $R$ can be written in exactly one way as $e^{\alpha} f^{\beta} a^{\gamma} b^{\delta} w$, for $\alpha, \beta, \gamma, \delta$ each equal to 0 or 1 , and $w \in W$. The isomorphism type of $R$ is determined once we know how to multiply two elements of this form and get a third element of this form. We will know this once we know the commutator relations between $e, f, a$, and $b$, and the squares $(\in W)$ of $e, f, a$, and $b$. The hypotheses of Lemma 3, and our choices of $e, f$, and $h$, give us all of this information except $[e, f]$. But $L=\langle e, f, W\rangle$ satisfies the hypotheses of Lemma 2 ; so $L \cong E_{16}$ or $Z_{4} \times Z_{4}$, and $[e, f]=1$ in either case. 
So $R$ has two possible isomorphism types, corresponding in our notation to $e^{2}=1$ and $e^{2}=b^{2}$. (They differ since one contains involutions outside $W$ and the other does not.)

If $L \cong E_{16}$ and we take $f=e^{\sigma}$ and $h=f^{\sigma}$, then $\{1, e, f, h\}$ is an irreducible $\sigma$ submodule of $L$, hence a four-group, and the split case of Lemma 3 holds. ( $h^{\sigma}=e$ since $\sigma^{3}=1$.)

If $L \cong Z_{4} \times Z_{4}$ (i.e., $e^{2}=b^{2}$ ), then $\{e, f\}$ is a basis for $L$. We can in fact choose notation in $L$ so that $e^{\sigma}=f, f^{\sigma}=h$, and $h=e^{-1} f^{-1}=e f a^{2}$. For, all we know so far about the action of $\sigma$ on $L$ is its action on $W$ and on $L / W=\{W, e W, f W, h W\}$; now Aut $(L)$ has exactly four 3-elements which act as $\sigma$ does on $W$ and $L / W$, and they can all be obtained from each other by changes of basis in $L$ corresponding to similarity transformations $\Delta \in$ the stability group of the chain $L>W>1$. So if we take $\{e, f\}$ as a basis for $L$, then for each 3-matrix $M$ acting as prescribed on $W$ and $L / W$, there will be a basis $\left\{e w_{1}, f w_{2}\right\}$ of $L$ (for some $w_{1}, w_{2} \in W$ ) with respect to which $\sigma$ has the matrix $M$; $\left\{e w_{1}, f w_{2}\right\}$ is the image of $\{e, f\}$ under some $\Delta$. Since $\left\{e w_{1}, f w_{2}\right\} \equiv\{e, f\} \bmod W$, the elements $e w_{1}, f w_{2}, a, b$ satisfy the same commutatorrelations and have the same squares as $e, f, a, b$.

So far, we have specified $e, f$, and $h$ only up to congruence mod $W$; we now choose them specifically so that $\sigma$ has the matrix $\left(\begin{array}{ll}0 & 1 \\ 3 & 3\end{array}\right)$ with respect to $\{e, f\}$. (This matrix acts as prescribed on $W$ and $L / W$.) Then $e^{\sigma}=f, f^{\sigma}=h$, and $h=f^{\sigma}=e^{-1} f^{-1}=e f a^{2}$; and the $\mathrm{G}$. Higman case of Lemma 3 holds.

Write $R / A$ for the subgroup of $T / A$ corresponding to $\left[\mathfrak{B}^{+}, \sigma\right] ; R$ is then of one of the two isomorphism types named in Lemma 3.

(xxii) If $T=R$ then $T$ is of $\mathrm{G}$. Higman type.

Proof. The split $R$ has normal $E_{8}$ 's.

We will now show that $T$ must be $R$.

Assume $T>R$. Then $T=C_{T}(Q)[T, Q]=C_{T}(Q) R$ splits $T$ over $R$. For any nonidentity $c \in C_{T}(Q),\langle R, c\rangle$ is a subgroup of $T$ containing $R$ with index 2. Now $R / \Phi(R)$ is of rank 4; so by the four-generator theorem, $\Phi(\langle R, c\rangle)$ must be properly larger than $\Phi(R)=W . \Phi(T) \subseteq A$; so the only way this can happen is for [c,r] (for some $r \in R$ ) to be an element of order 4 in $A$. Then $[R, c] \cap A>W$, and $[R, c]$ is $Q$-invariant; so $[R, c]=A$.

Since $c^{2}=1,1=\left[c^{2}, r\right]=[c, r]^{c}[c, r]$, so $c$ must invert $[c, r]$. Now the automorphisms $\left(\begin{array}{ll}3 & 2 \\ 2 & 1\end{array}\right)$ and $\left(\begin{array}{ll}1 & 2 \\ 2 & 3\end{array}\right) \in C_{\mathfrak{B}}+(\sigma)$ do not invert any of the elements of order 4 in $A$. So no $c$ can occur which induces either of these automorphisms on $A$.

So if $T>R$, then $T=\langle R, z\rangle$ where $z$ inverts $A, z^{2}=1$, and $[R, z]=A$. For either of the two $R$ named in Lemma 3, the only way to have $[R, z]=A$ is for the commutators $[e, z],[f, z],[h, z]$ to be elements of order 4 in $A$.

We may assume a basis in $A$ has been chosen so that some generator $\sigma$ of $Q / C_{S}(T)$ has matrix $\left(\begin{array}{ll}0 & 1 \\ 3 & 3\end{array}\right)$ on $A$, and $e^{\sigma}=f, f^{\sigma}=h, h^{\sigma}=e$ as in Lemma 3 . Then the commutators $[e, z],[f, z]$, and $[h, z]$ are carried into one another by $Q$, and represent the three nonidentity cosets of $W$ in $A$. 
The only involutions $\in z R$ are those of $z A$. For if $k=e, f$, or $h$, and $x \in A$, then

$$
(z k x)^{2}=z k x z k x=k[k, z] x^{-1} k x \equiv k[k, z] k \bmod W .
$$

$[k, z] \in A-W$, so $k[k, z] k \in A-W$; so $(z k x)^{2} \neq 1$. Therefore, every involution of $z R$ lies in one of the four $T$-conjugate $E_{8}$ 's $\langle z, W\rangle,\left\langle z^{e}, W\right\rangle,\left\langle z^{f}, W\right\rangle$, and $\left\langle z^{h}, W\right\rangle$.

Also, $C_{T}(Z)=\left\langle z, C_{R}(z)\right\rangle$; but $z^{k}$ is $A$-conjugate to each element of $z^{k} W$ for each $k=e, f, h$, or 1 , and $z$ is conjugate to each $z^{k}$; so $\left[R: C_{R}(z)\right]=4 \cdot 4=16$, hence $\left|C_{R}(z)\right|=4$, and $C_{T}(z)=\langle z, W\rangle$. Hence the involutions of $z R$ form a single $T$ conjugacy class and the centralizer in $T$ of any one of them is $\cong E_{8}$.

Also, $N_{T}(\langle z, W\rangle)=\left\langle z, N_{R}(\langle z, W\rangle)\right\rangle$. $A$ normalizes $\langle z, W\rangle$, and induces the stability group of $\langle z, W\rangle>W\rangle 1$. If $N_{R}(\langle z, W\rangle)>A$, then $k \in N_{R}(\langle z, W\rangle)$ for $k=e, f$, or $h$; but $[z, k] \notin W$; so $N_{R}(\langle z, W\rangle)=A$. Hence each of the four $T$ conjugates $F$ of $\langle z, W\rangle$ has $N_{T}(F)=\langle F, A\rangle$, and $A$ induces the stability group of $F>W>1$.

Suppose $R$ is of G. Higman type. Then these four $E_{8}$ 's are the only $E_{8}$ 's of $T$, since $R$ contains no involutions outside $W$. So each $E_{8} F$ of $T$ has $\left|A_{G}(F)\right|$ divisible by 2 to the second power only, and $A_{T}(F)$ is the stability group of the chain $F>W>1$.

Take $F=\langle z, W\rangle$; then $T Q$ normalizes $F$, so $\left|A_{G}(F)\right|=12$ or $12 \cdot 7$ (since $\left|G L_{3}(2)\right|$ $=2^{3} \cdot 3 \cdot 7$ ). But $12 \cdot 7$ is impossible since $\mathrm{GL}_{3}(2)$ has no subgroups of index 2 . Therefore $\boldsymbol{A}_{G}(F)=\boldsymbol{A}_{T Q}(F)$ is of order 12 with a normal four-group.

By Lemma $A, z$ is fused into $R$, hence into $W$. So there is $x \in G$ with $z^{x} \in W$ and $C_{T}(z)^{x}=F^{x} \subseteq T$. By conjugating inside $T$, we may assume $F^{x}=F$. Write $T^{*}$ for $T^{x^{-1}}, W^{*}$ for $W^{x^{-1}}$; then $F \subseteq T$ and $F \subseteq T^{*}$. So $A_{G}(F)$ contains the stability group of $F>W>1$ and the stability group of $F>W^{*}>1$. These stability groups differ since $z \in W^{*}, z \notin W$. So $\boldsymbol{A}_{G}(F)>A_{T Q}(F)$, which is impossible.

Therefore $R$ splits over $A$. $T$ has several sorts of $E_{8}$ 's; we will sort the $E_{8}$ 's of $T$ (or equivalently, of $G$ ) according to the structure of their normalizers and centralizers in $T$.

We first investigate the involutions of $R$. The involutions of $A$ are the nonidentity elements of $W$. The involutions of $e A$ are the elements of $e W$ and $e a W$; these are carried by $\sigma$ into the involutions of $f A$ and $h A$, which are therefore the elements of $f W, f b W, h W$, and $h a^{-1} b^{-1} W .\{e, f, h, 1\}$ and $\left\{e a, f b, h a^{-1} b^{-1}, 1\right\}$ are both four-groups. So the involutions of $R$ all lie in the two $E_{16}$ 's,

$$
X=\langle e, f, h, W\rangle ; \quad Y=\left\langle e a, f b, h a^{-1} b^{-1}, W\right\rangle
$$

of $R . X^{z}=Y$ since $e^{z} \in e A-e W$; and $X \cap Y=W$.

Let $s$ be any involution of $X-W ; C_{T}(s) \supseteq X$. We show that $C_{T}(s)=X$. For any $r \in R, X^{2 r}=Y$; so $C_{T}(s) \subseteq R$. If $C_{T}(s)>X$, then $C_{T}(s)$ contains some element $y$ of $A-W$ (since $A / W$ is incident to $R / X$ ). But $s=k w$ for $k=e, f$, or $h$, and $w \in W$; and $[k w, y]=[k, y] \neq 1$ for $y \in A-W$. 
Our information about the involutions of $T$ now allows us to sort the $E_{8}$ 's of $T$ according to the structure of their normalizers and centralizers in $T$. We distinguish three possibilities:

Style 1. $F \nsubseteq R$. These $F$ are $\langle z, W\rangle$ and its $T$-conjugates. $N_{T}(F)=\langle F, A\rangle$; $C_{T}(F)=F$.

Style 2. $W \subseteq F \subseteq R$. Then $F \subseteq X$ or $Y ; X^{z}=Y$, so we will assume $F \subseteq X$. $N_{T}(F) \subseteq R$ (since each $z r \in z R$ sends $X$ to $Y$, and $X \cap Y=W$ ); $F \supseteq \Phi(R)$, so $N_{T}(F)=R . C_{T}(F)=X$.

Style 3. $F \subseteq R, W \nsubseteq F$. Then $F \subseteq X$ or $Y$, say $X$. $F=K \times\langle w\rangle$ for some $w \in W$ and some four-group $K$ of $X$ with $K \cap W=1$. As in Style 2, $N_{T}(F) \subseteq R . N_{T}(F) \supseteq C_{T}(F)=X$. If $N_{T}(F)>X$, then $N_{T}(F)$ contains some element $y$ of $A-W$ (since $A / W$ is incident to $R / X)$. Now $K=\left\{1, e w_{1}, f w_{2}, h w_{3}\right\}$ for $w_{1}, w_{2}, w_{3} \in W$, and

$$
\left[e w_{1}, y\right]=[e, y], \quad\left[f w_{2}, y\right]=[f, y], \quad\left[h w_{3}, y\right]=[h, y] .
$$

For fixed $y \in A-W$, these three commutators generate all of $W$. So $[F, y] \subseteq F$ is impossible for $y \in A-W$. Hence $N_{T}(F)=C_{T}(F)=X$.

(xxiii) If $F$ is of Style 1 in $T$, then $F$ cannot be of Style 2 in any Sylow 2-subgroup $T^{*}$ of $G$ with $T^{*} \supseteq F$.

Proof. If $F$ is of Style 2 in $T^{*}$, then $N_{T^{*}}(F)=R^{*}$ is a Sylow 2-subgroup of $N_{G}(F)$. Also $N_{T}(F)=\langle F, A\rangle$ is a 2-subgroup of $N_{G}(F)$, so by Sylow's theorem in $N_{G}(F)$, some conjugate of $\langle F, A\rangle$ lies in $R^{*}$. But $\langle F, A\rangle$ is a split extension of $A$ by an involution inverting $A$, and $R^{*} \cong R$ contains no subgroups of this isomorphism type.

(xxiv) If $F$ is of Style 1 in $T$, then $F$ cannot be of Style 3 in any $T^{*}$.

Proof. By (xxiii), $N_{T}(F)=\langle F, A\rangle$ is a 2-subgroup of $N_{G}(F)$ of largest possible order, so is a Sylow 2-subgroup of $N_{G}(F)$. If $F$ is of Style 3 in $T^{*}$, then $N_{T^{*}}(F) \cong E_{16}$, and by Sylow's theorem in $N_{G}(F)$, some conjugate of $N_{T^{*}}(F)$ lies in $\langle F, A\rangle$. But $\langle F, A\rangle$ contains no $E_{16}$.

(xxv) $z$ is not fused to $W$ in $G$.

Proof. Suppose the contrary. Write $F=C_{T}(z)=\langle z, W\rangle$. Since $W$ is central in $T$, there is $x \in G$ with $z^{x} \in W$ and $F^{x} \subseteq T$. By (xxiii) and (xxiv), $F^{x}$ must be of Style 1 in $T$; all the $E_{8}$ 's of Style 1 in $T$ are $T$-conjugate, so we may assume $x$ has been chosen so that $F^{x}=F$.

Now the largest power of 2 which divides $\left|A_{G}(F)\right|$ is 4 . It follows as above (by consideration of $\left.G L_{3}(2)\right)$ that $A_{G}(F)=A_{T Q}(F)$. But $T^{x}$ induces on $F$ the stability group of $F>W^{x}>1$, while $T$ induces the stability group of $F>W>1$. These stability groups differ since $z^{x} \in W, z^{x} \notin W^{x}$. Hence $A_{G}(F)>A_{T Q}(F)$, and this contradiction proves (xxv).

$z$ is fused into $R$, by Lemma A ; take $x \in G$ with $z^{x} \in R . z^{x}$ is not $G$-conjugate to $W$, by (xxv). So $C_{T}\left(z^{x}\right)=E$ say is an $E_{16}$ and is a Sylow 2-subgroup of $C_{G}\left(z^{x}\right)$. $F^{x}=C_{T}(z)^{x}$ is a 2-subgroup of $C_{G}\left(z^{x}\right)^{\prime}$, so by Sylow's theorem in $C_{G}\left(z^{x}\right)$, there is $y \in C_{G}\left(z^{x}\right)$ such that $F^{x y} \subseteq E$. But then $F^{x y}$ is of Style 2 or 3 in $T$, which contradicts (xxiii) or (xxiv). 
This contradiction completes the proof that $T=R$; so by (xxii), $T$ is of the isomorphism type claimed in Case 1.2 of Theorem 1. We showed at the beginning (before starting Case 1.1) that $N_{G}(T): C_{G}(T)=3$, 9, or 15 . If 9 , then $S / C_{S}(T)$ contains a subgroup of order 3 which centralizes $W$, so that Case 1.1 holds, which is impossible. So the proof in Case 1.2 is complete.

4. The case where $N_{G}(T)=T C_{G}(T)$ and the involutions of $W$ are all conjugate in $G$.

THEOREM 2. Suppose that $T$ is a Sylow 2-subgroup of a simple group $G ; \mathrm{SCN}_{3}(T)$ is empty but $\mathrm{SCN}_{2}(T)$ is not empty; and $T \not D_{8}$. Then (Lemma 1) Thas exactly one normal four-group, say $W$.

Suppose $N_{G}(T)=T C_{G}(T)$ and the involutions of $W$ are all conjugate in $G$.

Then one of the following holds:

Case 2.1. $T \cong Z_{2^{r}}\left\{Z_{2}(r \geqq 2)\right.$.

Case 2.2. $T$ is the semidirect product of $A \cong Z_{2^{r}} \times Z_{2^{r}}(r \geqq 3)$ by a four-group $\langle t, z\rangle$ such that $t$ interchanges the two members of some basis for $A$, and $z$ either inverts each element of $A$ or raises each element of $A$ to the power $-1+2^{r-1}$.

Case 2.3. $T$ is of order $2^{8}$, and can be described as follows:

$$
A=\langle a\rangle \times\langle b\rangle \cong Z_{4} \times Z_{4} \text {. }
$$

$R$ is the semidirect product of $A$ by a four-group $\{1, e, f, h\}$, such that $e, f, h$ have the matrices $\left(\begin{array}{ll}3 & 0 \\ 2 & 3\end{array}\right),\left(\begin{array}{ll}3 & 2 \\ 0 & 3\end{array}\right),\left(\begin{array}{ll}1 & 2 \\ 2 & 1\end{array}\right)$ respectively on $A$ (with respect to the basis $\{a, b\}$ of A).

$T$ is the semidirect product of $R$ by a four-group $\langle t, z\rangle$ such that: $z$ inverts each element of $A$, and $[z, e]=a,[z, f]=b,[z, h]=a^{-1} b^{-1} ; t$ interchanges $a$ and $b$, and $[h, t]=1,[e, t]=h,[f, t]=h$.

THEOREM 3 (the proof of which is part of the proof of Theorem 2). Suppose that $T$ is the 2-group described in Case 1.1 of Theorem 1 , and $G$ is a simple group whose Sylow 2-subgroups are isomorphic to $T$. Then $\left[N_{G}(T): C_{G}(T)\right]$ must be divisible by some odd prime (so that Case 1.1 of Theorem 1 holds in full).

Proof of Theorem 2. If $W$ is central in $T$, the three involutions of $W$ are $G$ conjugate, hence are $N_{G}(T)$-conjugate by Burnside's theorem. But this is impossible since $N_{G}(T)=T C_{G}(T)$.

So $T_{0}=C_{T}(W)$ is of index 2 in $T$.

Write $W=\left\langle z_{1}, z_{2}\right\rangle$ with $z_{1}$ central in $T$. By hypothesis there is a Sylow 2subgroup of $G$ in which $z_{2}$ is the central involution. $C_{G}\left(z_{2}\right) \supseteq T_{0}$; take $T^{*}$ to be a Sylow 2-subgroup of $C_{G}\left(z_{2}\right)$ (and so of $G$ ) such that $T^{*} \supseteq T_{0}$.

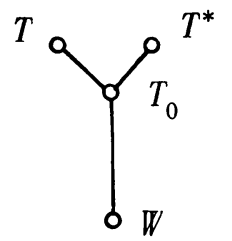


$T$ and $T^{*}$ both contain $T_{0}$ with index 2 , so both normalize $T_{0}$. So in $N_{G}\left(T_{0}\right) / T_{0}$, $T / T_{0}$ and $T^{*} / T_{0}$ generate a dihedral group $D / T_{0}$ whose order is twice an odd number.

$W=\Omega_{1}\left(Z\left(T_{0}\right)\right)$ is characteristic in $T_{0}$ so is normal in $D . T$ induces the transposition $\left(z_{1}\right)\left(z_{2}, z_{1} z_{2}\right)$ of $W-\{1\}$, while $T^{*}$ induces $\left(z_{2}\right)\left(z_{1}, z_{1} z_{2}\right)$; so $D$ induces the full symmetric group on $W-\{1\}$. So from the Sylow 3-subgroup of $D / T_{0}$, we can construct a group $S / T_{0}$ which is dihedral of order $2 \cdot 3^{n}$ for some $n ; S_{0} / T_{0}$ (say) is cyclic of order $3^{n}$, and $S_{0}=T_{0}\langle g\rangle$ where we may take $g$ to be any element of order $3^{n}$ in $S ; S=T\langle g\rangle . g$ permutes $z_{1}, z_{2}, z_{1} z_{2}$ cyclically. $g^{3}$ centralizes $T_{0}$; for if not, $g^{3}$ (being of odd order) must induce a nontrivial automorphism of $T_{0} / \Phi\left(T_{0}\right)$, but $T_{0} / \Phi\left(T_{0}\right)$ is of rank $\leqq 4$ by the four-generator theorem, and $G L_{4}(2)$ has no cyclic subgroups of order 9 .

Let $T_{0} \geqq A \geqq W$ such that $A$ is Abelian; $A \triangleleft S$; and $A$ is maximal subject to these conditions. $A$ is of rank 2 , and $\Omega_{1}(A)=W$ is cyclically permuted by $g$. Therefore $A$ is homocyclic (since otherwise $A$ could admit no nontrivial 3-automorphism, as in the proof of (i) in Case 1.1 of Theorem 1).

(i) If $A=W$, then $T_{0}=A=W$ (and hence $T \cong D_{8}$ ).

Proof. If $T_{0}>A=W$, let $Z / W=\Omega_{1}\left(Z\left(T_{0} / W\right)\right) . Z / W$ is $S$-invariant; and $T_{0}$ acts trivially on $Z / W$, so the $S$-submodules of $Z / W$ are submodules for $S /\left\langle T_{0}, g^{3}\right\rangle$ $\cong \Sigma_{3}$.

If $C_{Z / W}(g)>1$, then $C_{Z / W}(g)$ is $T$-invariant, so there is a $T$-invariant subgroup $L$ of $C_{Z / W}(g)$ with $[L: W]=2$. Then $L$ is $S$-invariant, and Abelian since $W$ is central in $L$; this contradicts the maximality of $A=W$.

So $Z / W$ is fixed-point-free under $g$. Each nonidentity element generates (under $g$ ) an irreducible module for the automorphism of order 3 induced by $g$. Every such irreducible module is a four-group, so the total number of irreducible $g$-submodules of $Z / W$ is $\frac{1}{3}(|Z / W|-1)$, which is odd. $T / T_{0}$ permutes these $g$-submodules, hence fixes one, say $K / W$. Then $K \triangleleft S ;|K|=16$, and $W$ and $K / W$ are both fixed-pointfree under $g$. By Lemma 2 (in proof of Case 1.2 of Theorem 1), $K$ is Abelian, again contrary to the maximality of $A=W$.

By (i) and the hypothesis that $T \not D_{8}$, we must have $A>W$. So $B=\Omega_{2}(A) \cong Z_{4} \times Z_{4}$. $C_{T}(B)=C_{T_{0}}(B)$ so admits $g$. Also $C_{T}(B)$ is metacyclic (Alperin [1, Corollary, p. $110]$ ); and the only metacyclic 2-group admitting an automorphism of order 3 which acts on $W$ as $g$ does, is Abelian and homocyclic. So $C_{T}(B)=A$ by the maximality of $A$.

Therefore $T / A \hookrightarrow$ Aut $(B)$; since $T_{0}=C_{T}(W), A_{T}(B) \cap \mathfrak{B}^{+}=A_{T_{0}}(B)$ (see $\S 2$ ).

Now $g$ normalizes $T_{0}$, so $A_{T_{0}}(B)\left(\subseteq B^{+}\right)$is invariant under the 3-automorphism of $B$ induced by $g$. So $A_{T_{0}}(B)$ either contains $\left[\mathfrak{B}^{+}, \sigma\right]$ or is contained in $C_{\mathfrak{B}}+(\sigma)$ (where $\sigma$ is any 3-automorphism of $B$; see $\S 2$ ). Thus either $A_{T_{0}}(B)=\left[\mathfrak{B}^{+}, \sigma\right] \times X$, or $A_{T_{0}}(B)=X$, for some subgroup $X$ of $C_{\mathfrak{B}}+(\sigma)$.

$A_{T_{0}}(B)$ is also $T$-invariant; or equivalently, $X$ is $A_{T}(B)$-invariant. Now the same subgroups $X$ of $C_{B^{+}}(\sigma)$ are $T$-invariant no matter which Sylow 2-subgroup of 
Aut $B$ we choose to contain $T$, and in fact no matter what basis we choose for $B$. These $X$ are $1,\langle Z\rangle$, and $C_{\mathfrak{B}}+(\sigma)$.

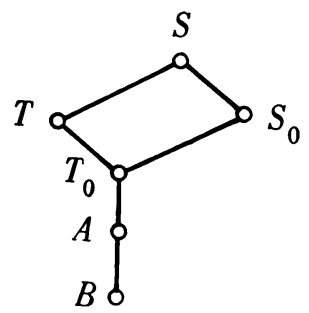

By the Frattini argument, since $\langle g\rangle$ is a Sylow 3-subgroup of $S$,

$$
S=S_{0} N_{S}(\langle g\rangle)=T_{0}\langle g\rangle N_{S}(\langle g\rangle)=T_{0} N_{S}(\langle g\rangle) .
$$

But $S=T\langle g\rangle$, so $N_{S}(\langle g\rangle)=N_{T}(\langle g\rangle)\langle g\rangle$; so,

$$
S=T_{0} N_{T}(\langle g\rangle)\langle g\rangle .
$$

But $S=T\langle g\rangle$ with $T \cap\langle g\rangle=1$, and $T_{0} N_{T}(\langle g\rangle) \subseteq T$; so

$$
T=T_{0} N_{T}(\langle g\rangle) \text {. }
$$

(ii) If $A_{T_{0}}(B) \supseteq\left[B^{+}, \sigma\right]$, let $R_{0} / A$ be the subgroup of $T_{0} / A$ corresponding to $\left[\mathfrak{B}^{+}, \sigma\right]$. Then $R_{0} \triangleleft\langle T, g\rangle=S$, and

$$
T_{0}=R_{0} C_{T_{0}}(\langle g\rangle), \quad T=R_{0} N_{T}(\langle g\rangle)
$$

where both products are semidirect.

Proof. $R_{0} \triangleleft\langle T, g\rangle$ since $\left[\mathfrak{B}^{+}, \sigma\right] \triangleleft$ Aut $\left(Z_{4} \times Z_{4}\right)$.

Since $R_{0} / A$ and $A$ are fixed-point-free under $g$, so is $R_{0}$; so $R_{0}=\left[R_{0}, g\right] \subseteq\left[T_{0}, g\right]$. But $T_{0} / R_{0}$ is centralized by $g$; therefore $\left[T_{0}, g\right]=R_{0}$. So

$$
\begin{aligned}
T_{0} & =\left[T_{0}, g\right] C_{T_{0}}(\langle g\rangle)=R_{0} C_{T_{0}}(\langle g\rangle) \\
T & =T_{0} N_{T}(\langle g\rangle)=R_{0} C_{T_{0}}(\langle g\rangle) N_{T}(\langle g\rangle)=R_{0} N_{T}(\langle g\rangle) .
\end{aligned}
$$

And $R_{0} \cap C_{T}(\langle g\rangle)=R_{0} \cap N_{T}(\langle g\rangle)=1$ since $R_{0}$ is fixed-point-free under $g$.

(iii) If $A_{T_{0}}(B) \subseteq C_{\mathfrak{B}}+(\sigma)$, then

$$
T_{0}=A C_{T_{0}}(\langle g\rangle), \quad T=A N_{T}(\langle g\rangle)
$$

where both products are semidirect.

Proof. Since $A$ is fixed-point-free under $g, A=[A, g] \subseteq\left[T_{0}, g\right]$. But $T_{0} / A$ $\hookrightarrow C_{\mathfrak{B}}+(\sigma)$, so $\left[T_{0}, g\right]=A$. So

$$
\begin{aligned}
T_{0} & =\left[T_{0}, g\right] C_{T_{0}}(\langle g\rangle)=A C_{T_{0}}(\langle g\rangle) \\
T & =T_{0} N_{T}(\langle g\rangle)=A C_{T_{0}}(\langle g\rangle) N_{T}(\langle g\rangle)=A N_{T}(\langle g\rangle) .
\end{aligned}
$$

And $A \cap C_{T_{0}}(\langle g\rangle)=A \cap N_{T}(\langle g\rangle)=1$ since $A$ is fixed-point-free under $g$. 
Case 2.1. $T_{0}=A$. We show that $T \cong Z_{2^{r}} \backslash Z_{2}$ (where $A$ is of exponent $2^{r}$ ). For $\left[T: T_{0}\right]=2 \Rightarrow T=A N_{T}\left(\langle g\rangle\right.$ ) (by (iii)) where $N_{T}(\langle g\rangle)$ is of order 2 . Now in $S / T_{0}$, $T / T_{0}$ inverts $\langle g\rangle T_{0} / T_{0}$; so in Aut $(A), T / A$ must induce a transposition of the nonidentity elements of the four-group $A / \mho^{1}(A)$. This means there is $a \in A$ with $A=\langle a\rangle \times\left\langle a^{t}\right\rangle$. Since $t^{2}=1,\left(a^{t}\right)^{t}=a$, i.e., $t$ simply exchanges $a$ and $a^{t}$.

Case 2.2. $T_{0}>A$ and $A$ of exponent $2^{r}$ for $r \geqq 3$. Each element $x$ of $T_{0}-A$ induces (by conjugation) an automorphism of $A$ which restricts to a nonidentity automorphism of $B=\Omega_{2}(A)$. $x^{2} \in A$, so this automorphism of $A$ must be of order 2 . We now show that under these conditions, certain automorphisms $\in \mathfrak{B}^{+}$cannot be induced on $B$ by $T_{0} / A$.

Choose a basis $\{a, b\}$ for $A$ such that $a^{g}=b, b^{g}=a^{-1} b^{-1}$ (such a basis exists because all 3-elements of Aut $(A)$ are conjugate in Aut $(A))$; take $\left\{a^{2^{r-2}}, b^{2^{r-2}}\right\}$ as a basis for $B$. We can then write matrices with coefficients integers mod $2^{r}$ for the automorphisms of $A$, and restriction to $B$ just means reading the matrix mod 4 .

(iv) Suppose $A$ is of exponent $2^{r}$ for $r \geqq 3$, and bases for $A$ and $B=\Omega_{2}(A)$ have been chosen as above. Then no matrix of the form ( $\left(\begin{array}{cc}2 & 2 \\ 2 & \text { odd }\end{array}\right)$ can be the restriction to $B$ of an automorphism $\theta$ of $A$ with $\theta^{2}=1$.

Proof. Let $\theta$ have matrix $\left(\begin{array}{ll}\alpha & \beta \\ \gamma & \delta\end{array}\right)$. Then

$$
\left(\begin{array}{ll}
1 & 0 \\
0 & 1
\end{array}\right) \equiv\left(\begin{array}{ll}
\alpha & \beta \\
\gamma & \delta
\end{array}\right)^{2} \equiv\left(\begin{array}{ll}
\alpha^{2}+\beta \gamma & (\alpha+\delta) \beta \\
(\alpha+\delta) \gamma & \beta \gamma+\delta^{2}
\end{array}\right) \quad \bmod 2^{r}
$$

If $\left(\begin{array}{cc}\alpha & \beta \\ \gamma & \delta\end{array}\right) \equiv\left(\begin{array}{cc}\text { odd } & 2 \\ 2 & \text { odd }\end{array}\right) \bmod 4$, then $\alpha, \delta$ are odd and $\beta, \gamma \equiv 2 \bmod 4$, so that $\beta \gamma$ $\equiv 4 \bmod 8$. But the square of any odd number is $\equiv 1 \bmod 8$; so $\alpha^{2}, \delta^{2} \equiv 1 \bmod 8$ and $\alpha^{2}+\beta \gamma \equiv 1 \bmod 8$ is impossible.

Assume in (v)-(x) that $T_{0}>A$ and $A$ is of exponent $2^{r}$ for $r \geqq 3$.

(v) $\left[T_{0}: A\right]=2$ and $T_{0}=\langle z, A\rangle$ where $z$ is an involution inverting $B . T$ is the semidirect product of $A$ by $N_{T}(\langle g\rangle)$, with $N_{T}(\langle g\rangle)=\langle z, t\rangle$ where $z$ centralizes $g$ and $t$ inverts $g$.

Proof. $\left[\mathfrak{B}^{+}, \sigma\right]$ contains the matrix $\left(\begin{array}{ll}1 & 2 \\ 2 & 1\end{array}\right)$ (with respect to any basis of $B$ ). So by (iv), $A_{T_{0}}(B) \neq\left[\mathfrak{B}^{+}, \sigma\right]$. Therefore $A_{T_{0}}(B) \subseteq C_{\mathfrak{B}^{+}}(\sigma)$. Now $C_{\mathfrak{B}^{+}}(\sigma)$ contains the matrices $\left(\begin{array}{ll}1 & 2 \\ 2 & 3\end{array}\right)$ and $\left(\begin{array}{ll}3 & 2 \\ 2 & 1\end{array}\right)$, which $T_{0} / A$ cannot induce on $B$ by (iv). So $A_{T_{0}}(B)=$ $\langle Z\rangle$ (i.e., $T_{0} / A$ inverts $B$ ). The rest of (v) follows from (iii).

(vi) The matrix of $z$ on $A$ is of the form

$$
\left(\begin{array}{rr}
-1 & 0 \\
0 & -1
\end{array}\right)+\left(\begin{array}{cc}
i & j \\
j & i+j
\end{array}\right) 2^{r-1}
$$

for some $i, j \in\{0,1\}$.

Proof. Let $z$ have matrix $\left(\begin{array}{ll}\alpha & \beta \\ \gamma & \delta\end{array}\right)$. $z$ centralizes $g$ and we have arranged that $g$ has matrix $\left(\begin{array}{rr}0 & -1 \\ -1 & -1\end{array}\right)$. So

$$
\left(\begin{array}{ll}
\alpha & \beta \\
\gamma & \delta
\end{array}\right)\left(\begin{array}{rr}
0 & 1 \\
-1 & -1
\end{array}\right) \equiv\left(\begin{array}{rr}
0 & 1 \\
-1 & -1
\end{array}\right)\left(\begin{array}{ll}
\alpha & \beta \\
\gamma & \delta
\end{array}\right) \bmod 2^{r}
$$


This is equivalent to $\gamma \equiv-\beta$ and $\delta \equiv \alpha-\beta$. So the matrix of $z$ is of the form

$$
\left(\begin{array}{cc}
\alpha & \beta \\
-\beta & \alpha-\beta
\end{array}\right)
$$

Also

$$
\left(\begin{array}{ll}
1 & 0 \\
0 & 1
\end{array}\right) \equiv\left(\begin{array}{cc}
\alpha & \beta \\
-\alpha & \alpha-\beta
\end{array}\right)^{2} \equiv\left(\begin{array}{ll}
\alpha^{2}-\beta^{2} & 2 \alpha \beta-\beta^{2} \\
\beta^{2}-2 \alpha \beta & \alpha^{2}-2 \alpha \beta
\end{array}\right) \quad \bmod 2^{r},
$$

or equivalently,

$$
\alpha^{2}-\beta^{2} \equiv 1 \bmod 2^{r}, \quad \beta^{2} \equiv 2 \alpha \beta \bmod 2^{r} .
$$

However, $\beta \equiv 0 \bmod 4$ since $z$ inverts $B$, and $\alpha$ is odd; so $\beta^{2} \equiv 2 \alpha \beta \bmod 2^{r} \Rightarrow \beta \equiv 0$ $\bmod 2^{r-1}$. Then

$$
1 \equiv \alpha^{2}-\beta^{2} \equiv \alpha^{2} \quad \bmod 2^{r},
$$

so $\alpha$ is one of the four square roots of $1 \bmod 2^{r}$, namely \pm 1 , and $\pm 1+2^{r-1}$. $\alpha \equiv-1$ mod 4 since $z$ inverts $B$; so $\alpha$ is -1 or $-1+2^{r-1}$, and (vi) is proved.

(vii) The matrix of $z$ on $A$ is

$$
\left(\begin{array}{rr}
-1 & 0 \\
0 & -1
\end{array}\right) \text { or }\left(\begin{array}{cc}
-1+2^{r-1} & 0 \\
0 & -1+2^{r-1}
\end{array}\right)
$$

Proof. $N_{T}(\langle g\rangle)=\langle t, z\rangle$ where $t$ inverts $g$ and centralizes $z$ (by (v)). Let $t$ have matrix $\left(\begin{array}{ll}\alpha & \beta \\ \gamma & \delta\end{array}\right) . g$ has matrix $\left(\begin{array}{cc}0 & -1 \\ -1 & -1\end{array}\right)$; so in order for $t$ to invert $g$, we must have

$$
\left(\begin{array}{rr}
0 & 1 \\
-1 & -1
\end{array}\right)\left(\begin{array}{ll}
\alpha & \beta \\
\gamma & \delta
\end{array}\right) \equiv\left(\begin{array}{ll}
\alpha & \beta \\
\gamma & \delta
\end{array}\right)\left(\begin{array}{rr}
-1 & -1 \\
1 & 0
\end{array}\right) \bmod 2^{r}
$$

This is equivalent to $\gamma \equiv-\alpha+\beta, \delta \equiv-\alpha \bmod 2^{r}$. So the matrix of $t$ is of the form

$$
\left(\begin{array}{cr}
\alpha & \beta \\
-\alpha+\beta & -\alpha
\end{array}\right)
$$

Also $t$ centralizes $z$, which by (vi) is equivalent to

$$
\left(\begin{array}{cc}
\alpha & \beta \\
-\alpha+\beta & -\alpha
\end{array}\right)\left(\begin{array}{cc}
i & j \\
j & i+j
\end{array}\right) \equiv\left(\begin{array}{cc}
i & j \\
j & i+j
\end{array}\right)\left(\begin{array}{cc}
\alpha & \beta \\
-\alpha+\beta & -\alpha
\end{array}\right) \bmod 2 .
$$

This gives i.e.,

$$
\left(\begin{array}{cc}
\alpha i+\beta j & \alpha j+\beta i+\beta j \\
\alpha i+\beta i+\alpha j & \beta j+\alpha i
\end{array}\right) \equiv\left(\begin{array}{cc}
\alpha i+\alpha j+\beta j & \beta i+\alpha j \\
\alpha i+\beta i+\beta j & \beta j+\alpha i+\alpha j
\end{array}\right) \bmod 2
$$

$$
\left(\begin{array}{cc}
0 & \beta j \\
\alpha j & 0
\end{array}\right) \equiv\left(\begin{array}{cc}
\alpha j & 0 \\
\beta j & \alpha j
\end{array}\right) \quad \bmod 2 .
$$

If $j=1$ this requires $\beta$ and $\alpha$ both even, in which case the matrix of $t$ is singular. So $j=0$, and (vii) follows from (vi).

(viii) If $N_{T}(\langle g\rangle)$ is cyclic, $=\langle t\rangle$ with $t^{2}=z$, then $t$ is fused into $A$. 
Proof. We consider the transfer homomorphism $\tau: G \rightarrow T / A$.

Suppose $z$ inverts $A$. Then we consider the value of $\tau$ at $t$.

$$
\tau(t)=\left(\prod\left\{y t y^{-1}: \begin{array}{l}
\text { cosets } T y \\
\text { with } T y t=T y
\end{array}\right\}\right)\left(\prod\left\{x t^{2} x^{-1}: \begin{array}{l}
\text { cosets } T x \\
\text { with } T x t \neq T x \\
\text { but } T x t^{2}=T x
\end{array}\right\}\right) A .
$$

The $x t^{2} x^{-1}$ are involutions so lie in $T_{0}$ (since $(T / A)-\left(T_{0} / A\right)$ contain no involutions $\Rightarrow T-T_{0}$ contains none). The $y t y^{-1}$ are of order 4 and so do not lie in $z A$ since $z A$ consists entirely of involutions. If no $y t y^{-1}$ lies in $A$, then $\tau(t) \equiv t A \bmod T_{0} / A$ (because the number of cosets $T y$ with $T y t=T y$ is odd), contradicting the simplicity of $G$.

Suppose $z$ raises $A$ to the power $-1+2^{r-1}$. Only one involution of $W$ is central in $T$; assume a basis $\{a, b\}$ of $A$ has been chosen so that $a^{2^{r-1}} b^{2^{r-1}} \in W$ is the central involution of $T$. (The matrix of $z$ is invariant under changes of basis in $A$. This change of basis may change the matrix $\left(\begin{array}{cc}0 & -\frac{1}{1} \\ -1 & -1\end{array}\right)$ of $g$, but we are not going to use g.) Write $\hat{a}, \hat{b}$ for $a^{2^{r-1}}, b^{2^{r-1}}$.

Consider the value of $\tau$ at $z$.

$$
\tau(z)=\prod\left\{y z y^{-1}: \quad \text { cosets } T y \text { with } T y z=T y\right\} A .
$$

The $y z y^{-1}$ are involutions so lie in $T_{0}$. If none lies in $A$, then $\tau(z)=z A$, contradicting the simplicity of $G$. So $z$ is fused into $A$, hence into $W$. The three involutions of $W$ are fused together in $G$, so $z$ is fused to $\hat{a} \hat{b}$ and there is $h \in G$ with $z^{h}=\hat{a} \hat{b}$ and $C_{T}(z)^{h} \subseteq T$.

$C_{T}(z)=\langle t, W\rangle=(\langle t\rangle \times\langle\hat{a} \hat{b}\rangle)\langle\hat{a}\rangle$ where $\hat{a}$ centralizes $\hat{a} \hat{b}$ and conjugates $t$ to $t \hat{a} \hat{b}$. $t^{2}=z$, so $\left(t^{h}\right)^{2}=z^{h}=\hat{a} \hat{b}$. Suppose $t^{h} \notin A$. There is one $T$-conjugacy-class of elements of order 4 in $T-A$ whose square is $\hat{a} \hat{b}$, and this class is represented by $z a b$; so by conjugation in $T$ we may assume $t^{h}=z a b .(\hat{a} \hat{b})^{h}$ and $(\hat{a})^{h}$ are involutions so lie in $T_{0}$; so $C_{T}(z)^{h} \subseteq T_{0}$.

$(\hat{a} \hat{b})^{h}$ must be an involution of $T_{0}$ which centralizes $z a b$ and is not $\hat{a} \hat{b}=$ $(z a b)^{2}$. We observe that $z A$ contains no such involutions (and therefore $(\hat{a} \hat{b})^{h}=$ $\hat{a}$ or $\hat{b}$ ). For if $x \in A$ and $z x$ centralizes $z a b$, then

$$
\begin{aligned}
1 & =[z a b, z x]=[z a b, x][z a b, z]^{x} \\
& =[z, x][a b, z] \equiv x^{2} a^{-2} b^{-2} \bmod W .
\end{aligned}
$$

But if $x^{2} \equiv a^{2} b^{2} \bmod W$ then $z x$ is not an involution.

So $\langle t, \hat{a} \hat{b}\rangle^{h}=\langle z a b, W\rangle$. $\hat{a}^{h}$ must be an involution of $T_{0}$ lying outside $\langle z a b, W\rangle$ (and hence $\in z A$ ), and conjugating $z a b$ to $(z a b)(\hat{a} \hat{b})^{h}$. Write $\hat{a}^{h}=z c$ for $c \in A$; then

$$
\begin{aligned}
(z a b)^{z c} & =z^{c}(a b)^{z}=c^{-1} z c(a b)^{z}=z c^{-z} c(a b)^{z} \\
& =z c^{2+2^{r-1}}(a b)^{-1+2^{r-1}} .
\end{aligned}
$$

If this equals $z a b(\hat{a} \hat{b})^{h}$, which is congruent to $z a b \bmod W$, then $c^{2} \equiv(a b)^{2} \bmod W$. But then $c \notin \mho^{1}(A)$ and so $z c$ is not an involution. 
So no such $\hat{a}^{h}$ exists. Therefore $t^{h} \in A$, and (viii) is proved.

(ix) $N_{T}(\langle g\rangle)$ is a four-group.

Proof. Suppose $N_{T}(\langle g\rangle)$ is cyclic. We survey the $T$-centralizers of the elements of order 4 in $T$.

Elements of $t A$ or $t z A$ : the centralizer in $T$ is of order 4 times the order of the centralizer in $A$. The square of any such element inverts $B$ and so the centralizer in $A$ is contained in $W$. Since $W$ is not central in $T$, this centralizer in $A$ is just $\langle\hat{a} \hat{b}\rangle$ (notation as in proof of (viii)). So the $T$-centralizer of such an element is of order 8 ; and in particular, $C_{T}(t)=\langle t, \hat{a} \hat{b}\rangle$.

Elements of $z A$ : the centralizer in $T$ is of order at most 4 times $|W|$.

Elements of $A$ : the centralizer in $T$ is $A$, of order $2^{2 r}$.

Of these three sorts of centralizers, the one of largest order is $A$.

Let $U$ be a Sylow 2-subgroup of $C_{G}(t)$. By (viii) and Sylow's theorem, $U$ is a conjugate of $A$ (for $A$, being the only Abelian maximal subgroup of $C_{T}(W)$, is characteristic in $T$ ). By Sylow's theorem in $C_{G}(t)$, we may choose $U$ to contain $C_{T}(t)=\langle t, \hat{a} \hat{b}\rangle=F$ say. Then $U$ normalizes $F$.

$N_{T}(F)=\langle F, \hat{a}\rangle$, where $a$ acts on $F$ by conjugating $t$ to $t \hat{a} \hat{b}$. For $N_{T}(F)=\left\langle t, N_{A}(F)\right\rangle$, and $N_{A}(F)$ consists of the elements $x \in A$ with $[x, t] \in\langle\hat{a} \hat{b}\rangle$. Now the map $x \rightarrow x^{-1} x^{t}$ is a homomorphism from $A$ into itself; the kernel is $C_{A}(T)=\langle\hat{a} \hat{b}\rangle$, of order 2. $N_{A}(F)$ is the inverse image under this homomorphism of $\langle\hat{a} \hat{b}\rangle$, which is of order twice $\left|C_{A}(t)\right|$, or 4 . Since $W$ normalizes $F$ by inspection, $W=N_{A}(F)$.

Let $T^{*} \supseteq R^{*} \supseteq U$ where $R^{*}$ is a Sylow 2-subgroup of $N_{G}(F)$ and $T^{*}$ is a Sylow 2-subgroup of $G$. By Sylow's theorem in $N_{G}(F)$, we may choose $R^{*}$ to contain $N_{T}(F)$. This may replace $U$ by an $N_{G}(F)$-conjugate of $U$, but the new $U$ will still contain $F$.

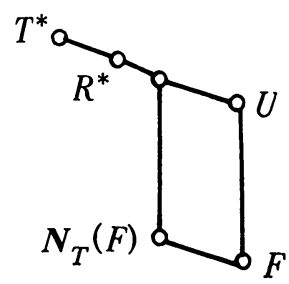

$U=A^{*}$, and $\hat{a} \in T_{0}^{*}$ since $T^{*}-T_{0}^{*}$ contains no involutions. (The characteristic subgroups $A^{*}, T_{0}^{*}$ are to $T^{*}$ as $A, T_{0}$ are to $T$.) $\hat{a}$ does not centralize $F$, so $\hat{a} \in T_{0}^{*}-U=T_{0}^{*}-A^{*}$. So $\hat{a}$ inverts $t \in \Omega_{2}\left(A^{*}\right)$. But we have just observed that $\hat{a}$ does not invert $t$. This contradiction proves (ix).

(x) $T$ is the semidirect product of $A$ by the four-group $N_{T}(\langle g\rangle)=\langle t, z\rangle$. A basis of $A$ can be chosen so that $t$ has the matrix $\left(\begin{array}{ll}0 & 1 \\ 1 & 0\end{array}\right)$ and $z$ has either the matrix $\left(\begin{array}{cc}-1 & 0 \\ 0 & -1\end{array}\right)$ or

$$
\left(\begin{array}{cc}
-1+2^{r-1} & 0 \\
0 & -1+2^{r-1}
\end{array}\right)
$$


Proof. $t$ inverts $g$, so must induce a transposition of the nonidentity elements of the four-group $A / \mho^{1}(A)$. This means there is $a \in A$ with $A=\langle a\rangle \times\left\langle a^{t}\right\rangle ;\left(a^{t}\right)^{t}=a$ since $t^{2}=1$. Take $\left\{a, a^{t}\right\}$ as basis for $A$.

Case 2.3. $T_{0}>A$ and $A \cong Z_{4} \times Z_{4}$. The remarks following (i) then hold, with $B=A$. In particular, (ii) or (iii) holds with $B=A$.

(xi) $\boldsymbol{A}_{T_{0}}(A) \supseteq\left[\mathfrak{B}^{+}, \sigma\right]$ (and (ii) holds).

Proof. Suppose $\boldsymbol{A}_{T_{0}}(A) \subseteq C_{\mathfrak{B}^{+}}(\sigma)$ (and hence (iii) holds). Now all of the automorphisms $\in C_{\mathfrak{B}^{+}}(\sigma)$ have fixed-point-set just $W$; so all squares of elements of $T_{0}-A$ lie in $W$; and also $A^{2}=W$. So $\Phi\left(T_{0}\right)=W$, by Lemma FB of $\S 1$.

Now $T_{0}>A \Rightarrow A_{T_{0}}(A)$ is either $\langle Z\rangle$ or $C_{\mathfrak{B}^{+}}(\sigma)$. In either case there is an involution $z \in C_{T_{0}}(\langle g\rangle)$ which inverts $A . z$ is central in $N_{T}(\langle g\rangle)$ since $Z$ is central in Aut $\left(Z_{4} \times Z_{4}\right) . \quad\langle z, W\rangle \cong E_{8} . \quad\left[z, N_{T}(\langle g\rangle)\right]=1$, and $\left[z, T_{0}\right] \subseteq\left[T_{0}, T_{0}\right] \subseteq W$. But $T=T_{0} N_{T}(\langle g\rangle)$; so $\langle z, W\rangle$ is a normal $E_{8}$ of $T$, contradicting $\operatorname{SCN}_{3}(T)$ empty.

Now all Sylow 3-subgroups of Aut $(A)$ are conjugate via $\left[\mathfrak{B}^{+}, \sigma\right]$; so by (xi), $\langle T, g\rangle=S$ induces every 3-automorphism of $A$. In particular, no matter which basis is taken in $A$, the matrix $\left(\begin{array}{ll}0 & 1 \\ 3 & 3\end{array}\right)$ will be induced by some 3-element of $S$. And (ii) holds no matter which Sylow 3-subgroup of $S$ is taken as $\langle\boldsymbol{g}\rangle$.

(*) So, no matter which basis is taken for $A$, we may assume $g$ has been chosen to induce $\left(\begin{array}{ll}0 & 1 \\ 3 & 3\end{array}\right)$ on $A$, and (ii) will hold for this choice of $g$.

Moreover, the automorphism $\sigma$ induced on $T_{0}$ by this $g$ will be of order exactly 3, will preserve $R_{0}$, and will act fixed-point-freely on $R_{0} / A$ (where $R_{0} / A$ is the subgroup of $T_{0} / A$ corresponding to $\left[\mathfrak{B}^{+}, \sigma\right]$, as in (ii)). So Lemma 3 (in proof of Case 1.2 of Theorem 1) applies to $R_{0}$ and $\sigma$. In particular, there are elements $e, f, h \in R_{0}$ as described in Lemma 3, with $e^{\sigma}=f, f^{\sigma}=h$, and $h^{\sigma}=e$. (Recall that Lemma 3 was a method of choosing $e, f, h \in R_{0}$ so as to satisfy the above, where $\sigma$ is any automorphism of order 3 of $R_{0}$ whose restriction to $A$ is $\left(\begin{array}{ll}0 & 1 \\ 3 & 3\end{array}\right)$ with respect to a chosen basis for $A$.)

For convenience, we will write $R$ instead of $R_{0}$.

(xii) Suppose $g \in S$ has been chosen in accordance with $\left(^{*}\right.$ ) (so that we have $R$ as in Lemma 3). Write $\sigma$ for the automorphism of $T_{0}$ induced by $g$. Suppose $Y$ is any subgroup of $T_{0}$ with $Y>R$ and $[Y: R]=2$. Then $Y=\langle y, R\rangle$ for some involution $y \in C_{T_{0}}(\langle g\rangle)$. The isomorphism type of $Y$ is completely determined by $R$, the automorphism of $A$ induced by $y$, and $[y, e]$ (for then $[y, f]=[y, e]^{\sigma},[y, h]=[y, e]^{\sigma^{2}}$ ). Furthermore, we must have $[y, e] \in A,[y, e] \notin W$.

Proof. By (ii), $T_{0}=R C_{T_{0}}(\langle g\rangle)$ where this product is semidirect and $C_{T_{0}}(\langle g\rangle)$ $\cong T_{0} / R$ is elementary. So $Y=\langle R, y\rangle$ for some involution $y \in C_{T_{0}}(\langle g\rangle)$.

$T_{0} / A$ is elementary, so $A \supseteq \Phi(Y) \supseteq \Phi(R)=W$. If $\Phi(Y)=W$ then $[Y: \Phi(Y)]=2^{5}$, which contradicts the four-generator theorem. So $\Phi(Y)>W$; since $Y$ is $\sigma$-invariant, $\Phi(Y)$ is $\sigma$-invariant and so $\Phi(Y)=A$.

$Y$ is a split extension of $R$, so its isomorphism type is completely determined by $R$ and the action of $y$ on $R$, which latter consists of the automorphism of $A$ induced by $y$ and the commutators $[y, e],[y, f]$, and (redundantly) $[y, h]$. Via $\sigma$, 
these three commutators are determined by $[y, e]$. Computation of $\Phi(Y)$ reveals that $\Phi(Y)=A$ if and only if $[y, e] \notin W$. In detail, $\Phi(Y)$ is generated by the squares of the elements of $Y$. If $x \in A$, then $[x, y] \in W$, and

$$
(x y)^{2}=x y x y=x^{2} y[y, x] y=x^{2}[y, x]^{y} \in W .
$$

If $r \in R, r \notin A$,

$$
\begin{aligned}
(r y)^{2} & =r y r y=r^{2}[y, r]^{y} \\
& \equiv[y, r] \bmod W, \quad \text { since }[y, r] \in A
\end{aligned}
$$

and $y$ stabilizes the chain $A>W>1$. So $\Phi(Y)>W$ if and only if $[y, r] \in A-W$ for some $r \in R, r \notin A$. This is equivalent to $[y, k] \in A-W$ for $k=e, f$, or $h$; but if one of these commutators lies in $A-W$, they all do since they are $\sigma$-conjugate.

(xiii) $A_{T_{0}}(A)=\mathfrak{B}^{+}$is impossible.

Proof. If $A_{T_{0}}(A)=\mathfrak{B}^{+}$, then no matter what basis is chosen for $A$, there is $y \in C_{T_{0}}(\langle g\rangle)$ inducing $\left(\begin{array}{ll}\frac{1}{2} & 2 \\ 3\end{array}\right)$ on $A \cdot y^{2}=1$, so

$$
1=\left[y^{2}, e\right]=[y, e]^{y}[y, e],
$$

and $y$ inverts $[y, e]$. But by (xii), $[y, e] \in A-W$; and $\left(\begin{array}{ll}1 & 2 \\ 2 & 3\end{array}\right)$ does not invert any element of $A-W$.

By (xiii), $A_{T_{0}}(A)$ is either $\left[\mathfrak{B}^{+}, \sigma\right]$ or $\left[\mathfrak{B}^{+}, \sigma\right] \times\langle Z\rangle$. Both of these candidates for $A_{T_{0}}(A)$ will yield $T$ with $\operatorname{SCN}_{3}(T)$ empty. In order to construct these $T$, we will choose a basis in $A$, and then take $g$ as in $\left(^{*}\right)$ so that we can use Lemma 3 to describe $R$.

$A_{T}(A)$ is contained in some Sylow 2-subgroup of Aut $(A)$. Since all the Sylow 2-subgroups of Aut $(A)$ are conjugate in Aut $(A)$, we can choose which Sylow 2subgroup of matrices will contain the matrix-group corresponding to Aut $(A)$, by a choice of basis in $A$.

(xiv) We now assume our basis $\{a, b\}$ in $A$ is one such that the matrix-group for $A_{T}(A)$ lies in $\left\langle\left(\begin{array}{ll}0 & 1 \\ 1 & 0\end{array}\right), \mathfrak{B}^{+}\right\rangle$. (There are many such bases, and we will later refine this choice.)

$\left\langle\left(\begin{array}{ll}0 & 1 \\ 1 & 0\end{array}\right), \mathfrak{B}^{+}\right\rangle$centralizes $\left(\begin{array}{ll}1 & 2 \\ 2 & 1\end{array}\right)$ and interchanges $\left(\begin{array}{ll}3 & 0 \\ 2 & 3\end{array}\right)$ and $\left(\begin{array}{ll}3 & 2 \\ 0 & 3\end{array}\right)$. So the normalization (xiv) implies that in $R / A, h A$ is centralized and $e A, f A$ interchanged by $T / A$.

(xv) Suppose $N_{T}(\langle g\rangle)=\left\langle t, N_{T_{0}}(\langle g\rangle)\right\rangle$ for $t$ an involution, and $t$ has matrix $\left(\begin{array}{ll}0 & 1 \\ 1 & 0\end{array}\right)$ on $A$. Then $R$ must split over $A$, and $[h, t] \in A$ is of the form $a^{\zeta} b^{-\zeta}$.

Proof. $[h, t] \in A$ since $h A$ is central in $T / A$. Since $t^{2}=1$,

$$
1=\left[h, t^{2}\right]=[h, t][h, t]^{t},
$$

and so $t$ must invert $[h, t]$, which is therefore of the form $a^{\xi} b^{-\zeta}$ (for some number $\zeta$ ). Also

$$
\begin{aligned}
{\left[h^{2}, t\right] } & =[h, t]^{h}[h, t]=\left(a^{\zeta} b^{-\zeta}\right)^{h}\left(a^{\zeta} b^{-\zeta}\right) \\
& =\left(a b^{2}\right)^{\zeta}\left(a^{2} b\right)^{-\zeta} a^{\zeta} b^{-\zeta}=1 .
\end{aligned}
$$

But $h^{2}=1$ if $R$ splits and $a^{2}$ if not; and $\left[a^{2}, t\right]=a^{2} b^{2} \neq 1$. 
Case 2.3.1. We now work out what $T$ has to be if $A_{T_{0}}(A)=\left[\mathfrak{B}^{+}, \sigma\right]$, i.e., $T_{0}=R$. Assume that a basis $\{a, b\}$ of $A$ has been chosen satisfying (xiv), and that $g \in S$ has then been chosen satisfying $\left({ }^{*}\right)$, so that $R$ is as described by Lemma 3 . In constructing $T$ as an extension of $R$, we will make further changes of basis in $A$; these changes of basis will not preserve the 3-matrix $\left(\begin{array}{ll}0 & 1 \\ 3 & 3\end{array}\right)$ of $g$, but will (essentially) preserve relations which hold strictly within the 2-group $R$ itself, and will also preserve (xiv).

By (ii), $T=R N_{T}(\langle g\rangle)=R\langle t\rangle$ say, for $t$ of order 2 .

(xvi) In Case 2.3.1, we may assume $t$ has the matrix $\left(\begin{array}{ll}0 & 1 \\ 1 & 0\end{array}\right)$ on $A$.

Proof. $t$ inverts $g$, where $g$ satisfies $\left({ }^{*}\right)$, i.e., the matrix of $g$ on $A$ is $\left(\begin{array}{ll}0 & 1 \\ 3 & 3\end{array}\right)$. So the matrix of $t$ is an involution $\in\left\langle\left(\begin{array}{ll}0 & 1 \\ 1 & 0\end{array}\right), \mathfrak{B}^{+}\right\rangle-\mathfrak{B}^{+}$, inverting $\left(\begin{array}{ll}0 & 1 \\ 3 & 3\end{array}\right)$. Now $\left\langle\left(\begin{array}{ll}0 & 1 \\ 1 & 0\end{array}\right), \mathfrak{B}^{+}\right\rangle$ $-\mathfrak{B}^{+}$has four involutions, namely the elements of the coset $\left(\begin{array}{ll}0 & 1 \\ 1 & 0\end{array}\right)\left\langle\left(\begin{array}{ll}3 & 0 \\ 0 & 3\end{array}\right),\left(\begin{array}{ll}1 & 2 \\ 2 & 1\end{array}\right)\right\rangle$; and of these, two-namely $\left(\begin{array}{ll}0 & 1 \\ 1 & 0\end{array}\right)$ and $\left(\begin{array}{ll}0 & 3 \\ 3 & 0\end{array}\right)$-invert $\left(\begin{array}{ll}0 & 1 \\ 3 & 3\end{array}\right)$. So the matrix of $t$ on $A$ is either $\left(\begin{array}{ll}0 & 1 \\ 1 & 0\end{array}\right)$ or $\left(\begin{array}{ll}0 & 3 \\ 3 & 0\end{array}\right)$.

If it is $\left(\begin{array}{ll}0 & 3 \\ 3 & 0\end{array}\right)$, change basis in $A$ by the similarity-matrix $\left(\begin{array}{ll}1 & 0 \\ 0 & 3\end{array}\right)$ (this is where we lose track of the 3-matrix). This change of basis does not alter (xiv), since $\left(\begin{array}{ll}1 & 0 \\ 0 & 3\end{array}\right) \in \mathfrak{B}^{+} \subseteq$ every Sylow 2-subgroup of Aut $(A)$. Moreover, $\left(\begin{array}{ll}1 & 0 \\ 0 & 3\end{array}\right)$ centralizes $\left(\begin{array}{ll}3 & 0 \\ 2 & 3\end{array}\right),\left(\begin{array}{ll}3 & 2 \\ 0 & 3\end{array}\right)$, and $\left(\begin{array}{ll}1 & 2 \\ 2 & 1\end{array}\right)$, and also centralizes every square in $R$ (namely, it centralizes $W$ ); so the relations between $e, f, h$, and $A$, and their squares, will read exactly the same after this change of basis as before. Finally, $t$ will have the matrix $\left(\begin{array}{ll}1 & 0 \\ 0 & 3\end{array}\right)^{-1}\left(\begin{array}{ll}0 & 3 \\ 3 & 0\end{array}\right)\left(\begin{array}{ll}1 & 0 \\ 0 & 3\end{array}\right)=\left(\begin{array}{ll}0 & 1 \\ 1 & 0\end{array}\right)$ with respect to the new basis.

By (xvi), (xv) applies; therefore $R$ splits over $A$, and $[h, t]=a^{5} b^{-\zeta}$ for some number $\zeta$.

$\langle h, A\rangle \triangleleft T$ and has two $E_{8}$ 's, namely $\langle h, W\rangle$ and $\langle h a b, W\rangle$. In order for $\mathrm{SCN}_{3}(T)$ to be empty, $t$ must exchange these two $E_{8}$ 's; this means $h^{t} \in h a b W$, i.e., $[h, t]=a b^{3}$ or $a^{3} b$.

(xvii) In Case 2.3.1, we may assume $[h, t]=a^{3} b$.

Proof. If $[h, t]=a b^{3}$, make the change of basis $a \rightarrow b, b \rightarrow a$ in $A$ (i.e., transform $A$ by the similarity-matrix $\left.\left(\begin{array}{ll}0 & 1 \\ 1 & 0\end{array}\right)\right)$. This does not affect (xiv), and does not change the matrix of $t$ on $A$. $\left(\begin{array}{ll}0 & 1 \\ 1 & 0\end{array}\right)$ centralizes $\left(\begin{array}{ll}1 & 2 \\ 2 & 1\end{array}\right)$ and exchanges $\left(\begin{array}{ll}3 & 0 \\ 2 & 3\end{array}\right)$ and $\left(\begin{array}{ll}3 & 2 \\ 0 & 3\end{array}\right)$; so in $R, h$ will have the same matrix as before, and $e$ will have the matrix $f$ had and $f$ will have the matrix $e$ had, with respect to the new basis. $e^{2}, f^{2}$, and $[e, f]$ will read the same after the change of basis as before, since they are equal to 1 . So if after making this change of basis, we reverse the roles of the letters $e$ and $f$, we will get $R$ again exactly as in the statement of Lemma 3 . Finally, $[h, t]$ will appear as $a^{3} b$ with respect to the new basis.

To construct $T$ as a split extension of $R$, it remains only to determine [e,t] and (redundantly) $[f, t]$. We now work out what $[e, t]$ and $[f, t]$ are, without making any more changes of basis.

Since $T / A$ interchanges $e A$ and $f A,[e, t]$ and $[f, t]$ lie in $h A$. Write $[e, t]=h x$, $[f, t]=h y$ for $x, y \in A$. Now $e f=h$, so $[e, t]$ determines $[f, t]$ as follows:

$$
a^{3} b=[h, t]=[e f, t]=[e, t]^{f}[f, t]=(h x)^{f}(h y) .
$$


Since $1, e, f, h$ form a four-group, we have

$$
a^{3} b=f h x f h y=\text { exey }=x^{e} y .
$$

We now compute $[e, t]$. Since $e^{2}=1$,

$$
1=\left[e^{2}, t\right]=[e, t]^{e}[e, t],
$$

so $e$ inverts $[e, t]$. Similarly $t^{2}=1 \Rightarrow t$ inverts $[e, t]$. The inverse of a typical element $h a^{\xi} b^{\eta}$ of $h A$ is $h a^{-\xi+2 \eta} b^{-\eta+2 \xi}$. If we write $[e, t]=h x=h a^{\alpha} b^{\beta}$, then inversion by $e$ gives

$$
\begin{aligned}
\left(h a^{\alpha} b^{\beta}\right)^{e} & =h a^{-\alpha+2 \beta} b^{-\beta+2 \alpha} \\
\| & \\
h\left(a^{3}\right)^{\alpha}\left(a^{2} b^{3}\right)^{\beta} & =h a^{-\alpha+2 \beta} b^{-\beta},
\end{aligned}
$$

or equivalently, $-\beta+2 \alpha=-\beta$, so that $\alpha$ is even. Inversion by $t$ gives

$$
\begin{aligned}
\left(h a^{\alpha} b^{\beta}\right)^{t} & =h a^{-\alpha+2 \beta} b^{-\beta+2 \alpha} \\
\| & \\
h a^{3} b a^{\beta} b^{\alpha} & =h a^{\beta+3} b^{\alpha+1},
\end{aligned}
$$

or equivalently, $\beta+3=-\alpha+2 \beta, \alpha+1=-\beta+2 \alpha$, or equivalently, $\beta=3+\alpha$, so that $\beta$ is determined by $\alpha$.

So we seem to have two groups $T$ : one with $\alpha=0$ (i.e., $[e, t]=h b^{3}$ ) and one with $\alpha=2$ (i.e., $[e, t]=h a^{2} b$ ).

But these two $T$ are isomorphic. For if we take $\hat{a}=a, \hat{b}=b ; \hat{e}=e a^{2}, \hat{f}=f b^{2}$, $\hat{h}=h a^{2} b^{2} ; \hat{t}=t a^{2} b^{2}$, then the matrices of $\hat{e}, \hat{f}, \hat{h}, \hat{t}$ on $A$ (with respect to $\{\hat{a}, \hat{b}\}=\{a, b\}$ ) are the same as those of $e, f, h, t ; \hat{e} \hat{f}=\hat{h}$ and $\hat{e}, \hat{f}, \hat{h}$ are involutions; $[\hat{h}, \hat{t}]=[h, t]$ since $a^{2} b^{2}$ is central; and

$$
[\hat{e}, \hat{t}]=\left[e a^{2}, t a^{2} b^{2}\right]=\left[e a^{2}, t\right]=[e, t] a^{2} b^{2} .
$$

So in Case 2.3.1,

$$
\begin{aligned}
& T=\left\langle\text { split } R, t: t^{2}=1, t \text { has matrix }\left(\begin{array}{ll}
0 & 1 \\
1 & 0
\end{array}\right)\right. \text { on A; } \\
& \left.\qquad[h, t]=a^{3} b, \quad[e, t]=h b^{3}, \quad[f, t]=h a\right\rangle .
\end{aligned}
$$

(Note that these relations do define a group, since the relations given between $t$ and $R$ define an automorphism of order 2 of the group $R$.)

This $T$ has $\mathrm{SCN}_{3}(T)$ empty. For if not, $T$ has a normal $E_{8}, F$ say, containing $W$ (by the Lemma below). $F \subseteq C_{T}(W)=T_{0}$, and projects onto exactly one nonidentity coset of $A$ in $T_{0}$. This coset must be $h A$ since $(e A)^{t}=f A$. But we have explicitly arranged that no $E_{8} \subseteq\langle h, A\rangle$ is normal in $T$.

LEMMA. If $W$ is a normal four-group of any 2-group $T$, and $T$ has a normal $E_{8}(F$ say), then $T$ has a normal $E_{8}$ containing $W$.

Proof. $C_{F}(W) \triangleleft T$, contains $F \cap W$, and is of order $\geqq 4$ because $\mid$ Aut $(W) \mid=6$. If $F$ $W$, then $|F \cap W| \leqq 2$, so $\left|C_{F}(W) W\right| \geqq(4 \cdot 4) / 2=8$; so $C_{F}(W) W$ is a normal 
elementary subgroup of $T$ of order $\geqq 8$, containing $W$. So there is $K$ with $C_{F}(W) W$ $\geqq K>W$ such that $K \triangleleft T$ and $K \cong E_{8}$.

Now suppose $A_{T_{0}}(A)=\left[\mathfrak{B}^{+}, \sigma\right] \times\langle Z\rangle$. Assume that a basis $\{a, b\}$ of $A$ has been chosen satisfying (xiv), and that $g \in S$ has then been chosen satisfying (*), so that $R$ is as described by Lemma 3.

(xviii) If $A_{T_{0}}(A)=\left[\mathfrak{B}^{+}, \sigma\right] \times\langle Z\rangle$, then $T_{0}=\langle z\rangle R$ is a split extension of $R$ by an involution which inverts $A$ and centralizes $g$. Moreover, we may assume (by choice of basis in $A$ ) that $[z, e]=a$; and $[z, f]=a^{\sigma}=b ;[z, h]=b^{\sigma}=a^{-1} b^{-1}$.

Proof. The first statement follows from (ii).

Taking $Y=T_{0}$ in (xii), we see that $[z, e] \in A-W$ and $[z, e]$ (together with $R$ ) completely determines $T_{0}$. Since $e^{2} \in W$,

$$
1=\left[z, e^{2}\right]=[z, e][z, e]^{e},
$$

so $e$ must invert $[z, e]$. Therefore $[z, e] \in a W$.

Let $\Delta$ be one of the matrices $\left(\begin{array}{ll}1 & 0 \\ 0 & 1\end{array}\right),\left(\begin{array}{ll}3 & 0 \\ 0 & 3\end{array}\right),\left(\begin{array}{ll}1 & 2 \\ 2 & 3\end{array}\right),\left(\begin{array}{ll}3 & 2 \\ 2 & 1\end{array}\right)$. Then $\Delta$ centralizes $\mathfrak{B}^{+}$ and $\left(\begin{array}{ll}0 & 1 \\ 3 & 3\end{array}\right)$; so if we change basis in $A$ by $\Delta$, the matrices of $e, f, h$, and $\sigma$ will read exactly the same after the change of basis as before. In particular, $\left(^{*}\right)$ still holds with respect to the new basis, so (xii) still applies.

$[z, e]$ will be replaced by $[z, e]^{\Delta}$. Now these matrices form a group which acts transitively on the elements of $a W$; so there is a unique $\Delta$ such that $[z, e]^{\Delta}=a$. Having made the change of basis $\{a, b\} \rightarrow\left\{a^{\Delta}, b^{\Delta}\right\}$ for this $\Delta$, we will have

$$
\begin{aligned}
& {[z, e]=a,} \\
& {[z, f]=\left[z^{\sigma}, e^{\sigma}\right]=a^{\sigma}=b}
\end{aligned}
$$

(since the matrix of $\sigma$ is unchanged)

$$
[z, h]=b^{\sigma}=a^{-1} b^{-1} .
$$

Now $T=R N_{T}(\langle g\rangle)$, where $N_{T}(\langle g\rangle)$ is either a four-group or cyclic of order 4 .

Case 2.3.2. We now work out what $T$ has to be if $N_{T}(\langle g\rangle)$ is a four-group, say $\langle t, z\rangle$ where $t$ is an involution inverting $g$.

(xix) In Case 2.3.2, we may assume $t$ has the matrix $\left(\begin{array}{ll}0 & 1 \\ 1 & 0\end{array}\right)$ on $A$.

Proof. As in (xvi), the matrix of $t$ is either $\left(\begin{array}{ll}0 & 1 \\ 1 & 0\end{array}\right)$ or $\left(\begin{array}{ll}0 & 3 \\ 3 & 0\end{array}\right)$; if it is $\left(\begin{array}{ll}0 & 3 \\ 3 & 0\end{array}\right)$, replace $t$ by $t z$.

By (xix), (xv) applies; therefore $R$ splits over $A$, and $[h, t]=a^{\zeta} b^{-\zeta}$ for some number $\zeta$. [e,t] and $[f, t]$ lie in $h A$ since $T / A$ interchanges $e A$ and $f A$; write $[e, t]=h x$, $[f, t]=h y$; for $x, y \in A$. (Once we determine $[e, t],[f, t]$, and $[h, t], T$ is completely determined.)

As in Case 2.3.1, $[e, t]$ determines $[f, t]$ once $[h, t]$ has been chosen; for

$$
[h, t]=[e f, t]=[e, t]^{f}[f, t]=x^{e} y \quad(\text { as in Case 2.3.1). }
$$

Writing $x=a^{\alpha} b^{\beta}, y=a^{\gamma} b^{\delta}$, this gives

$$
a^{\zeta} b^{-\zeta}=\left(a^{\alpha} b^{\beta}\right)^{e}\left(a^{\gamma} b^{\delta}\right)=a^{3 \alpha}\left(a^{2} b^{3}\right)^{\beta} a^{\gamma} b^{\delta}=a^{-\alpha+2 \beta+\gamma} b^{-\beta+\delta},
$$

or equivalently, $\zeta=-\alpha+2 \beta+\gamma=\beta-\delta$. 
Also, $e^{2}=1$ and $t^{2}=1 \Rightarrow[e, t]$ is inverted by $e$ and $t$. Inversion by $e$ gives $\alpha$ even, as in Case 2.3.1. Inversion by $t$ gives

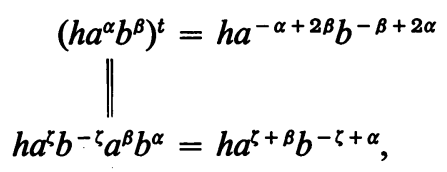

or equivalently, $-\alpha+2 \beta=\zeta+\beta,-\beta+2 \alpha=-\zeta+\alpha$, or equivalently, $-\alpha+\beta=\zeta$. So we have

$$
\zeta=-\alpha+2 \beta+\gamma=\beta-\delta=-\alpha+\beta .
$$

$(1)=(3)$ gives

$$
0=\beta+\gamma
$$

$(2)=(3)$ gives

$$
0=-\alpha+\delta
$$

Also, conjugation of $T_{0}$ by $t$ must preserve the relations $a=[z, e], b=[z, f]$. This gives

$$
b=a^{t}=\left[z^{t}, e^{t}\right]=[z, f x]=[z, x][z, f]^{x}=[z, x] b
$$

so $[z, x]=1$, so $x \in W$. Therefore $\alpha$ and $\beta$ are even; so by (\% $\%), \gamma$ and $\delta$ are also even; by $(\%), \zeta$ is even and

$$
(\% \% \%) \quad \zeta=-\alpha+\beta .
$$

So once $\zeta=0$ or 2 has been chosen, $\alpha=0$ or 2 determines $\beta, \gamma$, and $\delta$ via $(\% \%)$ and $(\% \% \%)$.

So we get four $T$, all of which are split extensions of $T_{0}$ as in (xviii) (for split $R$ ) by an involution $t$ centralizing $z$ and having matrix $\left(\begin{array}{ll}0 & 1 \\ 1 & 0\end{array}\right)$ on $A$, and defined by the following four sets of relations:

(1) $\zeta=2$ and $\alpha=2$ :

$$
[h, t]=a^{2} b^{2}, \quad[e, t]=h a^{2}, \quad[f, t]=h b^{2} .
$$

(2) $\zeta=2$ and $\alpha=0$ :

$$
[h, t]=a^{2} b^{2}, \quad[e, t]=h b^{2}, \quad[f, t]=h a^{2} .
$$

(3) $\zeta=0$ and $\alpha=2$ :

$$
[h, t]=1, \quad[e, t]=h a^{2} b^{2}, \quad[f, t]=h a^{2} b^{2} .
$$

(4) $\zeta=0$ and $\alpha=0$ :

$$
[h, t]=1, \quad[e, t]=h, \quad[f, t]=h .
$$


These four sets of relations do define groups. Indeed, we will show that the four sets are equivalent (by choosing new generators), so that they all define groups if and only if one does, and the groups are isomorphic. And (4) is easily seen to define a group since the relations given between $t$ and $T_{0}$ define an automorphism of order 2 of $T_{0}$.

The $T$ of (4) has $\mathrm{SCN}_{3}(T)$ empty. For if not, $T$ has a normal $E_{8}, F$ say, containing $W . F \subseteq C_{T}(W)=T_{0}$ and projects onto precisely one nonidentity coset of $A$ in $T_{0}$. This coset is not $e A, f A$, or $h A$ because the action of $z$ prevents any such $E_{8}$ from being normal in $T$. The only remaining coset of $A$ in $T_{0}$ which contains involutions is $z A$. For $x \in A$,

$$
(z x)^{h}=z a^{-1} b^{-1} x^{h} \equiv z a^{-1} b^{-1} x \bmod W,
$$

so that $\langle z x, W\rangle^{n} \neq\langle z x, W\rangle$.

We now show that (1), (2), (3), (4) are equivalent. Consider first

$$
\begin{array}{ll}
\hat{t}=t a b^{-1} ; & \hat{e}=e a^{2}, \hat{f}=f b^{2}, \hat{h}=h a^{2} b^{2} ; \\
\hat{z}=z a^{2} . & (\hat{a}=a, \hat{b}=b .)
\end{array}
$$

Then the restriction of ${ }^{\wedge}$ to $T_{0}$ preserves the relations defining $T_{0}$. (The relations defining $T_{0}$ are of the form

$$
\begin{array}{rlrl}
x^{\alpha} & =1 & & \left(x \in T_{0}\right) ; \\
{[x, y]} & =z & & \left(x, y \in T_{0}, z \in A\right) ; \\
e f & =h . &
\end{array}
$$

The first two sorts of relations are automatically preserved by $\hat{\uparrow}$, since the commutators by ${ }^{\wedge}$ are central involutions of $T_{0}$ and ${ }^{\wedge}$ is the identity on $A$; and $e f=h$ is also preserved by ${ }^{\wedge}$.)

$$
\begin{aligned}
{[\hat{z}, \hat{t}] } & =\left[z a^{2}, t a b^{-1}\right]=\left[z, t a b^{-1}\right]^{a^{2}}\left[a^{2}, t a b^{-1}\right] \\
& =\left[z, a b^{-1}\right][z, t]^{a b-1}\left[a^{2}, t\right]=a^{2} b^{2}[z, t] a^{2} b^{2} \\
& =[z, t] . \\
{[\hat{h}, \hat{t}] } & =\left[h a^{2} b^{2}, t a b^{-1}\right]=\left[h, t a b^{-1}\right]=\left[h, a b^{-1}\right][h, t]^{a b^{-1}} \\
& =a^{2} b^{2}[h, t] . \\
{[\hat{e}, \hat{t}] } & =\left[e a^{2}, t a b^{-1}\right]=\left[e, t a b^{-1}\right]^{a^{2}}\left[a^{2}, t a b^{-1}\right] \\
& =\left[e, a b^{-1}\right][e, t]^{a b^{-1}}\left[a^{2}, t\right] \\
& =e^{-1}\left(a^{-1} b\right) e a b^{-1}\left(a b^{-1}\right)^{-1}[e, t]\left(a b^{-1}\right)\left[a^{2}, t\right] \\
& =\left(a^{-1} b\right)^{e}[e, t] a b^{-1} a^{2} b^{2} \\
& =[e, t]\left(a^{-1} b\right)^{f} a b^{-1} a^{2} b^{2} \quad \text { since } \quad[e, t] \in h A \quad \text { and } e h=f \\
& =[e, t]\left(a b^{2} b^{-1}\right) a b^{-1} a^{2} b^{2} \\
& =[e, t] b^{2} . \\
{[\hat{f}, \hat{t}] } & =[f, t] a^{2} .
\end{aligned}
$$


So the switch to hats gives (1) $\leftrightarrow(3),(2) \leftrightarrow(4)$.

Consider next

$$
\begin{aligned}
& \hat{t}=t a^{2} b^{2} ; \quad \hat{e}=e a^{2}, \hat{f}=f b^{2}, \hat{h}=h a^{2} b^{2} ; \\
& \hat{z}=z . \quad(\hat{a}=a, \hat{b}=b .)
\end{aligned}
$$

The restriction of ${ }^{\wedge}$ to $T_{0}$ preserves the relations defining $T_{0}$ (as before).

$$
\begin{aligned}
& {[\hat{z}, \hat{t}]=[z, t] .} \\
& {[\hat{h}, \hat{t}]=[h, t] .} \\
& {[\hat{e}, \hat{t}]=\left[e a^{2}, t a^{2} b^{2}\right]=[e, t] a^{2} b^{2} .} \\
& {[\hat{f}, \hat{t}]=[f, t] a^{2} b^{2} .}
\end{aligned}
$$

So the switch to hats gives (1) $\leftrightarrow(2)$, (3) $\leftrightarrow(4)$.

Therefore all four sets of relations are equivalent.

Case 2.3.3. We now work out what $T$ has to be if $N_{T}(\langle g\rangle)$ is cyclic of order 4, say $\langle t\rangle$ where $t^{2}=z$.

(xx) In Case 2.3.3, we may assume $t$ has the matrix $\left(\begin{array}{ll}2 & 3 \\ 1 & 2\end{array}\right)$ on $A$.

Proof. $t$ inverts $g$, where $g$ satisfies $\left({ }^{*}\right)$, i.e., the matrix of $g$ on $A$ is ${ }^{*}\left(\begin{array}{ll}0 \\ 3\end{array}\right]$ s $;$; and $t^{2}=z$; so the matrix of $t$ is an element of $\left\langle\left(\begin{array}{ll}0 & 1 \\ 1 & 0\end{array}\right), \mathfrak{B}^{+}\right\rangle-\mathfrak{B}^{+}$whose square is $\left(\begin{array}{ll}3 & 0 \\ 0 & 3\end{array}\right)$ and which inverts $\left(\begin{array}{ll}0 & 1 \\ 3 & 3\end{array}\right)$. Now $\left\langle\left(\begin{array}{ll}0 & 1 \\ 1 & 0\end{array}\right), \mathfrak{B}^{+}\right\rangle-\mathfrak{B}^{+}$has four elements whose square is $\left(\begin{array}{ll}3 & 0 \\ 0 & 3\end{array}\right)$, namely the elements of the coset $\left(\begin{array}{ll}2 & 3 \\ 1 & 2\end{array}\right)\left\langle\left(\begin{array}{ll}3 & 0 \\ 0 & 3\end{array}\right),\left(\begin{array}{ll}1 & 2 \\ 2 & 1\end{array}\right)\right\rangle$; and two of thesenamely $\left(\begin{array}{ll}2 & 3 \\ 1 & 2\end{array}\right)$ and $\left(\begin{array}{ll}3 & 0 \\ 0 & 3\end{array}\right)\left(\begin{array}{ll}2 & 3 \\ 1 & 2\end{array}\right)$-invert $\left(\begin{array}{ll}0 & 1 \\ 3 & 3\end{array}\right)$. If the matrix of $t$ is $\left(\begin{array}{ll}3 & 0 \\ 0 & 3\end{array}\right)\left(\begin{array}{ll}2 & 3 \\ 1 & 2\end{array}\right)$, replace $t$ by $t z$.

(xxi) In Case 2.3.3 (assuming $t$ has the matrix $\left(\begin{array}{ll}2 & 3 \\ 1 & 2\end{array}\right)$ on $A$ ), $[h, t]$ is either $a b^{2}$ or $a^{3}$.

Proof. $t^{2}=z$, and $[h, z]=a b$ by (xviii); so

$$
a b=[h, z]=\left[h, t^{2}\right]=[h, t][h, t]^{t} .
$$

Also, $[h, t] \in A$ since $h A$ is central in $T / A$. Writing $[h, t]=a^{\xi} b^{\eta}$, we have

$$
a b=\left(a^{\xi} b^{\eta}\right)\left(a^{\xi} b^{\eta}\right)^{t}=a^{\xi} b^{\eta}\left(a^{2} b^{3}\right)^{\xi}\left(a b^{2}\right)^{\eta}=a^{3 \xi+\eta} b^{3 \eta+3 \xi},
$$

so that $\xi, \eta$ must satisfy $1=3 \xi+\eta, 1=3 \xi+3 \eta$. There are two solutions, namely $\eta=2$ and $\xi=1 ; \eta=0$ and $\xi=3$.

(xxii) In Case 2.3.3, $R$ is of $\mathrm{G}$. Higman type.

Proof. If $R$ splits, then $h^{2}=1$ and so $h$ inverts [h,t]. But this is impossible by (xxi), as $h$ does not invert $a b^{2}$ or $a^{3}$.

So $T$ is completely determined once we determine $[e, t],[f, t]$, and $[h, t] .[e, t]$ and $[f, t]$ lie in $h A$ since $T / A$ interchanges $e A$ and $f A$. Write $[e, t]=h x=h a^{\alpha} b^{\beta}$, $[f, t]=h y=h a^{\gamma} b^{\delta}($ for $x, y \in A)$. 
As in the first two cases, $[e, t]$ determines $[f, t]$ once $[h, t]$ has been chosen; for $h=e f a^{2}$, and

$$
\begin{aligned}
{[h, t] } & =\left[e f a^{2}, t\right]=[e f, t]^{a}\left[a^{2}, t\right]=[e, t]^{f}[f, t] a^{2} b^{2} \\
& =(h x)^{f}(h y) a^{2} b^{2}=f^{-1}(h x) f(h y) a^{2} b^{2}=h^{2}\left(h^{-1} f^{-1}\right) x(f h) y a^{2} b^{2} \\
& =h^{2}\left(x^{e} y\right) a^{2} b^{2}, \quad \text { since } f h \equiv e \bmod \text { center of } R \\
& =b^{2} x^{e} y \\
& =b^{2}\left(a^{\alpha} b^{\beta}\right)^{e}\left(a^{\gamma} b^{\delta}\right)=b^{2} a^{-\alpha+2 \beta+\gamma} b^{-\beta+\delta} .
\end{aligned}
$$

In summary, the relation between $[e, t],[f, t]$, and $[h, t]$ is

$$
[h, t]=b^{2} a^{-\alpha+2 \beta+\gamma} b^{-\beta+\delta} \text {. }
$$

Also, $t^{2}=z$ and $[e, z]=a^{-1}$; so

$$
\begin{aligned}
a^{-1} & =[e, z]=\left[e, t^{2}\right]=[e, t][e, t]^{t}=(h x)(h x)^{t} \\
& =(h x) h[h, t] x^{t} \\
& =h^{2} x^{h} x^{t}[h, t] \\
& =a^{2}\left(a b^{2}\right)^{\alpha}\left(a^{2} b\right)^{\beta}\left(a^{2} b^{3}\right)^{\alpha}\left(a b^{2}\right)^{\beta}[h, t]
\end{aligned}
$$

so that

$(\% \%)$

$$
a^{-1}=a^{2} a^{-\alpha-\beta} b^{\alpha-\beta}[h, t]
$$

Also, $f^{2}=a^{2} b^{2}$; so

$$
\begin{aligned}
1 & =\left[a^{2} b^{2}, t\right]=\left[f^{2}, t\right]=[f, t]^{f}[f, t]=(h y)^{f}(h y) \\
& =f^{-1}(h y) f(h y) \\
& =h^{2} y^{e} y, \quad \text { since } f h \equiv e \bmod \text { center of } R \\
& =a^{2}\left(a^{\gamma} b^{\delta}\right)^{e}\left(a^{\gamma} b^{\delta}\right)=a^{2} a^{3 \gamma}\left(a^{2} b^{3}\right)^{\delta} a^{\gamma} b^{\delta} \\
& =a^{2} a^{2 \delta} .
\end{aligned}
$$

Therefore $\delta$ must be odd.

We now apply $(\%)$ and $(\% \%)$. with our two values for $[h, t]$. First suppose $[h, t]=a b^{2} ;$ then $(\%)$ yields

$$
1=-\alpha+2 \beta+\gamma, \quad 0=-\beta+\delta, \text { i.e., } \beta=\delta .
$$

Thus $\beta$ is odd, so $2 \beta=2$ and the first equation becomes $\gamma=\alpha-1$. (\%\%) yields $0=-\alpha-\beta, 2=\alpha-\beta$, or equivalently (since $\beta$ is odd), $\beta=-\alpha$. So $\alpha$ is odd; and $\alpha$ determines $\beta, \gamma$, and $\delta$, hence completely determines $T$.

Next, suppose $[h, t]=a^{3}$; then $(\%)$ yields

$$
-1=-\alpha+2 \beta+\gamma, \quad 2=-\beta+\delta \text {, i.e., } \beta=2+\delta .
$$

Thus $\beta$ is odd, so $2 \beta=2$ and the first equation becomes $\gamma=\alpha+1$. (\%\%) yields $2=-\alpha-\beta, 0=\alpha-\beta$, or equivalently (since $\beta$ is odd), $\beta=\alpha$. So $\alpha$ is odd; and $\alpha$ determines $\beta, \gamma$, and $\delta$, hence completely determines $T$. 
So we get four $T$, all of which are extensions of $T_{0}$ as in (xviii) (for nonsplitting $R$ ) by an element $t$ with $t^{2}=z$, where $t$ has matrix $\left(\begin{array}{ll}2 & 3 \\ 1 & 2\end{array}\right)$ on $A$. The $T$ are defined by the following four sets of relations:

(1) $[h, t]=a b^{2}$ and $\alpha=1$ :

$$
[h, t]=a b^{2}, \quad[e, t]=h a b^{3}, \quad[f, t]=h b^{3} .
$$

(2) $[h, t]=a b^{2}$ and $\alpha=3$ :

$$
[h, t]=a b^{2}, \quad[e, t]=h a^{3} b, \quad[f, t]=h a^{2} b .
$$

(3) $[h, t]=a^{3}$ and $\alpha=1$ :

$$
[h, t]=a^{3}, \quad[e, t]=h a b, \quad[f, t]=h a^{2} b^{3} .
$$

(4) $[h, t]=a^{3}$ and $\alpha=3$ :

$$
[h, t]=a^{3}, \quad[e, t]=h a^{3} b^{3}, \quad[f, t]=h b .
$$

These four sets of relations do define groups. Indeed, we will show that the four sets are equivalent (by choosing new generators), so that they all define groups if and only if one does, and the groups are isomorphic. And (4) defines a group because the relations given between $t$ and $T_{0}$ define an automorphism of $T_{0}$ whose square is the inner automorphism induced by $z$.

The $T$ of (4) has $\operatorname{SCN}_{3}(T)$ empty. For if not, $T$ has a normal $E_{8}, F$ say, containing $W$. $F \subseteq C_{T}(W)=T_{0}$ and projects onto precisely one nonidentity coset of $A$ in $T_{0}$. The only such coset containing involutions is $z A$; and for $x \in A,\langle z x, W\rangle^{h} \neq\langle z x, W\rangle$ as in Case 2.3.2.

We now show that (1), (2), (3), (4) are equivalent. Consider first $\hat{t}=t a b^{-1}$; $\hat{e}=e a^{2}, \hat{f}=f b^{2}, \hat{h}=h a^{2} b^{2} .\left(\hat{a}=a, \hat{b}=b\right.$.) Then $\hat{z}=(t)^{2}=\left(t a b^{-1}\right)^{2}=z a^{2}$. Then (as in Case 2.3.2) the restriction of ${ }^{\wedge}$ to $T_{0}$ preserves the relations defining $T_{0}$.

Computing as in Case 2.3.2, we get

$$
\begin{aligned}
& {[\hat{h}, \hat{t}]=[h, t] a^{2} b^{2}} \\
& {[\hat{e}, \hat{t}]=[e, t] b^{2} .} \\
& {[\hat{f}, \hat{t}]=[f, t] a^{2} .}
\end{aligned}
$$

So the switch to hats gives (1) $\leftrightarrow(3),(2) \leftrightarrow(4)$.

Consider next $\hat{t}=t a^{2} b^{2} ; \hat{e}=e a^{2}, \hat{f}=f b^{2}, \hat{h}=h a^{2} b^{2} .(\hat{a}=a, \hat{b}=b$. $)$ Then $\hat{z}=(\hat{t})^{2}$ $=\left(t a^{2} b^{2}\right)^{2}=z$. The restriction of ${ }^{\wedge}$ to $T_{0}$ preserves the relations defining $T_{0}$. As in Case 2.3.2,

$$
\begin{aligned}
& {[\hat{h}, \hat{t}]=[h, t] .} \\
& {[\hat{e}, \hat{t}]=[e, t] a^{2} b^{2} .} \\
& {[\hat{f}, \hat{t}]=[f, t] a^{2} b^{2} .}
\end{aligned}
$$

So the switch to hats gives (1) $\leftrightarrow(2),(3) \leftrightarrow(4)$. 
We will now show (by fusion arguments) that under our hypotheses, the groups $T$ obtained in Cases 2.3.1 and 2.3.3 cannot be Sylow 2-subgroups of a simple group $G$. This will complete the proof of Theorem 2 . We will also show that the $T$ of Case 2.3.1 is isomorphic to the $T$ of Case 1.1 of Theorem 1, and indeed will prove a stronger statement (Theorem 3 ) about this $T$.

Theorem 3 (Proposition 2.3.1). Suppose $T$ is the 2-group obtained in Case 2.3.1 of Theorem 2, namely:

$$
A=\langle a\rangle \times\langle b\rangle \cong Z_{4} \times Z_{4}
$$

$R$ is the split extension of $A$ by a four-group $\{1, e, f, h\}$ where $e, f, h$ have matrices $\left(\begin{array}{ll}3 & 0 \\ 2 & 3\end{array}\right),\left(\begin{array}{ll}3 & 2 \\ 0 & 3\end{array}\right),\left(\begin{array}{ll}1 & 2 \\ 2 & 1\end{array}\right)$ respectively on $A$ (with respect to the basis $\{a, b\}$ of $A$ ).

$$
T=\left\langle R, t: t^{2}=1 ; a^{t}=b, b^{t}=a ;[h, t]=a^{3} b,[e, t]=h b^{3},[f, t]=h a\right\rangle .
$$

If $G$ is a group with $T$ as a Sylow 2-subgroup and $N_{G}(T)=T C_{G}(T)$, then $G$ is not simple. (In particular, this $T$ cannot occur under the hypotheses of Theorem 2.)

Moreover, $T$ is isomorphic to the 2-group obtained in Case 1.1 of Theorem 1. (In particular, if $G$ is a simple group with a Sylow 2-subgroup isomorphic to that of Case 1.1 of Theorem 1, then necessarily $\left[N_{G}(T): C_{G}(T)\right]$ is divisible by some odd prime.)

Proof of Theorem 3. We first list the $T$-classes and the $T$-centralizers of the involutions of $T$, and the same for the elements of order 4 .

Involutions.

$\left\{a^{2} b^{2}\right\}$; central in $T$.

$\left\{a^{2}, b^{2}\right\} ; C_{T}\left(a^{2}\right)=C_{T}\left(b^{2}\right)=R$.

$e W \cup f b W$.

$f W \cup e a W$.

$h W \cup h a b W . C_{T}(e)=C_{T}(f)=C_{T}(h)=\langle e, f, W\rangle \cong E_{16}$.

$t\left\langle a b^{-1}\right\rangle \cup t h a^{2} b\langle a b\rangle ; C_{T}(t)=\langle t\rangle \times\langle h b, a b\rangle \cong Z_{2} \times Q_{8}$.

(te $A$ and $\operatorname{tf} A$ contain no involutions since $T / A \cong D_{8}$ with te $A$ and $t f A$ of order 4.)

Elements of order 4.

$a b W ; C_{T}(a b)=\langle t, A\rangle$ of order $2^{5}$.

$a W \cup b W ; C_{T}(a)=A$ of order $2^{4}$.

$e b W \cup f a b W ; C_{T}(e b)=A_{1}$ (see table for $R / W$ below) of order $2^{4}$.

$f a W \cup e a b W ; C_{T}(f a)=A_{2}$ of order $2^{4}$.

$h a W ; C_{T}(h a)=\left\langle A_{1}, t a b\right\rangle$ of order $2^{5}$.

$h b W ; C_{T}(h b)=\left\langle A_{2}, t\right\rangle$ of order $2^{5}$.

$t b^{2}\left\langle a b^{-1}\right\rangle \cup t h a^{2} b^{-1}\langle a b\rangle ; C_{T}\left(t b^{2}\right)=\left\langle t b^{2}\right\rangle \circ\langle a b, h b\rangle \cong Z_{4} \circ Q_{8}$ of order $2^{4}$.

(te $A$ and $\operatorname{tf} A=(t e A)^{t}$ contain no elements of order 4. For if $x \in A$,

$$
\begin{aligned}
(t e x)^{2} & =[t, e] x^{t e} x=\left(h b^{3}\right)^{-1} x^{t e} x \\
& =b h x^{t e} x=h a^{2} b x^{t e} x .
\end{aligned}
$$


Now for all $x \in A$,

$$
x^{t e} x \in\left\langle a^{t e} a, b^{t e} b\right\rangle \subseteq\langle a b, W\rangle .
$$

So $h a^{2} b x^{t e} x$ is always of order 4 since it never lies in $h W \cup h a b W$.

The group $R / W$ is as follows:

\begin{tabular}{|c|c|c|c|c|}
\hline$e a b W$ & $e b W$ & $a W$ & $e W$ & $e a W$ \\
\hline$f a W$ & $f a b W$ & $b W$ & $f W$ & $f b W$ \\
\hline$h b W$ & haW & $a b W$ & $h W$ & $h a b W$ \\
\hline$A_{2}$ & $A_{1}$ & $A$ & $E$ & $E^{t}$ \\
\hline
\end{tabular}

The vertical columns are four-groups in $R / W$, whose inverse images in $R$ we give the names $A_{1}, A_{2}, A, E, E^{t}$ as shown. $A_{1}, A_{2}, A \cong Z_{4} \times Z_{4}$ and are normal in $T$; $E, E^{t} \cong E_{16}$.

$R$ contains three $Q_{8} \times Z_{2}$ 's, namely the inverse images of the horizontal boxes. $R$ normalizes them, and in each one it acts transitively on the four $Q_{8}$ 's; i.e., each element $x$ of order 4 is sheared onto each central involution $w$ (i.e., there is $y$ with $x^{y}=x w$, or equivalently, $\left.[x, y]=w\right)$.

Suppose $G$ is a simple group with $T$ as a Sylow 2-subgroup and $N_{G}(T)=T C_{G}(T)$. By Lemma A, $t$ is fused into $R$ in $G$. If $t$ is not fused to $W$, then $C_{T}(t)$ is a Sylow 2-subgroup of $C_{G}(t)$, and also some conjugate of the $T$-centralizer of an involution of $R-A$ is a Sylow 2-subgroup of $C_{G}(t)$. But $C_{T}(t) \cong Z_{2} \times Q_{8}$ and the $T$-centralizers of the involutions of $R-A$ are $\cong E_{16}$, contradicting Sylow's theorem in $C_{G}(t)$.

So $t$ is fused to $W$ in $G$.

There is $d \in G$ with $t^{d} \in W$ and $C_{T}(t)^{d} \subseteq C_{T}\left(t^{d}\right)$. For if $t$ is fused to $a^{2} b^{2}$, take $d \in G$ with $t^{d}=a^{2} b^{2} ; T$ is then a Sylow 2-subgroup of $C_{G}\left(t^{d}\right)$. If $t$ is not fused to $a^{2} b^{2}$, take $d \in G$ with $t^{d}=a^{2}$ (or $b^{2}$ ); $R$ is then a Sylow 2-subgroup of $C_{G}\left(t^{d}\right)$. In either case, Sylow's theorem (in $C_{G}\left(t^{d}\right)$ ) implies that $d$ can be adjusted by multiplication from $\boldsymbol{C}_{G}\left(t^{d}\right)$ to have the property claimed.

Now $C_{T}(t)=\langle t\rangle \times\langle a b, h b\rangle$ has $\left\langle t, a^{2} b^{2}\right\rangle$ as its central four-group, and $a^{2} b^{2}$ as its square. The only involutions of $T$ which are squares of $Q_{8}$ 's of $T$ are $a^{2}, b^{2}$, and $a^{2} b^{2}$. So $\left(a^{2} b^{2}\right)^{d}$ and $t^{d}$ are both $\in W$; therefore $Z\left(C_{T}(t)^{d}\right)=W$. This forces $C_{T}(t)^{d}$ to be one of the three $Q_{8} \times Z_{2}$ 's of $R$.

Therefore in $N_{G}\left(C_{T}(t)^{d}\right)$, each element of order 4 is sheared onto $t$. In particular, $h b$ is conjugate to $t h b$ and $h a b^{2}$ to thab $b^{2}$. Now $t h b$ and $t h a b^{2}$ are $T$-conjugate, as are $h a b^{2}$ and $h a$; so $h b$ is $G$-conjugate to $h a$.

We now observe that there can be no fusion in $G$ between $h b, h a$, and $a b$ (thus we have reached a contradiction and so proved Theorem 3), For the centralizers 
in $T$ of $h b, h a$, and $a b$ are Sylow 2-subgroups of their centralizers in $G$. If (for example) $h b$ is fused to $h a$, there is $d \in G$ with $(h b)^{d}=h a$ and $C_{T}(h b)^{d}=C_{T}(h a)$. $C_{T}(h b), C_{T}(h a)$ contain $A_{2}, A_{1}$ as characteristic subgroups (e.g., because these are the only Abelian subgroups of index 2), so $A_{2}^{d}=A_{1}$. Therefore $N_{G}\left(A_{2}\right)^{d}=N_{G}\left(A_{2}^{d}\right)$ $=N_{G}\left(A_{1}\right) . T^{d}$ and $T$ are both Sylow 2-subgroups of $N_{G}\left(A_{1}\right)$ so there is $k \in N_{G}\left(A_{1}\right)$ with $A_{2}^{d k}=A_{1}$ and $T^{d k}=T$, i.e. $d k \in N_{G}(T)$. But $N_{G}(T)=T C_{G}(T)$, and $A_{2}$ and $A_{1}$ are not conjugate in $T C_{G}(T)$.

We now show that $T$ is isomorphic to the 2-group $U$ say obtained in Case 1.1 of Theorem 1. $U$ was given by:

$$
\begin{gathered}
W=\left\langle z_{1}, z_{2}\right\rangle . \\
C_{U}(W)=\left\langle W, i, j, k, x, u, v:\langle i, j, k\rangle \cong Q_{8} \text { with } z_{1}\right. \text { as square, and } \\
\langle x, u, v\rangle \cong Q_{8} \text { with } z_{1} z_{2} \text { as square; } \\
{[i, x]=1,[j, x]=z_{2},[k, x]=z_{2}} \\
{[i, u]=z_{2},[j, u]=1,[k, u]=z_{2}} \\
\left.[i, v]=z_{2},[j, v]=z_{2},[k, v]=1 .\right\rangle
\end{gathered}
$$

(We write $x$ instead of the $t$ used in Theorem 1, to avoid confusion with the $t$ of Case 2.3.1 of Theorem 2.)

$$
\begin{aligned}
U=\left\langle C_{U}(W), \tau: \tau^{t}=1 ; z_{1}^{\tau}=z_{1},\right. & z_{2}^{\tau}=z_{1} z_{2} ; \\
& \left.\begin{array}{r}
\tau \text { centralizes } i, j, \text { and } k ; \\
{[x, \tau]}
\end{array}=i z_{2},[u, \tau]=j z_{2},[v, \tau]=k z_{2}\right\rangle .
\end{aligned}
$$

We first show $R \cong C_{U}(W)$. For take $i, j, k=a b, h b, h a b^{2}$ respectively; $z_{1}=a^{2} b^{2}$. $x, u, v=a, e a b, e b$ respectively; $z_{1} z_{2}=a^{2}$ (so that $z_{2}=b^{2}$ ). Then the relations above for $z_{1}, z_{2}, i, j, k, x, u, v$ are satisfied.

Now once we find a set $\left\{z_{1}, z_{2}, i, j, k, x, u, v\right\}$ satisfying these relations, the set $\left\{z_{1}, z_{2}, i, j, k, x^{-1}, u^{-1}, v^{-1}\right\}$ also satisfies these relations (since $W$ is elementary and central in $C_{U}(W)$ ). Noting this, we take $\tau=t$. $t$ centralizes $i, j$, and $k$, and acts as prescribed on $W$. And

i.e.,

$$
\begin{aligned}
{\left[a^{-1}, t\right] } & =(a b) b^{2}, \\
{\left[(e a b)^{-1}, t\right] } & =(h b) b^{2}, \\
{\left[(e b)^{-1}, t\right] } & =\left(h a b^{2}\right) b^{2},
\end{aligned}
$$

$$
\begin{aligned}
& {\left[x^{-1}, \tau\right]=i z_{2}} \\
& {\left[u^{-1}, \tau\right]=j z_{2}} \\
& {\left[v^{-1}, \tau\right]=k z_{2}}
\end{aligned}
$$

Thus, the generators $z_{1}=a^{2} b^{2}, z_{2}=b^{2} ; i, j, k=a b, h b, h a b^{2} ; x, u, v=a^{-1},(e a b)^{-1}$, $(e b)^{-1} ; \tau=t$ satisfy precisely the relations given for $U$. 
At this point it is easy to see that $T$ is a split extension of a $Q_{8} \circ D_{8}$ by a fourgroup (which is the way in which the Sylow 2-subgroup of the Janko-Hall and Janko-Higman groups is usually described). For, using the notation for $T$ obtained in Theorem 2, $Q=\left\langle a b, h b, h a b^{2}\right\rangle \cong Q_{8}$ with $a^{2} b^{2}$ as square; $D=\langle t, W\rangle \cong D_{8}$; and $[D, Q]=1, D \cap Q=\left\langle a^{2} b^{2}\right\rangle$, so $D Q=D \circ Q=H$ say. It is convenient to depict $H$ by making a table consisting of a representative for each coset of $\left\langle a^{2} b^{2}\right\rangle$ in $H$, as follows:

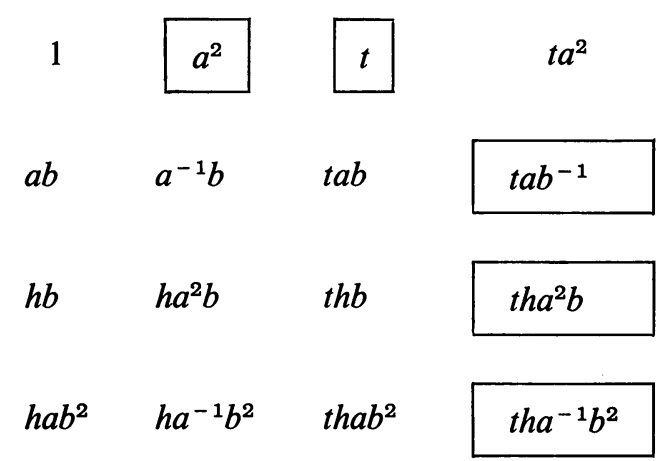

where the cosets consisting of involutions are the ones in boxes. These cosets generate $H$.

$\langle e, f\rangle \cap H=1$; and $\langle e, f\rangle$ acts on the set $\left\{t, t a b^{-1}, t h a^{2} b, t h a^{-1} b^{2}\right\}$ as follows:

$$
\begin{gathered}
h: t \leftrightarrow t a b^{-1}, t h a^{2} b \leftrightarrow t h a^{-1} b^{2} \\
e: t \leftrightarrow t h a^{2} b, t a b^{-1} \leftrightarrow t h a^{-1} b^{2} \\
f: t \leftrightarrow t h a^{-1} b^{2}, t a b^{-1} \leftrightarrow t h a^{2} b .
\end{gathered}
$$

Thus $\langle e, f\rangle$ normalizes $H$, and $T$ is as claimed.

Proposition 2.3.3. Suppose T is the 2-group obtained in Case 2.3.3 of Theorem 2, namely:

$A=\langle a\rangle \times\langle b\rangle \cong \boldsymbol{Z}_{\mathbf{4}} \times \boldsymbol{Z}_{\mathbf{4}}$.

$R=\left\langle A, e, f, h: e, f, h\right.$ have matrices $\left(\begin{array}{ll}3 & 0 \\ 2 & 3\end{array}\right),\left(\begin{array}{ll}3 & 2 \\ 0 & 3\end{array}\right),\left(\begin{array}{ll}1 & 2 \\ 2 & 1\end{array}\right)$ respectively on $A$ (with respect to the basis $\{a, b\}$ of $A) ; e^{2}=b^{2}, f^{2}=a^{2} b^{2}, h^{2}=a^{2} ; e f a^{2}=h ; e, f, h$ centralize one another $>$.

$T$ is the split extension of $R$ by a cyclic group $\langle t\rangle$ of order 4 , where $t^{2}=z$ inverts $A$, and $t$ has the matrix $\left(\begin{array}{ll}2 & 3 \\ 1 & 2\end{array}\right)$ on $A$;

$$
\begin{array}{lll}
{[e, t]=h a^{3} b^{3},} & {[f, t]=h b,} & {[h, t]=a^{3} ;} \\
{[e, z]=a^{-1},} & {[f, z]=b^{-1},} & {[h, z]=a b .}
\end{array}
$$

Suppose $T$ is a Sylow 2-subgroup of a group $G$, and suppose the involutions of $W=\Omega_{1}(A)$ are all fused together in $G$. Then $G$ is not simple.

In particular, this $T$ cannot occur under the hypotheses of Theorem 2. 
Proof of Proposition 2.3.3.

(xvii) The only cosets of $A$ in $T$ which contain involutions are $A$ and $z A$.

Proof. $T / R$ is cyclic, so the involutions of $T$ lie in $T_{0}=\langle z, R\rangle$. The only involutions of $R$ are those of $A$; and the only involutions of $z R$ are those of $z A$, since for $x \in A$ and $k=e, f$, or $h$,

$$
\begin{aligned}
(z k x)^{2}=(z k)^{2} x^{z k} x & \equiv[z, k] x^{z k} x \bmod W \\
& \equiv[z, k] \bmod W
\end{aligned}
$$

but $[z, k] \notin W$.

(xviii) The $T$-classes of the elements of order 4 in $T$, and the orders of the $T$ centralizers of representatives, are:

Class

1. $a b W$.

2. $a W \cup b W$.

3. $e W \cup f a b W \cup e a W \cup f a W$.

4. Complement of 3. in $e A \cup f A$.

5. $h A$.

6. $t A \cup \operatorname{th} A$.

7. $t z A \cup \operatorname{tzh} A$.
Order of $T$-centralizer of a representative

Proof. It helps to note that if $x \in A$ and $k=1, e, f$, or $h$,

$$
\begin{aligned}
(t k x)^{2} & =(t k)^{2} x^{t k} x=t^{2} k^{t} k x^{t k} x \\
& =z k^{t} k x^{t k} x,
\end{aligned}
$$

which is an involution if and only if $k^{t} k \in A$ (by (xvii)). This occurs for $k=1$ and $k=h$. The same holds if $t$ is replaced by $t z=t^{-1}$.

$h$ and $A$ conjugate together the elements of $t A ;(t A)^{e}=t h A$.

(xix) The only $Z_{4} \times Z_{4}$ 's of $T$ lie in $R$.

Proof. The only elements of order 4 with centralizers of order $\geqq 16$, lie in $R$ by (xviii).

(xx) For $y \in A, y$ of order 4, the only involutions of $C_{T}(y)$ are those of $W$.

Proof. $C_{T}(y)$ is a union of cosets of $A$, but no element of $z A$ centralizes $y$; statement follows from (xvii).

(xxi) If $G$ is simple then $t$ is fused into $A$.

Proof. Consider the transfer homomorphism $\tau: G \rightarrow T / R$. Its value at $z$ is

$$
\tau(z)=\prod\left\{y z y^{-1}: \text { cosets } T y \text { with } T y z=T y\right\} R .
$$

If no such $y z y^{-1}$ lay in $R$, they would all lie in $z R$. Since the number of cosets $T y$ with $T y z==T y$ is odd, we would have $\tau(z) \neq 1$, contradicting simplicity of $G$.

Therefore $z$ is fused into $R$ in $G$. Hence there is $d \in G$ with $z^{d}=a^{2} b^{2}$ and $C_{T}(z)^{d} \subseteq T$. The elements of $T-A$ whose squares are $a^{2} b^{2}$ are $e a^{\xi} b^{\eta}$ for $\eta$ odd, and $f a^{\xi} b^{\eta}$ for 
$\xi$ even. These are all $T$-conjugate by (xviii). So if $t^{d} \in C_{T}(z)^{d}$ is not in $A$, we may assume $t^{d}=e b$.

$C_{T}(z)=\left(\langle t\rangle \times\left\langle a^{2} b^{2}\right\rangle\right)\left\langle a^{2}\right\rangle$ where $a^{2}$ shears $t$ onto $a^{2} b^{2}$ (i.e., $t^{a^{2}}=t a^{2} b^{2}$, i.e., $\left.\left[t, a^{2}\right]=a^{2} b^{2}\right)$. So in $C_{T}(z)^{d} \subseteq T$ with $t^{d}=e b$, there must be involutions $x, y$ such that $x$ centralizes $e b$ and $x \neq(e b)^{2}=a^{2} b^{2}$, and $y$ shears $e b$ onto $x$ and $y \notin\langle e b, x\rangle$. $x, y \in A \cup z A$ by (xvii).

But for $c \in A$,

$$
\begin{aligned}
{[e b, z c] } & =[e b, c][e b, z]^{c} \\
& =[e, c][e, z][b, z] \equiv[e, z] \bmod W
\end{aligned}
$$

and $[e, z] \notin W$.

So no element of $z A$ centralizes $e b$. So $x \in A$, and $\langle e b, x\rangle \supseteq W$. But the same calculation shows that no element of $z A$ can shear $e b$ onto $x \in W$. So $y \in A$, hence $\in W \subseteq\langle e b, x\rangle$; thus there is no suitable $y \in T$.

This contradiction establishes that $t^{d} \in A$, as desired.

Now suppose that $G$ is simple. By (xxi), there is $d \in G$ with $t^{d} \in A$. Write

$$
V=C_{T}\left(t^{d}\right)^{d^{-1}}, \quad V_{0}=A^{d^{-1}}
$$

Then $V \supseteq V_{0}$ and $V_{0} \cong Z_{4} \times Z_{4}$.

Take $t^{d}=a b$ if possible; otherwise, take $t^{d}=a$ or $b$. Then by (xviii), $V$ is a Sylow 2-subgroup of $\boldsymbol{C}_{T}(t)$.

Let $U$ be a conjugate of $V$ by an element of $C_{G}(t)$ such that $U \supseteq C_{T}(t)$ $=\langle t\rangle \times\left\langle a^{2} b^{2}\right\rangle=F$ say; let $U_{0}$ be the corresponding conjugate of $V_{0} . t \in U_{0}$, and also $a^{2} b^{2} \in \Omega_{1}\left(U_{0}\right)$ by (xx). Therefore $F \subseteq U_{0}$; and $U_{0}$ normalizes $F$.

$N_{T}(F)=\left\langle F, a^{2}\right\rangle$, where $a^{2}$ shears $t$ onto $a^{2} b^{2}$ (i.e., $\left[t, a^{2}\right]=a^{2} b^{2}$ ).

Let $U_{2} \supseteq U_{0}$ be a Sylow 2-subgroup of $N_{G}(F)$, and let $U_{3} \supseteq U_{2}$ be a Sylow 2subgroup of $G$.

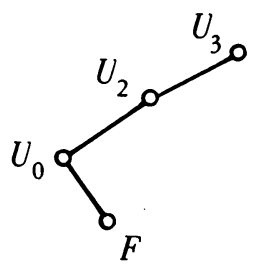

Let $T^{*}$ be a suitable conjugate of $U_{3}$ by an element of $N_{G}(F)$ so that the corresponding conjugate $U_{2}^{*}$ contains $N_{T}(F)$. Thus in $T^{*}, t$ lies in a $Z_{4} \times Z_{4}$ (namely, the corresponding conjugate $U_{0}^{*}$ of $U_{0}$ ) which also contains $F=\left\langle t, a^{2} b^{2}\right\rangle$ with index 2; and there is an involution (namely $a^{2}$ ) in $T^{*}-\Omega_{1}(F)=T^{*}-W^{*}$ which shears $t$ onto $a^{2} b^{2}$. $\left(\Omega_{1}(F)=\Omega_{1}\left(U_{0}^{*}\right)=W^{*}\right.$; for $U_{0}^{*}$ is a $Z_{4} \times Z_{4}$ of $T^{*}$, so $U_{0}^{*} \subseteq R^{*}$ by (xix), hence $\Omega_{1}\left(U_{0}^{*}\right)=W^{*}$.)

We now observe that no element of order 4 in $T^{*}$ which lies in a $Z_{4} \times Z_{4}$ ( $Y^{*}$ say) of $T^{*}$, can be sheared by an involution of $T^{*}-W^{*}$ onto an independent involution 
$\in Y^{*}$. (By Sylow's theorem, this is equivalent to the same statement with the stars removed.)

For by (xix), $Y \subseteq R$ and so its elements of order 4 are of the form $k x$ for $x \in A$ and $k=1, e, f$, or $h$. The only involutions of $T-W$ are $z c$ for $c \in A$, by (xvii).

$$
\begin{aligned}
{[k x, z c] } & =[k x, c][k x, z]^{c} \quad \text { but }[k x, z] \in A, \text { so commutes with } c \\
& =[k, c][k, z][x, z]=[k, c][k, z] x^{2} .
\end{aligned}
$$

If $k$ is $e, f$, or $h$, then $[k, c]$ and $x^{2} \in W$ but $[k, z] \notin W$, so the commutator $[k x, z c]$ is not an involution. If $k=1$, we have $[x, z c]=x^{2}$ and so our element $x$ of order 4 is inverted by the involution $z c$-not sheared onto an independent involution.

This contradiction completes the proof of Proposition 2.3.3.

\section{REFERENCES}

1. J. Alperin, Centralizers of Abelian normal subgroups of p-groups, J. Algebra 1 (1964), 110-113. MR $29 \# 4800$.

2. N. Blackburn, On a special class of p-groups, Acta Math. 100 (1958), 45-92. MR 21 \#1349.

3. - Generalizations of certain elementary theorems on p-groups, Proc. London Math. Soc. (3) 11 (1961), 1-22. MR 23 \#A208.

4. R. Brauer, Some applications of the theory of blocks of characters of finite groups. II, J. Algebra 1 (1964), 307-334. MR 30 \#4836.

5. W. Feit, Characters of finite groups, Notes printed by Yale University, New Haven, Conn., 1965.

6. W. Feit and J. Thompson, Solvability of groups of odd order, Pacific J. Math. 13 (1963), 775-1029. MR 29 \#3538.

7. G. Glauberman, Central elements in core-free groups, J. Algebra 4 (1966), 403-420. MR 34 \#2681.

8. G. Higman, Suzuki 2-groups, Illinois J. Math. 7 (1963), 79-96. MR 26 \#1365.

9. B. Huppert, Endliche Gruppen. I, Die Grundlehren der math. Wissenschaften, Band 134, Springer-Verlag, Berlin and New York, 1967. MR 37 \#302.

10. J. Thompson, Non-solvable finite groups whose nonidentity solvable subgroups have solvable normalizers (to appear).

UNIVERSity OF ChICAGo,

Chicago, Illinois 60637 